$$
\begin{aligned}
& \text { PRE } 34059 \\
& \text { SCU } 9405
\end{aligned}
$$

\title{
MASS GENERATION IN THE LARGE N GROSS-NEVEU-MODEL
}

\author{
C. KOPPER \\ Institut für Theoretische Physik der Universität Göttingen \\ Bunsenstrasse 9 - D-37073 Göttingen \\ J. MAGNEN and V. RIVASSEAU \\ Centre de Physique Théorique, CNRS, UPR 14 \\ Ecole Polytechnique - 91128 Palaiseau Cedex.
}

\begin{abstract}
We study the infrared behaviour of the Euclidean Gross-Neveu-Model with discrete chiral symmetry. Imposing a suitable UV-cutoff we prove that for a large (but finite !) number of fermion components the model has (at least) two pure phases, realized by suitable boundary conditions and that the fermion two-point function decays exponentially.
\end{abstract}




\section{INTRODUCTION}

We want to study the infrared behaviour of the two-dimensional Euclidean Gross-Neveu model [1] which is formally given through the Lagrangian

$$
\mathscr{Z}=\bar{\psi}(\mathrm{x}) \mathrm{i} \not \partial \psi(\mathrm{x})-\frac{\lambda}{2 \mathrm{~N}}(\bar{\psi}(\mathrm{x}) \psi(\mathrm{x}))^{2}
$$

for $\mathrm{N} \gg$ 1. Here $\mathrm{N}$ is the number of fermion flavours, i.e.

$$
\psi=\left(\psi_{1}, \ldots, \psi_{\mathrm{N}}\right)^{\mathrm{T}},\left(\bar{\psi}=\bar{\psi}_{1}, \ldots, \bar{\psi}_{\mathrm{N}}\right)
$$

The coupling $\lambda$ is supposed to be a constant of order 1 . Since we will study the model with an UVcutoff, it does not make much sense to choose $\lambda \geq \pi$ : we will show that the model is massive and that the mass approaches the size of the cutoff for large $\lambda>\pi$. If $\lambda \ll<\pi$ there arises a technical difficulty : the mass decreases as $\mathrm{e}^{-\pi / \lambda}$, and the correlations decay more and more slowly. But the cluster expansions can only be shown to converge for $\mathrm{N} \gg \mathrm{m}^{-1}$. So small $\lambda$ requires (very) large N.

Our aim is to show that the mechanism of mass generation discovered by Gross and Neveu and analyzed by them to first order in $1 / \mathrm{N}$ persists in the full model for $\mathrm{N}$ sufficiently large. Being mainly interested in the IR behaviour of the model we will therefore study the model with an UV cutoff, the scale of which is put equal to one.

The UV limit will be postponed to another paper, and one should note in this respect that the UV limit of the two-dimensional massive (by hand !) [2,3] and of the three-dimensional large $\mathrm{N}$ [4] Four-Fermion-Models have already been constructed.

In our study we will present the model in terms of an auxiliary bosonic field $\sigma$. Its action is obtained by formally integrating out the fermionic fields $\bar{\psi}, \psi$. This representation while providing the same perturbative expansion as the original fermionic one is better suited to the large $\mathrm{N}$ behaviour of the model. The thermodynamic limit and the Green functions of the model will be controlled in this paper with appropriate cluster expansion techniques and bounds to estimate its (nonlocal) interaction. Our main interest is to show that the model is massive, i.e. that the two-point function falls off exponentially

$$
\left|S_{2}(x, y)\right| \leq K e^{-m^{\prime}|x-y|}
$$


with some (in principle calculable) $\mathrm{m}^{\prime}>0$ (see (209)), and that it has two phases at 0 temperature ${ }^{1}$ which can be realized by imposing suitable boundary conditions before taking the thermodynamic limit. In the language of the $\sigma$ field these 2 phases correspond to 2 opposite magnetizations (nonzero v.e.v. for the $\sigma$ field). So the situation turns out to be quite analogous to that of the asymmetric $\varphi^{4}$ theory with an interaction $\lambda\left(\varphi^{2}-\lambda^{-1}\right)^{2}, \lambda \ll 1$ presented in [5], ch. 16.

As long as we don't take the UV limit the additional difficulties are mainly stemming from two facts :

1) Our model is massless in the beginning and the mass is physically present only for $\sigma$ field values close to the minima of the action (depending in sign on which vacuum state is chosen) so that the treatment and the expansions depend on the "size" of the $\sigma$-field. If $\sigma$ is close to one of the minima a local translation of the field variable $\sigma$ will be performed.

2) The interaction of the model is non-polynomial and non-local in the $\sigma$-field. The analogy with $\lambda\left(\phi^{2}-\lambda^{-1}\right)^{2}$ implies that the model is indeed qualitatively well represented for large $\mathrm{N}$ by the "effective potential" $V(\sigma)$ which has been calculated by Gross and Neveu [1] (by effective potential we mean the value of the $\sigma$-Lagrangian as a function of $\sigma$ for constant $\sigma$ ), see Fig. 1 .

\section{PRESENTATION OF THE MODEL}

The aim of the paper is to show the existence and to derive bounds for the fermionic twopoint function $\langle\bar{\psi}(\mathrm{x}) \psi(\mathrm{y})>$. The treatment of $\mathrm{N}$ point functions will be in obvious analogy. Our heuristic starting point is thus

$$
\mathrm{S}_{2}(\mathrm{x}, \mathrm{y}) \sim \int \mathrm{D}(\psi, \bar{\psi}) \bar{\psi}_{\mathrm{i}}(\mathrm{x}) \psi_{\mathrm{i}}(\mathrm{y}) \mathrm{e}^{-\int\left(\bar{\psi} p \psi-1 / 2 \mathrm{~g}^{2}(\bar{\psi} \psi)^{2}\right)}
$$

where

$\mathrm{g}^{2}=\frac{\lambda}{\mathrm{N}}, \not p=\mathrm{p}_{0} \gamma_{0}+\mathrm{p}_{1} \gamma_{1} ; \gamma_{0}, \gamma_{1}$ are the two dimensional Euclidean $\gamma$ matrices with

$$
\left\{\gamma_{\mu}, \gamma_{v}\right\}=-2 \delta_{\mu \nu}, \gamma_{\mu}^{+}=-\gamma_{\mu}, \text { e.g. }
$$

\footnotetext{
1 Strictly speaking we prove the existence of at least two phases. More than two phases can presumably be ruled out as in Ising models by means of correlation inequalities.
} 
(2)

$$
\gamma_{0}=\left(\begin{array}{cc}
0 & \mathrm{i} \\
\mathrm{i} & 0
\end{array}\right) \quad \gamma_{1}=\left(\begin{array}{cc}
\mathrm{i} & 0 \\
0 & -\mathrm{i}
\end{array}\right)
$$

On introducing formally the $\sigma$ field through

$$
S_{2}(\mathrm{x}, \mathrm{y}) \sim \int \mathrm{D}(\psi, \bar{\psi}) \mathrm{D} \sigma \bar{\psi}_{\mathrm{i}}(\mathrm{x}) \psi_{\mathrm{i}}(\mathrm{y}) \exp \left\{-\int\left(\bar{\psi} p \psi+1 / 2 \sigma^{2}+\mathrm{g} \sigma \bar{\psi} \psi\right)\right\}
$$

we may integrate out the fermions arriving at

$$
S_{2}(x, y) \sim \int D \sigma\left(\frac{1}{\not p+g \sigma}\right)_{i}(x, y) \operatorname{det}(\not p+g \sigma) e^{-1 / 2 j \sigma^{2}}
$$

where $\left(\frac{1}{p+g \sigma}\right)_{i}(x, y)$ is the position space kernel of $\frac{1}{p+g \sigma}$ sandwiched between projectors on the $i$-th flavour subspace. Expression (4) is still highly formal since the infinite -dimensional Lebesgue measure $\mathrm{D} \sigma$ is ill-defined and since the terms written are plagued with infrared (IR) and ultraviolet (UV) divergences. We therefore introduce the following regularizations :

\section{UVI :}

Let $\mathrm{u}\left(\mathrm{p}^{2}\right)$ be a smooth nonnegative function with $\mathrm{u}(0)=1,1 / \mathrm{u} \in \mathfrak{L}^{2}\left(\mathbb{R}^{2}\right)$ (viewed as a function of $\mathrm{p}$ ). To be definite we set

$$
u(p)=e^{1 / 2 p^{2}}
$$

$$
p_{\mathrm{rg}}=p_{\mathrm{u}}=p \mathrm{pu}\left(\mathrm{p}^{2}\right) \text { (and analogously for functions of } p \text { ) }
$$

$\mathrm{u}$ will thus regularize the fermion propagator. We have not explicitly introduced a cutoff scale in the definition of $\mathrm{u}$ which means that the cutoff scale is chosen equal to one.

Contrary to perturbation theory where the regularization of the fermion propagator suffices to regularize all diagrams since any $\sigma$-propagator is sandwiched between fermions we also need a regularization of the $\sigma$-field here. Otherwise we would encounter (at least) considerable technical complications, in particular we could not prove Lemma 3 below.

The $\sigma$-field appears as an ultralocal field in (3), (4), but the $\sigma$-cutoff will be a new source of 
nonlocality which in turn leads to difficulties when the translation of the $\sigma$ variable is performed. To minimize those we impose some conditions on the cutoff.

UVII : The regularizing function will be called $\hat{f}_{\rho}(p)$ and will depend on $\mathrm{p}^{2}$ only. We write it in the form

$$
\hat{\mathrm{f}}_{\rho}=\mathrm{af}_{\rho} \mathrm{a} \quad \rho \geq 1
$$

where $\mathrm{a}\left(\mathrm{p}^{2}\right)$ is a smooth bounded strictly positive function of $\mathrm{p}^{2}$ which will be specified later $\left(\mathrm{a}\left(\mathrm{p}^{2}\right)=\sqrt{\mu^{2}+\pi_{\text {ren }}}\right.$, see $\left.(83)\right)$

$$
0<\mu \leq \mathrm{a}\left(\mathrm{p}^{2}\right) \leq \mathrm{O}(1)
$$

We first define $f_{1}$. Apart from Euclidean symmetry we demand

i) $f_{1} \in C^{\infty}\left(\mathbb{R}^{2}\right)$

ii) $\mathrm{f}_{1}(\mathrm{p}) \geq 0 ; \mathrm{f}_{1}(0)=0 ; \mathrm{f}_{1}(\mathrm{p}) \leq \mathrm{A}|\mathrm{p}|^{2 \mathrm{~L}},|\mathrm{p}|<1 ; \alpha\left(\mathrm{p}^{2}\right)^{2}<\mathrm{f}_{1}(\mathrm{p})<\mathrm{A}\left(\mathrm{p}^{2}\right)^{2},|\mathrm{p}|>1$.

ii) With $g(p)=\frac{1}{1+f_{1}(p)}$ we also demand that $\tilde{g}(x)=\int g(p) e^{i p x} \frac{d^{2} p}{(2 \pi)^{2}}$ has compact support, i.e.

$\widetilde{\mathrm{g}}(\mathrm{x}) \equiv 0$ for $|\mathrm{x}| \geq C$.

Here $\mathrm{C}, \alpha, \mathrm{A}>\alpha$ are suitable positive constants. They may be chosen (arbitrarily) small. $\mathrm{L} \in \mathbb{N}$ has to be fixed for later purposes not too small, to be definite we set

$$
\mathrm{L}=32
$$

\section{Lemma 1:}

(i) There exist functions $\mathrm{f}_{1}$ fulfilling (9)

(ii) If $\mathrm{f}_{1}$ fulfills $(9)$, then, for $\rho>0, \mathrm{f}_{\rho}(\mathrm{p}):=\mathrm{f}_{1}\left(\frac{\mathrm{p}}{\rho}\right)$ fulfills $(9)$ on replacing $|\mathrm{p}| \gtrless 1$ by $|\mathrm{p}| \gtrless \rho$ in $(9$ ii) and $\mathrm{C}$ by $\mathrm{C} / \mathrm{\rho}$ in ( 9 iii).

Remark: We will fix $\rho>1$ as a function of $\mathrm{N}$ for some given $\mathrm{f}$ later on (see (61)). 
Proof: (i) We start from $\widetilde{\mathrm{G}}(\mathrm{x})= \begin{cases}1 & |\mathrm{x}|<1 \\ 0 & |\mathrm{x}| \geq 1\end{cases}$

Its Fourier transform is $G(p)=\int d^{2} x e^{-i p x} \widetilde{G}(x)=2 \pi \int_{0}^{1} x d x J_{0}(|p| x)$, where the Bessel function $\mathrm{J}_{0}(\mathrm{z})$ is bounded by const $\cdot \mathrm{z}^{-1 / 2}$ so that

$$
|\mathrm{G}(\mathrm{p})| \leq \mathrm{C} \frac{1}{1+|\mathrm{p}|^{1 / 2}}
$$

Note that $G$ depends on $\mathrm{p}^{2}$ only. Choose an even integer $M \geq 4 L+20$. We have

$$
0 \leq \mathrm{G}^{\mathrm{M}}(\mathrm{p}) \leq \mathrm{C}^{\mathrm{M}} \frac{1}{1+|\mathrm{p}|^{\mathrm{M} / 2}},
$$

and the Fourier transform of $\mathrm{G}^{\mathrm{M}}$ is in $\mathrm{C}^{\mathrm{M} / 2-3}\left(\mathbb{R}^{2}\right)$ and supported in $|\mathrm{x}|<\mathrm{M}$.

$\mathrm{G}^{\mathrm{M}}(\mathrm{p})$ is analytic in $\mathrm{p}$ and may be expanded around 0 as

$$
\mathrm{G}^{\mathrm{M}}(\mathrm{p})=\sum_{\mathrm{n}=0}^{\infty} \mathrm{a}_{\mathrm{n}}\left(\mathrm{p}^{2}\right)^{\mathrm{n}}
$$

where $a_{0}=\int\left(\widetilde{G^{M}}\right)(x) d^{2} x>0$, and all coefficients are real.

We now set

$$
G_{1}(p)=\frac{1}{a_{0}}\left(\sum_{n=0}^{L} b_{n}\left(p^{2}\right)^{n}\right) G^{M}(p)
$$

where $b_{0}=1$, and $b_{n}, 1 \leq n<L$ are inductively fixed such that $G_{1}(p)$ has vanishing derivatives up to order $2 \mathrm{~L}$ at zero $\left(b_{1}=-a_{1} / a_{0}, b_{2}=-1 / a_{0}\left(a_{2}+b_{1} a_{1}\right), \ldots\right) ; b_{L}$ is chosen sufficiently small (possibly $<0$ ) as follows : $G_{1}(p)$ may be Taylor expanded at 0 as

$$
\mathrm{G}_{1}(\mathrm{p})=1+\sum_{\mathrm{n}=\mathrm{L}}^{\infty} \mathrm{c}_{\mathrm{n}}\left(\mathrm{p}^{2}\right)^{\mathrm{n}}
$$

and we choose $b_{\mathrm{L}}$ such that $c_{\mathrm{L}}<0$ and so small that

$$
\mathrm{G}_{1}(0)>\mathrm{G}_{1}(\mathrm{p}) \forall \mathrm{p} \neq 0
$$


This is possible, since $G_{1}$ has a local maximum at 0 and by decreasing $b_{L}$ it decreases strictly for any finite $\mathrm{p}$ (note $\mathrm{G}^{\mathrm{M}} \geq 0$ ).

The next step is to build $\mathrm{G}_{2}(\mathrm{p})$ with

$$
\mathrm{G}_{2}(0)>\left|\mathrm{G}_{2}(\mathrm{p})\right| \quad, \quad \mathrm{p} \neq 0
$$

If (11) is fulfilled for $G_{1}$ with suitably chosen $b_{L}$, we set $G_{1}=G_{2}$. If it is not fulfilled for any $b_{L}$, we assume $b_{L}$ has been chosen so small that

$$
\mathrm{G}_{1}>0 \text { for }|\mathrm{p}|<\mathrm{p}_{0}, \mathrm{G}_{1} \leq 0 \text { for }|\mathrm{p}| \geq \mathrm{p}_{0} \text { with some } \mathrm{p}_{0}>0
$$

Then (11) is violated for those $\mathrm{p}$ for which

$$
G_{1}(p) \leq-1
$$

Since

$$
\left|G_{1}\right| \leq \frac{C^{\prime}}{1+|p|^{10}}
$$

this will happen only in some compact region. Set

$$
d=-\inf _{p} G_{1}(p)
$$

We have $1 \leq \mathrm{d}<\infty$. Put

$$
\mathrm{G}_{1}^{(1)}=\mathrm{G}_{1}+\frac{1}{\mathrm{~d}} \mathrm{G}_{1}^{2}
$$

Then

$$
d_{1}=-\inf _{p} G_{1}^{(1)}(p)=\frac{d}{4}
$$

and still

$$
\begin{aligned}
& G_{1}^{(1)}(0)>G_{1}^{(1)}(p), p \neq 0 \\
& G_{1}^{(1)}>0 \text { for }|p|<p_{0}, G_{1}^{(1)} \leq 0 \text { for }|p| \geq p_{0} .
\end{aligned}
$$


If $G_{1}^{(1)}$ does not fulfill (11), we may continue until after $j$ steps

$$
G_{1}^{(j)}>-\frac{d}{4^{j}}>-1 \text { for some } j>1
$$

where inductively $G_{1}^{(n+1)}=G_{1}^{(n)}+\frac{1}{d_{n}}\left(G_{1}^{(n)}\right)^{2}, d_{n}:=-\inf G_{1}^{(n)}$

We then set

$$
\mathrm{G}_{2}=\mathrm{G}_{1}^{(\mathrm{j})}
$$

(11) stays also true for

$$
G_{3}(p):=G_{2}^{2}(p) \geq 0
$$

Since $G_{3}$ is a nontrivial analytic function, it has only finitely many zeroes in any compact region of space. We may thus choose $\mu \geq 1$ such that

$$
\mathrm{g}_{0}(\mathrm{q}):=\frac{1}{\mathrm{G}_{3}(0)} \mathrm{G}_{3}\left(\frac{\mathrm{q}}{\mu}\right)>\frac{1}{2} \text { for } \mathrm{q}:=|\mathrm{p}| \leq 2
$$

Choosing $B \geq 2$ we thus have for $1 \leq q \leq q_{1}$ (where $q_{i}=\left|p_{i}\right|$ )

$$
\frac{1}{B q^{4}}<g_{0}(p), \frac{1}{B q_{1}^{4}}=g_{0}\left(p_{1}\right) \text { for some } q_{1}>2
$$

Let

$$
g_{1}(p)=\frac{1}{q_{1}^{4}} g_{0}\left(\frac{p}{q_{1}}\right)+g_{0}(p)
$$

Then

$$
\frac{1}{B q^{4}}<g_{1}(p), \frac{1}{B q_{2}^{4}}=g_{1}\left(p_{2}\right) \text { for } 1 \leq q \leq q_{2} \text { with } q_{2}>q_{1}^{2}
$$

Defining inductively $\mathrm{q}_{\mathrm{n}}$ such that $\mathrm{g}_{\mathrm{n}-1}\left(\mathrm{q}_{\mathrm{n}}\right)=\frac{1}{B \mathrm{q}_{\mathrm{n}}^{4}}$ and

$$
g_{n}(p)=\frac{1}{q_{n}^{4}} g_{0}\left(\frac{p}{q_{n}}\right)+g_{n-1}(p) \quad q_{n}>q_{1}^{n}>2^{n}
$$


we find that

$$
g_{\infty}(p)=\lim _{n \rightarrow \infty} g_{n}(p)>\frac{1}{B q_{n}^{4}} \quad \text { for } q>1
$$

By standard uniform convergence arguments it is also smooth and has vanishing derivatives up to order $2 \mathrm{~L}$ at 0 and it fulfills the analogue of (11). Its Fourier transform still vanishes for $|x|>1 / \mu 2^{j+1} M$. We also have an upper bound on $g_{\infty}$ :

Choose $b<\left(\frac{1}{2}\right)^{7}$ such that

$$
\mathrm{g}_{0}<\frac{1}{\mathrm{bq}^{8}} \quad \forall \mathrm{q}>1
$$

We then find for $\mathrm{q}_{\mathrm{n}} \leq \mathrm{q}<\mathrm{q}_{\mathrm{n}+1}$ (with $\mathrm{q}_{0}=1$ )

$$
\begin{aligned}
g_{\infty}(p) & <\frac{1}{b}\left(\frac{1}{q^{8}}+\frac{1}{q_{1}^{4}}\left(\frac{q_{1}}{q}\right)^{8}+\ldots+\frac{1}{q_{n}^{4}}\left(\frac{q_{n}}{q}\right)^{8}+\frac{1}{q_{n+1}^{4}}+\frac{1}{q_{n+2}^{4}} \ldots\right) \\
& <\frac{1}{b q^{4}}\left(\frac{1}{q^{4}}+\left(\frac{q_{1}}{q}\right)^{4}+\ldots+\left(\frac{q_{n}}{q}\right)^{4}+\left(\frac{q}{q_{n+1}}\right)^{4}+\ldots\right) \\
& <\frac{1}{b q^{4}} 2 \sum_{m=0}^{\infty}\left(\frac{1}{2^{4}}\right)^{m}<\frac{3}{b q^{4}} .
\end{aligned}
$$

We finally set

$$
g(p)=\frac{g_{\infty}(p)}{g_{\infty}(0)} \quad f_{1}=\frac{1}{g}-1
$$

Then all estimates on $f_{1}$ are immediate consequences of the established properties of $g$. On making once more a scale transformation on $\mathrm{g}, \mathrm{f}_{1}$ with a scale parameter $\mu>1$ one finds that the constants may be scaled as $(\alpha, \mathrm{A}) \rightarrow \mu^{-4}(\alpha, \mathrm{A}), \mathrm{C} \rightarrow 1 / \mu \mathrm{C}$ and thus may be chosen arbitrarily small.

(ii) is trivial.

QED

As was already mentioned we also have to introduce an infrared (IR) regularization to make the expressions we handle meaningful. This regularization will be removed later on with the help of the cluster and Mayer expansions. There is, of course, a lot of arbitrariness in introducing such a regularization, in our case even more than e.g. for a $\mathrm{P}(\varphi)$-theory, since our det-interaction is highly nonlocal. Roughly speaking the system will be enclosed in a (large) box : We choose a square 


\section{$\Lambda \subset \mathbb{R}^{2}$}

centered at the origin with volume

$$
|\Lambda|=4 n^{2}>1, \quad n \in \mathbb{N}
$$

More details will be given below (see (25),(26)).

We now use the $U V$ regulator $f_{\rho}$ to define a regularized $\sigma$-covariance. We will fix the choice of the parameter $\rho$ in (7) later $(\operatorname{see}(61))$ and call the functions $f_{\rho}, \hat{f}_{\rho}$ with that choice simply $f, \hat{f}$. We shall then denote by

$$
\mathrm{d} \mu_{\mathrm{f}}(\sigma)
$$

the Gaussian measure with mean zero and covariance

$$
\left(\frac{1}{1+\hat{f}}\right)(\mathrm{x}, \mathrm{y}) \text { or }\left(\frac{1}{1+\hat{\mathrm{f}}}\right)(\mathrm{x}-\mathrm{y}) \text {. }
$$

$\mathrm{d} \mu_{\mathrm{f}}(\sigma)$ will replace in the rigorous definition of $S_{2}$ the term $\int \mathrm{D} \sigma \mathrm{e}^{-1 / 2 \int \sigma^{2}}$ from (4). Since we will always keep the UV-cutoffs the $\sigma$ field may be viewed as an element of $\mathfrak{L}^{2}(\Lambda)$ [6] (or even as a differentiable function if we restrict $g(p)$ in (9) to fall off more rapidly for large $p$. If we want to take away the cutoff, $\sigma$ should be viewed as a distribution).

We now look at the determinant

$$
\operatorname{det}(\not p+g \sigma)
$$

in (4). Formally

$$
\operatorname{det}(\not p+g \sigma)=(\operatorname{det} p) \operatorname{det}\left(1+\frac{1}{p} g \sigma\right)
$$

We define $\chi_{\Lambda}$ to be the characteristic function of $\Lambda$ and set $\sigma=\sigma \chi_{\Lambda}=\sigma_{\Lambda^{\prime}}$. Replacing also $p \rightarrow p_{u}$ (6) $\frac{\sigma}{p_{u}}$ is trace class, and the second determinant makes sense. The first will be omitted since it is a global normalization factor which drops out on dividing by the partition function or vacuum functional (It may be interpreted as $\operatorname{det}^{-1}\left(\left(\frac{1}{p}\right)_{\Lambda}\right)$ ). It turns out, however, that for our purposes it is advantageous to rewrite 


$$
\operatorname{det}\left(1+\frac{1}{p} g \sigma\right)
$$

- where $\not p$ from now on always is $p_{u}$ - in a different form, namely as a determinant of a Hermitian operator, which is more suitable for the subsequent estimates. We will then restrict this operator to the volume $\Lambda$, which frees us from certain annoying boundary terms (the reader will realize that there are still enough terms of that sort left). We think this justified since one volume cutoff is in principle as good as another, and simplicity is a reasonable criterion.

We proceed as follows. Introducing

$$
\tau(\mathrm{x})=\sigma(\mathrm{x})-\sigma_{0}(\mathrm{x}) \text { with } 0 \leq \sigma_{0}(\mathrm{x}) \leq \sigma_{0} \in \mathbb{R}
$$

we may rewrite (13) as

$$
\operatorname{det}\left(1+\frac{1}{\not p} g \sigma\right)=\operatorname{det}\left(1+\frac{1}{\not p}\left(g \tau+g \sigma_{0}\right)\right)
$$

To make everything in (15) well-defined we suppose

$$
\tau, \sigma_{0} \in \mathcal{L}^{1}\left(\mathbb{R}^{2}\right)
$$

We also suppose $\sigma_{0}(\mathrm{x})$ to be constant in $\Lambda$ with value $\sigma_{0}$ and smoothly decreasing with $|\mathrm{x}|$. We call

$$
\mathrm{m}(\mathrm{x})=\mathrm{g} \sigma_{0}(\mathrm{x})
$$

Under the preceding assumptions we now prove the following

\section{Proposition 1}

$$
\operatorname{det}\left(1+\frac{1}{\not p}(g \tau+m)\right)=\operatorname{det}^{1 / 2}(1+\widetilde{A}+\widetilde{B}) \operatorname{det}\left(1+\frac{m}{\not p}\right)
$$

where

$$
\begin{aligned}
& \widetilde{A}=g \sigma \frac{1}{(-\not p+m)(p+m)} g \sigma-m \frac{1}{(-p+m)(\not p+m)} m \\
& \widetilde{B}=g \sigma \frac{1}{(-\not p+m)(p+m)} p-\not p \frac{1}{(-p+m)(p+m)} g \sigma
\end{aligned}
$$


Here and in the proof $\mathrm{m}$ stands for $\mathrm{m}(\mathrm{x})$, the associated operator on $\mathfrak{L}^{2}$.

Proof: Our assumptions guarantee $\frac{1}{p}(\mathrm{~g} \tau+\mathrm{m})$ to be trace class. We first assume $\mathrm{g}$ to be so small (for given $\tau$ and $\sigma_{0}$ ) that

$$
\operatorname{Tr} \ln \left(1+\frac{1}{p}(g \tau+\mathrm{m})\right)
$$

has a convergent expansion in $\mathrm{g}$. Then

$$
\operatorname{det}\left(1+\frac{1}{\not p}(g \tau+m)\right)=\exp \operatorname{Tr} \sum_{n=1}^{\infty}(-1)^{\mathrm{n}+1} \frac{1}{n}\left(\frac{1}{p}(g \tau+m)\right)^{\mathrm{n}}
$$

Since det is an entire function of $\mathrm{g}$ [7] one may then convince oneself that (17) also holds for arbitrary $\mathrm{g}$ by analytic continuation, once it is established for sufficiently small $\mathrm{g}$.

We find for $\mathrm{g}$ sufficiently small (remember $\mathrm{m}=\mathrm{g} \sigma_{0}$ )

$$
\begin{aligned}
\operatorname{det}\left(1+\frac{1}{\not p}(g \tau+\mathrm{m})\right) & =\exp \operatorname{Tr} \sum_{\mathrm{n}} \frac{(-)^{\mathrm{n}+1}}{\mathrm{n}}\left(\frac{1}{\not p}(\mathrm{~g} \tau+\mathrm{m})\right)^{\mathrm{n}} \\
& =\exp \operatorname{Tr} \sum_{\mathrm{n}} \frac{(-1)}{2 \mathrm{n}}\left(\frac{1}{\not p}(\mathrm{~g} \tau+\mathrm{m})\right)^{2 \mathrm{n}} \\
& =\exp \operatorname{Tr} \sum_{\mathrm{n}} \frac{(-1)}{2 \mathrm{n}}\left((\mathrm{g} \tau+\mathrm{m}) \frac{1}{-\not p}\right)^{2 \mathrm{n}} \\
& =\operatorname{det}\left(1+(\mathrm{g} \tau+\mathrm{m}) \frac{1}{-\not p}\right)
\end{aligned}
$$

Here we used that the fact that the trace of an odd number of $\frac{1}{p}$ 's vanishes. The last det is that of the adjoint of the first. We therefore know for $g$ small and real

$$
\operatorname{det}\left(1+\frac{1}{\not p}(g \tau+m)\right)>0
$$

This together with $(20)$ and

$$
\operatorname{det}\left(1+\frac{1}{p}(g \tau+m)\right)=\operatorname{det}\left(\frac{p+m}{p}\right) \operatorname{det}\left(1+\frac{1}{p+m} g \tau\right)
$$


(and similarly for the adjoint) now tells us

$$
\begin{aligned}
& \operatorname{det}\left(1+\frac{1}{\not p}(g \tau+m)\right)=\operatorname{det}^{1 / 2}\left(\frac{p+m}{p}\right) \operatorname{det}^{1 / 2}\left(\frac{-\not p+m}{-p}\right) \times \\
& \operatorname{det}^{1 / 2}\left(1+\frac{1}{p+m} g \tau+g \tau \frac{1}{-p+m}+g \tau \frac{1}{-p+m} \frac{1}{p+m} g \tau\right)
\end{aligned}
$$

The first two $\operatorname{det}^{1 / 2}$ 's give the second term on the r.h.s. of (17). The last determinant may be reexpressed in terms of $\sigma$ as

$$
\begin{aligned}
& \operatorname{det}^{1 / 2}\left(1+\frac{1}{\not p+m}(g \sigma-m)+(g \sigma-m) \frac{1}{-\not p+m}+(g \sigma-m) \frac{1}{-\not p+m} \frac{1}{\not p+m}(g \sigma-m)\right) \\
& =\operatorname{det}^{1 / 2}\left(1+(-\not p+m) \frac{1}{(-\not p+m)(p+m)}(g \sigma-m)+(g \sigma-m) \frac{1}{(-\not p+m)(p+m)}(\not p+m)+\right. \\
& \left.+(g \sigma-m) \frac{1}{(-p+m)(p+m)}(g \sigma-m)\right) \\
& =\operatorname{det}^{1 / 2}\left(1+(-\not p) \frac{1}{(-p+m)(p+m)} g \sigma+g \sigma \frac{1}{(-p+m)(p+m)} p+\right. \\
& \left.+g \sigma \frac{1}{(-\not p+m)(\not p+m)} g \sigma-m \frac{1}{(-\not p+m)(\not p+m)} m\right)
\end{aligned}
$$

This establishes (17) for g small and real.

To establish the relation for any real and positive $g$ note that $\operatorname{det}(1+\widetilde{\mathrm{A}}+\widetilde{\mathrm{B}})$ is analytic in $\mathrm{g}$ and that for $g$ real

$$
1+\widetilde{A}+\widetilde{B}=\left(1+F^{*}\right)(1+F) \geq 0 \quad \text { with } \quad F=\frac{1}{p p+m} g \tau
$$

Therefore $\operatorname{det}(1+\widetilde{\mathrm{A}}+\widetilde{\mathrm{B}}) \geq 0$, and any zero on the positive real $\mathrm{g}$-axis has to be of even order. Then $\operatorname{det}^{1 / 2}(1+\widetilde{\mathrm{A}}+\widetilde{\mathrm{B}})$ is also analytic in a neighbourhood of the positive real axis and (17) holds for any $g>0$ by analytic continuation, thus in particular for $g=\sqrt{\lambda / N}$ (which, in fact, is very small !).

QED

With the aid of the proposition we now come to the definition of the IR-regularized determinant. We set 


$$
\begin{aligned}
& \mathrm{A}=\chi_{\Lambda}\left(\mathrm{g} \sigma \frac{1}{\mathrm{p}^{2}+\mathrm{m}^{2}} \mathrm{~g} \sigma-\frac{\mathrm{m}^{2}}{\mathrm{p}^{2}+\mathrm{m}^{2}}\right) \chi_{\Lambda} \\
& \mathrm{B}=\chi_{\Lambda}\left(\mathrm{g} \sigma \frac{1}{\mathrm{p}^{2}+\mathrm{m}^{2}} p-p \frac{\mathrm{m}^{2}}{\mathrm{p}^{2}+\mathrm{m}^{2}} \mathrm{~g} \sigma\right) \chi_{\Lambda}
\end{aligned}
$$

Here and from now on $\mathrm{m}$ denotes $\mathrm{m}(0)=\mathrm{g} \sigma_{0}$, i.e. we have taken the limit of constant mass, which is possible after introducing the $\chi_{\Lambda}$. The value of $m$ will be fixed later.

The support of the field variable $\sigma$ in the interaction determinant is restricted to $\Lambda$. At a later stage we will take $\Lambda \rightarrow \infty$. Denoting $\widehat{\Lambda}=\mathbb{R}^{2} \backslash \Lambda$ we include (for technical simplification later on) also a term

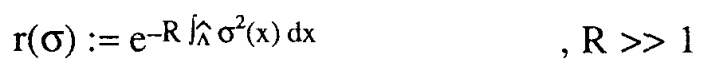

and take $\mathrm{R} \rightarrow \infty$ once we have performed the expansion. (26) makes explicit that interactions with $\widehat{\Lambda}$ are suppressed. (Absorbing (26) in the covariance would replace, for $R \rightarrow \infty$,

$$
\left.\left(\frac{1}{1+\hat{\mathrm{f}}}\right)(\mathrm{x}, \mathrm{y}) \rightarrow \chi_{\Lambda}(\mathrm{x})\left(\frac{1}{1+\hat{\mathrm{f}}}\right)(\mathrm{x}, \mathrm{y}) \chi_{\Lambda}(\mathrm{y})\right)
$$

Having fixed the way in which we introduce the finite volume we also have to say a word on the boundary conditions (b.c.) on $\partial \Lambda$ : According to the Peierls argument [8] the b.c. are decisive for the realization of one of the two phases in the two phase region. To fix them - and for later use we introduce a lattice of (closed) unit squares with corner coodinates in $\mathbb{Z}^{2}$, called

$$
\Delta\left(\mathrm{z}_{1}, \mathrm{z}_{2}\right) \text { or } \Delta_{\mathrm{j}} \text { or } \Delta
$$

with $\Delta \subset \Lambda, \cup=\Lambda$ and demand :

$\Delta$

If $\Delta \cap \partial \Lambda \neq \emptyset: \sigma(\Delta):=\int_{\Delta} \sigma \geq 0$

More precisely, all $\sigma$-functional integrals in the following will contain a term $b(\sigma)$ (b for boundary)

$$
\mathrm{b}(\sigma):=\sum_{\Delta \cap \partial \Lambda \neq \varnothing} \theta(\sigma(\Delta)-1 / 4)
$$


where $\theta(\mathrm{x})$ is (for technical reasons) a smoothed step function

$$
\theta(x)=\left\{\begin{array}{ll}
0 & x \leq-1 / 4 \\
1 & x \geq 1 / 4
\end{array},\right. \text { monotonic and smooth }
$$

Of course we could equally well study the case with opposite sign of $\sigma(\Delta)$. And we can also fix the value of $\sigma$ near the boundary by different rules to obtain the same result, but we do not intend to discuss this problem here in full generality. Obviously the b.c. (27) favours positive expectation values of $\sigma$, and we shall indeed find out

$$
<\sigma>>0 \quad \text { (see Proposition 4) }
$$

Using the regulators and the b.c. we may now make an attempt to define the two point function $S_{2}(x, y)$ in a rigorous way. For technical reasons it will be sometimes helpful (but not very important) to smear it out with test functions $\mathrm{f}_{1}, \mathrm{f}_{2}$. We assume them to be real $\mathcal{L}^{2}$-functions and (without restriction) to have their support only in a single square $\Delta\left(f_{1}\right)\left(\operatorname{resp} . \Delta\left(f_{2}\right)\right)$ which are of course arbitrary and may be varied. Since we are working in a $2 \times \mathrm{N}$-component space they strictly speaking will be assumed to be of the form

$$
\left(0, \ldots, 0, f_{j}^{(1)}, f_{j}^{(2)}, 0, \ldots, 0\right), \quad j=1,2
$$

where the entries are in position $2 \mathrm{i}-1,2 \mathrm{i}$ of the $2 \mathrm{~N}$-vector. Finally we assume (again without restriction) that

$$
<f_{j}, f_{j}>:=\int f_{j}^{*}(x) f_{j}(x) d^{2} x=1
$$

We thus now define the unnormalized UV-regularized two-point function in the volume $\Lambda$ and the respective partition function as

$$
S_{2 \text { un }}^{\Lambda}\left(f_{1}, f_{2}\right)=\int d \mu_{f}(\sigma)<f_{1}, \frac{1}{\not p+g \sigma} f_{2}>\operatorname{det}^{1 / 2}(1+A+B) b(\sigma) r(\sigma)
$$

$$
Z^{\Lambda} \quad=\int d \mu_{f}(\sigma) \operatorname{det}^{1 / 2}(1+A+B) b(\sigma) r(\sigma)
$$


(we used (12), (17), (25)-(27), formally starting from (4), and we formally divided S,Z by $\operatorname{det}(\not p+m)) . \not p$ denotes $p_{u}(6), p^{2}=\left(-p_{u}\right) p_{u}$.

To estimate the finite volume quantities and to be able to take the infinite volume limit later on we have to perform the usual cluster and Mayer expansions. Since the regions where $\sigma$ is far from either of the minima of the potential, are highly suppressed in probability, we shall estimate them directly without expanding the couplings between squares within a given connected component of such a region. This means that our expansion will be defined differently for different sets of $\sigma$ - configurations. The Hilbert-Schmidt norm of the operator A (25) restricted to a given square $\Delta$ turns out to be an appropriate criterion to distinguish between the small field (near the minima) and large field (highly suppressed) regions. Thus for every square $\Delta$ in $\Lambda$ we shall introduce in the functional integral a factor

$$
1_{\Delta}=\theta\left(\frac{\left\|A_{\Delta}\right\|_{2}}{N^{\alpha}}-1\right)+\left(1-\theta\left(\frac{\left\|A_{\Delta}\right\|_{2}}{N^{\alpha}}-1\right)\right)
$$

$\left(\right.$ see $\left.\left(27^{\prime}\right)\right)$

and then expand the product over the $\Delta$ 's (see (47)). We use the following

\section{Definition :}

Given any $\sigma$-configuration with $\sigma \in \mathcal{L}^{2}(\Lambda)$ we call $\Delta \subset \Lambda$ a large field or $\ell$-square if it carries a factor

$$
\theta_{\Delta}^{\ell}(\sigma)=\theta\left(\frac{\|\mathrm{A} \Delta\|_{2}}{N^{\alpha}}-1\right) \quad \text { where }
$$

$$
\left\|\mathrm{A}_{\Delta}\right\|_{2}=\left\|\chi_{\Delta} \mathrm{A} \chi_{\Delta}\right\|_{2} \quad \text { (Hilbert-Schmidt-Norm), (in contrast to the operator norm }
$$

$\left.\left\|A_{\Delta}\right\|\right)$, and we call it a small field or s-square, if it carries a factor

$$
\theta_{\Delta}^{\mathrm{s}}(\sigma)=1-\theta\left(\frac{\|\left.\mathrm{A}_{\Delta}\right|_{2}}{\mathrm{~N}^{\alpha}}-1\right)=1-\theta_{\Delta}^{\ell}(\sigma)
$$

Note that if the Hilbert-Schmidt-norms $\left\|\mathrm{A}_{\Delta}\right\|_{2}$ in (30), (31) are bounded by $\mathrm{O}(1) \mathrm{N}^{\alpha}$, then the corresponding operator norms are bounded by $\mathrm{O}(1) \mathrm{N}^{(\alpha-1 / 2)}$ due to the $\mathrm{N}$-fold degeneracy, i.e. they are much smaller than 1 with the choice (34). 
For an s-square $\Delta$ we write ${ }^{2}$

$$
\left(1-\theta_{\Delta}^{\ell}(\sigma)\right)=\left(1-\theta_{\Delta}^{\ell}(\sigma)\right) \theta(\sigma(\Delta))+\left(1-\theta_{\Delta}^{\ell}(\sigma)\right)(\theta(-\sigma(\Delta)))
$$

where $\sigma(\Delta)=\int_{\Delta} \sigma(\mathrm{x}) \mathrm{d}^{2} \mathrm{x}$.

And we call an s-square

$$
\mathrm{s}_{ \pm}, \text {if it carries a factor }\left(1-\theta_{\Delta}^{\ell}(\sigma)\right) \theta( \pm \sigma(\Delta))=: \theta_{\Delta}^{\mathrm{S}_{ \pm}}(\sigma)
$$

$\alpha$ in (30), (31) has to be chosen between 0 and $1 / 6$, for definiteness

$$
\alpha=\frac{1}{10}
$$

For technical reasons it is (unfortunately) necessary to define several kinds of large field regions by successively enlarging the region of $\ell$-squares :

Definition : Let $\ell$ and s be the set of large and small field squares for a given $\sigma(\mathrm{x})$ (see (30)(33))

$$
\begin{array}{ll}
\ell \cup \mathrm{s}=\Lambda & \ell \cap \mathrm{s}=0 \\
\mathrm{~s}=\mathrm{s}_{+} \cup \mathrm{s}_{-} & \mathrm{s}_{+} \cap \mathrm{s}_{-}=0
\end{array}
$$

0 in set-theoretic relations always denotes a set of $\mathfrak{L}^{2}$-measure 0 . The next step is to define $\ell_{1} \supset \ell$ through $\Delta \in \ell_{1}$, if $\Delta \in \ell$ or if $\Delta \in \mathrm{s}_{ \pm}$and there exists $\Delta^{\prime} \in \mathrm{s}_{\mp}, \Delta^{\prime} \neq \Delta$ such that $\Delta$ and $\Delta^{\prime}$ have a common edge.

Accordingly

$$
\mathrm{s}_{1}=\Lambda \backslash \ell_{1}, \mathrm{~s}_{1}=\mathrm{s}_{1+} \cup \mathrm{s}_{1-}, \mathrm{s}_{1 \pm} \subset \mathrm{s}_{ \pm}
$$

Then we define $\ell_{2} \supset \ell_{1}$ through :

$$
\Delta \in \ell_{2}, \text { if } \Delta \in \ell_{1} \text { or if } \operatorname{dist}\left(\Delta, \ell_{1}\right)=0
$$

\footnotetext{
2 (32) also holds if we do not assume $\theta(x)+\theta(-x)=1$, since $1-\theta_{\Delta}^{\ell}(\sigma)$ vanishes for $|\sigma(\Delta)| \leq 1 / 4$ (Lemma 14).
} 
and accordingly

$$
\mathrm{s}_{2}=\Lambda \backslash \ell_{2}, \mathrm{~s}_{2}=\mathrm{s}_{2+} \cup \mathrm{s}_{2-}, \mathrm{s}_{2 \pm} \subset \mathrm{s}_{1 \pm}
$$

Now we define $\mathrm{L} \supset \ell_{2}$

$$
\Delta \in \mathrm{L} \text {, if } \Delta \in \ell_{2} \text { or if } \operatorname{dist}\left(\Delta, \ell_{2}\right)=0
$$

and correspondingly $S, S_{+}$and $S_{-}$.

For given $\ell_{1}$ we define

$$
\Gamma=\left\{\Delta \subset \Lambda \mid \operatorname{dist}\left(\Delta, \ell_{1}\right) \leq \mathrm{M}\right\}
$$

where we choose (for definiteness)

(40) $\quad \mathrm{M}=\frac{2}{\mathrm{~m}} \log \mathrm{N}>1$.

The set $\ell_{1}$ is also split into connectivity components as follows :

For $\Delta_{i}, \Delta_{j} \in \ell_{1}$ we say there is a connectivity link between $\Delta_{i}, \Delta_{j}$ if there exists $\Delta \subset \Lambda$ such that

$$
\operatorname{dist}\left(\Delta_{\mathrm{i}}, \Delta\right)+\operatorname{dist}\left(\Delta_{\mathrm{j}}, \Delta\right) \leq 2 \mathrm{M}
$$

By $\ell_{11}, \ldots, \ell_{1 \mathrm{n}}$ we denote the maximal subsets of $\ell_{1}$, connected by connectivity links, and call these connectivity components. This splitting induces a corresponding one of $\ell_{2}$ and $\mathrm{L}$ into $\ell_{21}, \ldots, \ell_{2 \mathrm{n}}$ and $\mathrm{L}_{1}, \ldots, \mathrm{L}_{\mathrm{n}}$.

There is a one-to-one relation between $\ell_{11}, \ldots, \ell_{1 \mathrm{n}}$ and the corresponding subsets of $\Gamma$, called connectivity components of $\Gamma$ :

$$
\ell_{1 \mathrm{a}} \subset \Gamma_{\mathrm{a}} \subset \Gamma, \text { with }
$$

$$
\Gamma_{\mathrm{a}}=\left\{\Delta \mid \operatorname{dist}\left(\ell_{1 \mathrm{a}}, \Delta\right) \leq \mathrm{M}\right\},
$$
so that 


$$
\Gamma_{\mathrm{a}} \cap \Gamma_{\mathrm{b}}=0, \mathrm{a} \neq \mathrm{b}, \bigcup_{\mathrm{a}=1}^{\mathrm{n}} \Gamma_{\mathrm{a}}=\Gamma
$$

Finally we introduce

$$
\begin{aligned}
& \gamma_{\mathrm{a}}=\left\{\Delta \subset \Lambda \mid \operatorname{dist}\left(\Delta, \ell_{1 \mathrm{a}}\right) \leq \frac{\mathrm{M}}{2}\right\} \cup\left\{\mathrm{x} \in \hat{\Lambda} \mid \operatorname{dist}\left(\mathrm{x}, \ell_{1 \mathrm{a}}\right) \leq \frac{\mathrm{M}}{2}\right\} \\
& \gamma=\underset{\mathrm{a}}{\cup} \gamma_{\mathrm{a}}
\end{aligned}
$$

so that

(44) $\quad \operatorname{dist}\left(\gamma, \Gamma^{\prime}\right) \geq \frac{\mathrm{M}}{2}-\sqrt{2}$,

where we always denote for any set $E \subset \mathbb{R}^{2}$

$$
E^{\prime}=\Lambda \mathrm{E}, \widehat{E}=\mathbb{R}^{2} \backslash E
$$

The full hierarchy of large field regions is thus

$$
\ell \subset \ell_{1} \subset \ell_{2} \subset \mathrm{L} \subset(\gamma \cap \Lambda) \subset \Gamma, \ell_{1 \mathrm{a}} \subset \mathrm{L}_{\mathrm{a}} \subset \gamma_{\mathrm{a}} \cap \Lambda \subset \Gamma_{\mathrm{a}}
$$

$\left(\mathrm{L}_{\mathrm{a}}\right.$ are introduced with respect to $\ell_{1 \mathrm{a}}$ as in (38)).

All squares $\Delta$ fulfill $\Delta \subset \Lambda . \widehat{\Lambda}$ will be viewed as a single (large field) block.

Now we go back to (29). We reexpress

$$
\begin{gathered}
\mathrm{S}_{2 \text { un }}^{\Lambda}=\sum_{\ell, \mathrm{s}^{+} \subset \Lambda} \int_{\mathrm{f}} \mathrm{d} \mu_{\mathrm{f}}(\sigma)<\mathrm{f}_{1}, \frac{1}{\mathrm{p}+\mathrm{g} \sigma} \mathrm{f}_{2}>\operatorname{det}^{1 / 2}(1+\mathrm{K}) \mathrm{b}(\sigma) \mathrm{r}(\sigma) \\
\quad \times \prod_{\Delta \in \ell} \theta_{\Delta}^{\ell}(\sigma) \prod_{\Delta \in \mathrm{s}^{+}} \theta_{\Delta}^{\mathrm{s}^{+}}(\sigma) \prod_{\Delta \in \mathrm{s}^{-}} \theta_{\Delta}^{\mathrm{s}^{-}}(\sigma)
\end{gathered}
$$

(see (27'), (30) - (31)).

(The sum is over subsets of squares $\ell, \mathrm{s}^{+}$with $\ell \cap \mathrm{s}^{+}=0, \mathrm{~s}^{-}=\Lambda \backslash\left\{\ell \cup \sigma^{+}\right\}$(as set of $\left.\Delta^{\prime} \mathrm{s}\right)$ ). 


$$
\mathrm{K}=\mathrm{A}+\mathrm{B} \quad(\operatorname{see}(25))
$$

As mentioned above $\mathrm{K}$ can be viewed as an Hilbert-Schmidt-operator on $\mathfrak{d}^{2}(\Lambda)$. In the following we will use the same symbol for an operator and its position space kernel, indicating arguments explicitly if necessary $(\mathrm{K}(\mathrm{x}, \mathrm{y}), \ldots)$.

The subsequent treatment of (47) will depend crucially on whether we are in the regions L or $\mathrm{S}$. The representation in terms of $\mathrm{K}(48)$ is suited for $\mathrm{L}$, but not for $\mathrm{S}$. We therefore rewrite $\operatorname{det}^{1 / 2}(1+K)$ in an (unfortunately) more lengthy form.

We introduce the operators

$$
\mathrm{K}_{+}=\mathrm{P}_{+} \mathrm{KP} \mathrm{P}_{+}, \mathrm{K}_{-}=\mathrm{P}_{-} \mathrm{KP} \mathrm{P}_{-}, \mathrm{A}_{\mathrm{L}_{\mathrm{a}}}=\mathrm{P}_{\mathrm{L}_{\mathrm{a}}} A \mathrm{P}_{\mathrm{L}_{\mathrm{a}}}
$$

where the P's denote the projections on $S_{+}, S_{-}, L_{a}$, i.e. in position space multiplication by $\chi_{S_{+}}$, $\chi_{S_{-}}, \chi_{L_{a}}$. Going back to $F(\tau)(24)$ and defining

$$
F_{+}(\tau):=F P_{+}=\frac{1}{p+m} g \tau_{+}, F_{-}(\tau):=F P_{-}:=\frac{1}{\not p-m} g \tau
$$

$\left(\tau_{ \pm}:=\left(\sigma \mp \sigma_{0}\right) P_{ \pm}\right)$

we have (see (24)), using the cyclicity of the trace,

$$
\operatorname{Tr} \mathrm{K}_{ \pm}=\operatorname{Tr}\left(\mathrm{F}_{ \pm}+\mathrm{F}_{ \pm}^{*}+\mathrm{F}_{ \pm} \mathrm{F}_{ \pm}^{*}\right)
$$

$\operatorname{Tr} K_{+}^{2}=\operatorname{Tr}\left(F_{+}^{2}+F_{+}^{*^{2}}+F_{+} F_{+}^{*}+F_{+}^{*} F_{+}+2 F_{+}^{2} F_{+}^{*}+2 F_{+} F_{+}^{*^{2}}+\left(F_{+} F_{+}^{*}\right)^{2}\right)$ (analogously for $+\rightarrow-$ ) and

$$
\begin{aligned}
& \operatorname{Tr}\left(\mathrm{F}_{+}^{2} \mathrm{~F}_{+}^{*}+\mathrm{F}_{+} \mathrm{F}_{+}^{* 2}\right)=2 \mathrm{~g}^{3} \operatorname{Tr}\left(\tau_{+} \frac{1}{\mathrm{p}^{2}+\mathrm{m}^{2}} \tau_{+} \frac{\mathrm{m}}{\mathrm{p}^{2}+\mathrm{m}^{2}} \tau_{+}\right) \\
& \operatorname{Tr}\left(\left(\mathrm{F}_{+} \mathrm{F}_{+}\right)^{* 2}\right)=\mathrm{g}^{4} \operatorname{Tr}\left(\tau_{+}^{2} \frac{1}{\mathrm{p}^{2}+\mathrm{m}^{2}} \tau_{+}^{2} \frac{1}{\mathrm{p}^{2}+\mathrm{m}^{2}}\right) .
\end{aligned}
$$

From this we obtain (using $\operatorname{Tr} F=\operatorname{Tr} F^{*}, \operatorname{Tr} F^{2}=\operatorname{Tr} F^{* 2}(20)$ ) 


$$
\begin{aligned}
\operatorname{det}^{1 / 2}(1+\mathrm{K})= & \mathrm{e}^{\operatorname{Tr}\left(\mathrm{F}_{+}+\mathrm{F}_{-}-1 / 2\left(\mathrm{~F}_{+}^{2}+\mathrm{F}_{-}^{2}\right)\right) \times} \\
& \mathrm{e}^{-1 / 2 \operatorname{Tr}\left(\mathrm{F}_{+}^{2} \mathrm{~F}_{+}^{*}+\mathrm{F}_{+} \mathrm{F}_{+}^{*}+1 / 2\left(\mathrm{~F}_{+} \mathrm{F}_{+}^{*}\right)^{2}+(+\leftrightarrow-)\right) \times} \\
& \mathrm{e}^{-1 / 2 \operatorname{Tr}\left(\mathrm{K}_{+}+\mathrm{K}_{-}-1 / 2\left(\mathrm{~K}_{+}^{2}+\mathrm{K}^{2}\right)\right) \times} \\
& \operatorname{det}^{1 / 2}(1+\mathrm{K})
\end{aligned}
$$

The last two terms may be rewritten as

$$
\begin{aligned}
& \operatorname{det}^{1 / 2}\left(1+\sum_{\mathrm{a}} \mathrm{A}_{\mathrm{L}_{\mathrm{a}}}+\mathrm{K}_{+}+\mathrm{K}_{-}+\mathrm{K}^{\prime}\right) \mathrm{e}^{-1 / 2 \operatorname{Tr}\left(\mathrm{K}_{+}+\mathrm{K}_{-}-1 / 2\left(\mathrm{~K}_{+}^{2}+\mathrm{K}_{-}^{2}\right)\right)} \\
& =\prod_{\mathrm{a}} \operatorname{det}^{1 / 2}\left(1+\mathrm{A}_{\mathrm{L}_{\mathrm{a}}}\right) \operatorname{det}_{3}^{1 / 2}\left(1+\mathrm{K}_{+}\right) \operatorname{det}_{3}^{1 / 2}\left(1+\mathrm{K}_{-}\right) \operatorname{det}^{1 / 2}(1+\mathrm{Q}),
\end{aligned}
$$

where we set

$$
K^{\prime}=K-\sum_{a} A_{L_{a}}-K_{+}-K_{-}, Q=\frac{1}{1+\sum_{a} A_{L_{a}}+K_{+}+K_{-}} K^{\prime}
$$

In (54) we used cyclicity and the pairwise orthogonality of the $A_{L_{a}}, K_{+}$and $K_{-}$. Q is well-defined since $A_{L_{a}}>-1$ (see Lemma 17 below), and since $\left\|K_{ \pm}\right\|<<1$ due to the small field condition. We also introduced the (standard) definition [7]

$$
\operatorname{det}_{r+1}(1+K):=\exp \left(\sum_{n=1}^{r}(-1)^{n} \frac{1}{n} \operatorname{Tr} K^{n}\right) \operatorname{det}(1+K)
$$

So far we have only introduced the auxiliary covariance (12) made up of the ultralocal $\sigma^{2}$ term and the regulator. The true covariance should contain (up to corrections, which are small in the expansion parameter $\mathrm{N}$ ) all terms quadratic in $\sigma$. Otherwise the expansion with respect to the covariance will not converge (or cannot be shown to do so). This does not apply to the large field region, however, since this region can be shown directly to be highly suppressed in probability.

As a first step towards the new covariance (which obviously then will depend on the configurations $\ell$,s) we define a new variable $\zeta$ instead of $\sigma$ which is shifted by $\mp \sigma_{0}$ in the regions $S_{ \pm}$. A suitable choice for $\sigma_{0}$ then guarantees that we expand around the minima of the interaction in both regions $\mathrm{S}_{+}$and $\mathrm{S}_{-}$, i.e. small $\zeta$ implies small deviations from the minima.

Due to the cutoff for the $\sigma$-field we have, however, to smooth this shifting of variable, since 
otherwise we get hardly controllable contributions from the cutoff. We therefore introduce smoothed characteristic functions of the squares $\Delta$ through the following steps :

$\alpha)$ Choose in one dimension a smooth function $\varphi\left(x_{1}\right)$ with

$$
\begin{aligned}
& 0 \leq \varphi\left(\mathrm{x}_{1}\right) \leq 1 \\
& \varphi\left(\mathrm{x}_{1}\right)= \begin{cases}1, & 1 / 4 \leq \mathrm{x}_{1} \leq 3 / 4 \\
0, & \mathrm{x}_{1} \leq-1 / 4, \mathrm{x}_{1} \geq 5 / 4\end{cases} \\
& \varphi\left(\mathrm{x}_{1}\right)+\varphi\left(\mathrm{x}_{1}+1\right)=1 \quad, \quad-1 / 4 \leq \mathrm{x}_{1} \leq 1 / 4 \\
& \varphi\left(1 / 2+x_{1}\right)=\varphi\left(1 / 2-x_{1}\right)
\end{aligned}
$$

and set

$$
\varphi_{\mathrm{n}_{1}}\left(\mathrm{x}_{1}\right)=\varphi\left(\mathrm{x}_{1}+\mathrm{n}_{1}\right), \quad \mathrm{n}_{1} \in \mathbb{Z}
$$

$\beta)$ Then we define for the square $\Delta$ with lower left corner $x_{\Delta}=\left(n_{1}, n_{2}\right)=n$ the smoothed characteristic function

$$
\varphi_{\left(\mathrm{n}_{1}, \mathrm{n}_{2}\right)}\left(\mathrm{x}_{1}, \mathrm{x}_{2}\right):=\varphi_{\mathrm{n}}(\mathrm{x}):=\varphi_{\Delta}(\mathrm{x}):=\varphi_{\mathrm{n}_{1}}\left(\mathrm{x}_{1}\right) \varphi_{\mathrm{n}_{2}}\left(\mathrm{x}_{2}\right)
$$

From (56) - (58) we find

$$
\sum_{n=\mathbb{Z}^{2}} \varphi_{n}(x) \equiv 1
$$

By construction we have for the Fourier transforms

$$
\tilde{\varphi}_{\Delta}(p)=\mathrm{e}^{\mathrm{ipx}} \tilde{\varphi}(\mathrm{p}) \text { and } \tilde{\varphi}(\mathrm{p}) \in \&\left(\mathbb{R}^{2}\right)
$$

We now go back to (7) - (9) and Lemma 1.

We choose $\mathrm{f}_{\rho}, \hat{\mathrm{f}}_{\rho}$ with $(7)-(9)$ such that $\widetilde{\mathrm{h}}_{\Delta}(\mathrm{p}):=\widetilde{\varphi}_{\Delta}(\mathrm{p}) \hat{\mathrm{f}}_{\rho}(\mathrm{p})$ fulfills

$$
\left|\widetilde{h}_{\Delta}(\mathrm{p})\right| \leq\left(\frac{1}{1+\mathrm{p}^{2}}\right)^{2} \mathrm{~N}^{-3 / 2}
$$

This is possible as will be shown now :

Assume $f_{1}$ to be given as in (9), (10). 
Choose $\mathrm{C}_{3}, \mathrm{C}_{4}(\mathrm{~L}) \geq 1$ such that

$$
F(p) \mid \leq\left\{\begin{array}{ll}
C_{3} & , q \leq C_{4} \\
q^{-2 L} & , q \geq C_{4}
\end{array} \quad(q=\mid p l)\right.
$$

Since

$$
f_{1}(p) \leq\left\{\begin{array}{lll}
A q^{2 L} & q<1 \\
A q^{4} & q>1
\end{array},\right.
$$

we have for $\rho^{1 / 2} \geq C_{4}$

$$
\left|\tilde{\varphi} f_{\rho}\right| \leq\left\{\begin{array}{ll}
C_{3} A\left(\frac{q}{\rho}\right)^{2 L} & , q \leq \rho^{1 / 2} \\
A q^{-2 L}\left(\frac{q}{\rho}\right)^{4} & , q \geq \rho^{1 / 2}
\end{array} .\right.
$$

From this we find (60) to be true if

$$
\rho=N^{3 /(2(L-2))}=N^{1 / 20},
$$

if $2 \mathrm{~A}$ is chosen smaller than 1 and $\mathrm{C}_{3}^{-1}$.

The important result is

Lemma 2 : The cutoff function for $\sigma, f:=f_{\rho}$ is fixed as follows

(i) Choose some $f_{1}$ according to (9)

(ii) Define $f(p)=f_{\rho}(p)=f_{1}(p / \rho)($ see $(61))$.

Then we have the estimate (60) and

$$
\int d^{2} p \frac{1}{1+f(p)}, \int d^{2} p \frac{1}{1+\hat{f}(p)} \leq O(1) N^{1 / 10}
$$

Here and in the following $\mathrm{O}(1)$ always denotes an $\mathrm{N}$-independent constant (which also does not 
exceed 1 by orders of magnitude...)

Remark: The $\mathrm{x}$-space support of $\frac{1}{1+\mathrm{f}}(9)$ will decrease with $\mathrm{N}$ proportional to $\mathrm{N}^{-1 / 20}$ so that we may safely assume

$$
\left(\frac{1}{1+f}\right)(x, y) \equiv 0 \quad \text { if }|x-y|>1
$$

Proof: (60) has just been established, (62) is a consequence of the lower bound on $\mathrm{f}_{1}$ in (9) and (61).

Now we define $\zeta(x)$ as

$$
\zeta(\mathrm{x})=\sigma(\mathrm{x})-\sigma_{0} \phi_{2+}+\sigma_{0} \phi_{2-}=: \sigma(\mathrm{x})-\sigma_{02+}(\mathrm{x})+\sigma_{02-}(\mathrm{x})
$$

where (see (37))

$$
\phi_{2 \pm}(\mathrm{x}):=\sum_{\Delta \in \mathrm{s}_{2+}} \varphi_{\Delta}(\mathrm{x}) \quad \text { (multiplication operators in position space) }
$$

So $\phi_{2 \pm}$ are not projectors as $\mathrm{P}_{+}, \mathrm{P}_{-}, \mathrm{P}_{\mathrm{L}}$ (49). But due to (56) - (59) we have

$$
\phi_{2 \pm}(x)= \begin{cases}1 & x \in S_{ \pm} \\ 0 & x \notin S_{ \pm}\end{cases}
$$

so that

$$
\frac{1}{p \pm m} g \tau_{ \pm}=\frac{1}{p \pm m} g \zeta_{ \pm}
$$

with $\zeta_{ \pm}=\zeta_{ \pm} \mathrm{P}_{ \pm}, \zeta_{\mathrm{L}}=\zeta \mathrm{P}_{\mathrm{L}}$ and similarly for $\sigma, \tau$

The relation between $\zeta_{\mathrm{L}}$ and $\sigma_{\mathrm{L}}$ is then

$$
\sigma_{\mathrm{L}}=\zeta_{\mathrm{L}}+\sigma_{0}\left(\phi_{2+}-\phi_{2-}\right) \mathrm{P}_{\mathrm{L}}
$$

Here the second term is sort of a boundary contribution which vanishes in $\ell_{1}$, but not in $L \backslash \ell_{1}$.

We may now reexpress $d \mu_{f}(\sigma)$ in terms of $d \mu_{f}(\zeta)$ : 


$$
\mathrm{d} \mu_{\mathrm{f}}(\sigma)=\mathrm{d} \mu_{\mathrm{f}}(\zeta) \exp \left\{-\frac{1}{2}\left\langle\left(\sigma_{02+}-\sigma_{02-}\right),(1+\hat{\mathrm{f}}) \zeta\right\rangle-\frac{1}{2}\left\langle\zeta,(1+\hat{\mathrm{f}})\left(\sigma_{02+}-\sigma_{02-}\right)\right\rangle\right.
$$

$$
\left.-\frac{1}{2}\left\langle\left(\sigma_{02+}-\sigma_{02-}\right),(1+\hat{f})\left(\sigma_{02+}-\sigma_{02-}\right)\right\rangle\right\}
$$

(where all terms in the second factor are restricted to $\Lambda$ ).

Due to $(64,65)$ we find

$$
\left\langle\left(\sigma_{02+}-\sigma_{02-}\right), \hat{\mathrm{f}} \zeta\right\rangle=\sigma_{0}\left\langle\sum_{\Delta \in \mathrm{s}_{2+}} \mathrm{h}_{\Delta}-\sum_{\Delta \in \mathrm{s}_{2-}} \mathrm{h}_{\Delta}, \zeta\right\rangle
$$

$$
=\sigma_{0}\left(\sum_{\Delta \in \mathrm{s}_{2+}}\left\langle\mathrm{h}_{\Delta}, \zeta\right\rangle-\sum_{\Delta \in \mathrm{s}_{2-}}\left\langle\mathrm{h}_{\Delta}, \zeta\right\rangle\right)
$$

As a result of $(60)$ we find :

$$
\mid<\mathrm{h}_{\Delta}, \zeta>1 \leq \mathrm{O}(1) \mathrm{N}^{-3 / 2}<\zeta, \zeta>^{1 / 2}
$$

The second term in (69) gives

$$
\begin{gathered}
<\sigma_{02+}-\sigma_{02-}, \hat{\mathrm{f}}\left(\sigma_{02+}-\sigma_{02-}\right)>=\sigma_{0}^{2}<\phi_{2+}-\phi_{2-}, \hat{\mathrm{f}}\left(\phi_{2+}-\phi_{2-}\right)> \\
=\sigma_{0}^{2}<\phi_{2+}-\phi_{2-}, \sum_{\Delta \in \mathrm{s}_{2+}} \mathrm{h}_{\Delta}-\sum_{\Delta \in \mathrm{s}_{2-}} \mathrm{h}_{\Delta}>
\end{gathered}
$$

and using (60) and the definition of $\phi_{2 \pm}$ we estimate

$$
\mathrm{k}_{2+}-\phi_{2-}, \sum_{\Delta \in \mathrm{s}_{2+}} \mathrm{h}_{\Delta-} \sum_{\Delta \in \mathrm{s}_{2-}} \mathrm{h}_{\Delta}>\mathrm{l} \leq \mathrm{O}(1)\left|\mathrm{s}_{2}\right| \mathrm{N}^{-3 / 2}
$$

Since $\sigma_{0} \sim N^{1 / 2}$ (see (76)) this implies that the perturbations caused by $f$ are small ; and this also ends the deviations necessitated by the $\sigma$-cutoff.

We noted before that, for the expansions to converge, it is necessary to include in the integration measure for $\zeta$ all those terms of the interaction which are quadratic in $\zeta$ and not suppressed by a negative power of $\mathrm{N}$ - as long as we stay in the small field region.

We therefore include the terms from (53) giving an interaction quadratic in $\zeta$ in the $S$ region. The 
parameter $\mathrm{m}$ will be fixed such that the terms linear in $\zeta$ from $\mathrm{F}_{ \pm}$cancel with the $\hat{\mathrm{f}}$-independent linear terms from the r.h.s. of (69). The higher order terms in $\zeta_{ \pm}$turn out to be small for large $N$ (in the small field region !) since they involve at least three powers of $g$ and thus contain a factor $\mathrm{N}^{-1 / 2}$ after taking into account the factor $\mathrm{N}$ from the flavour trace.

We thus proceed as follows. For the first exponential in (53) which contains the terms in question, we find

$\operatorname{Tr} F_{+}=\operatorname{Tr} \frac{1}{p+m} g \zeta_{+}=g N\left(\operatorname{Tr}_{2} \int \frac{1}{\not p+m} \frac{d^{2} p}{(2 \pi)^{2}}\right) \int_{s_{+}} \zeta_{+} d^{2} x=2 g N\left(\int \frac{m}{p^{2}+m^{2}} \frac{d^{2} p}{(2 \pi)^{2}}\right) \int \zeta_{+} d^{2} x$

$\left(\operatorname{Tr}_{2}\right.$ is the trace in spinor space only).

We fix m or $\sigma_{0}$ such that (74) compensates with the term $\int \sigma_{0+} \zeta=\int \sigma_{0} \zeta$ from (69), i.e.

$$
2 \mathrm{gN} \int \frac{\mathrm{m}}{\mathrm{p}^{2}+\mathrm{m}^{2}} \frac{\mathrm{d}^{2} \mathrm{p}}{(2 \pi)^{2}}=\sigma_{0}:=\frac{\mathrm{m}}{\mathrm{g}}
$$

Due to the fermion UV regularization u we find

$$
m=(1+\delta) e^{-\pi / \lambda} \quad, \quad \sigma_{0} \cong \sqrt{\lambda}^{-1} N^{1 / 2} e^{-\pi / \lambda}
$$

(remember that $\lambda \leq 1$ is fixed, and $\mathrm{g}^{2}=\frac{\lambda}{\mathrm{N}}$ ).

For a sharp cutoff at $\mathrm{p}^{2}=1$ we find for $1+\delta$ the value $\left(1-\mathrm{e}^{-\pi / \lambda}\right)^{-1 / 2}$. In our case $\delta$ cannot be calculated explicitly but easily estimated as $|\delta|<<1$ for $\lambda<1$. (76) shows that $\lambda>\pi$ does not make much sense in the cutoff theory as was mentioned in the introduction.

Doing the analogous calculation for the $\zeta_{-}$-terms we find that $(75)$ is also the condition for

$$
\operatorname{Tr} \frac{1}{p-m} g \zeta_{-}+\int \sigma_{0} \zeta_{-}=0
$$

Now we come to the terms quadratic in $\zeta_{ \pm}$: 
(78) $\frac{1}{2} \operatorname{Tr} F_{+}^{2}=\frac{1}{2} \operatorname{Tr}\left(\frac{1}{\not p+m} g \zeta_{+}\right)^{2}=\frac{\lambda}{2} \int \frac{d^{2} p}{(2 \pi)^{2}} \tilde{\zeta}_{+}^{*}(p) \operatorname{Tr}_{2}\left(\int \frac{d^{2} q}{(2 \pi)^{2}} \frac{1}{(\phi+m)(\not p+q+m)}\right) \tilde{\zeta}_{+}(p)$

$$
=\frac{1}{2}<\zeta_{+}, \pi \zeta_{+}>
$$

where $\pi$ denotes the fermion bubble

$$
\pi(p)=\lambda \operatorname{Tr}_{2} \int \frac{d^{2} q}{(2 \pi)^{2}} \frac{1}{(q+m)(p+q+m)}
$$

We then define

$$
\pi_{\text {ren }}(p):=\pi(p)-\pi(0)
$$

and

$$
\mu^{2}:=1+\pi(0)=1+2 \lambda \int \frac{d^{2} q}{(2 \pi)^{2}} \frac{-q^{2}+m^{2}}{\left(q^{2}+m^{2}\right)^{2}}
$$

The term 1 stems from the original ultralocal boson interaction which entered in $\mathrm{d} \mu_{\mathrm{f}}(\sigma)$.

The calculation for the $\zeta_{-}$term gives as in (78) a contribution

$$
\frac{1}{2}<\zeta_{-}, \pi \zeta_{-}>
$$

We will prove in Lemma 4 that

$$
\mu^{2}>0 \quad \text { and } \quad \mu^{2}+\pi_{\text {ren }}>0
$$

This tells us that $\pi_{\text {ren }}+\mu^{2}$ is a positive operator and thus may be legitimately absorbed in the covariance for $\zeta$, which we now define in a first attempt through its inverse

$$
\widetilde{\mathrm{C}}_{\mathrm{LS}}^{-1}={ }_{+}\left(\pi_{\mathrm{ren}}+\mu^{2}\right)_{+}+{ }_{-}\left(\pi_{\mathrm{ren}}+\mu^{2}\right)_{-}+\hat{\mathrm{f}}+\hat{\varepsilon}_{\gamma}
$$

where

$$
\varepsilon:=N^{-1}, \varepsilon_{\gamma}:=\varepsilon \chi_{\Lambda} \chi_{\gamma}, \hat{\varepsilon}_{\gamma}=\sqrt{\pi_{\text {ren }}+\mu^{2}} \varepsilon_{\gamma} \sqrt{\pi_{\text {ren }}+\mu^{2}}
$$


The indices LS indicate that $\widetilde{\mathrm{C}}^{-1}$ depends on the regions $\mathrm{L}, \mathrm{S}_{+}, \mathrm{S}_{-}$. The first two terms are those isolated above (78), (82). The $\varepsilon$ term ensures positivity of $\widetilde{\mathrm{C}}^{-1}$ also in the large field region $\mathrm{L} \subset \gamma$ (38), (43) ( $\hat{f}(\mathrm{p})$ vanishes for $\mathrm{p} \rightarrow 0)$.

Starting from $\widetilde{\mathrm{C}}_{\mathrm{LS}}^{-1}$ we will now define the final covariance by setting

$$
\mathrm{C}_{\gamma}^{-1}=\sqrt{\mu^{2}+\pi_{\text {ren }}}\left(1-\chi_{\gamma}+\varepsilon_{\gamma}+f\right) \sqrt{\mu^{2}+\pi_{\text {ren }}}
$$

The difference

$$
\widetilde{\mathrm{C}}_{\mathrm{LS}}^{-1}-\mathrm{C}_{\gamma}^{-1}
$$

contains a lot of terms which, however, are all easy to control. We find

$$
\begin{aligned}
& \widetilde{\mathrm{C}}_{\mathrm{LS}}^{-1}-\mathrm{C}_{\gamma}^{-1}=\sum_{\mathrm{i}=1}^{5} \mathrm{C}_{\mathrm{i}}, \mathrm{C}_{\mathrm{i}}=\mathrm{C}_{\mathrm{i}}(\gamma), \text { with } \\
& \mathrm{C}_{1}={ }_{+}\left(\sqrt{\mu^{2}+\pi_{\text {ren }}} \gamma \sqrt{\mu^{2}+\pi_{\text {ren }}}\right)++{ }_{-}\left(\sqrt{\mu^{2}+\pi_{\text {ren }}} \gamma \sqrt{\mu^{2}+\pi_{\text {ren }}}\right)- \\
& \mathrm{C}_{2}=-\left(\sqrt{\mu^{2}+\pi_{\text {ren }}} \hat{\gamma} \sqrt{\mu^{2}+\pi_{\text {ren }}}\right)+++\left(\sqrt{\mu^{2}+\pi_{\text {ren }}} \hat{\gamma} \sqrt{\mu^{2}+\pi_{\text {ren }}}\right)- \\
& \mathrm{C}_{3}={ }_{\mathrm{L}}\left(\sqrt{\mu^{2}+\pi_{\text {ren }}} \hat{\gamma} \sqrt{\mu^{2}+\pi_{\text {ren }}}\right) \mathrm{S}+\mathrm{s}\left(\sqrt{\mu^{2}+\pi_{\text {ren }}} \hat{\gamma} \sqrt{\mu^{2}+\pi_{\text {ren }}}\right) \mathrm{L} \\
& \mathrm{C}_{4}=\mathrm{L}\left(\sqrt{\mu^{2}+\pi_{\text {ren }}} \hat{\gamma} \sqrt{\mu^{2}+\pi_{\text {ren }}}\right)_{\mathrm{L}} \\
& \mathrm{C}_{5}=\hat{\Lambda}\left(\sqrt{\mu^{2}+\pi_{\text {ren }}} \hat{\gamma} \sqrt{\mu^{2}+\pi_{\text {ren }}}\right)_{\Lambda}+{ }_{\Lambda}\left(\sqrt{\mu^{2}+\pi_{\text {ren }}} \hat{\gamma} \sqrt{\mu^{2}+\pi_{\text {ren }}}\right)_{\Lambda} \\
& +\hat{\Lambda}\left(\sqrt{\mu^{2}+\pi_{\text {ren }}} \hat{\gamma} \sqrt{\mu^{2}+\pi_{\text {ren }}}\right) \hat{\Lambda}
\end{aligned}
$$

Here we set $+\left(\sqrt{\mu^{2}+\pi_{\text {ren }}}\right)_{\gamma}=\chi_{S_{+}}\left(\sqrt{\mu^{2}+\pi_{\text {ren }}}\right) \chi_{\gamma}$ etc. (see (43),(45)), and we put

$$
\delta_{L S}:=\sum_{i=2}^{4} C_{i}+\hat{\varepsilon}_{\gamma}
$$

We now write the expression for the two-point function using $\mathrm{d} \mu_{\gamma}(\zeta)$ defined as the Gaussian measure with mean zero and covariance $\mathrm{C}_{\gamma}$, normalized according to

$$
\int \mathrm{d} \mu_{\gamma}=1
$$


In writing $S_{2}$ we may dispose of a global normalization for $S_{2 u n}$ and $Z$. We choose it such that for $\gamma=\varnothing$ the normalization factor equals 1 . Then for $\gamma \neq \emptyset$ we get a factor $Z_{\gamma}$ due to the change of covariance from $C_{0}$ (for $\left.\gamma=\emptyset\right)$ to $C_{\gamma}$ which is ([5], Ch. 9)

$$
\mathrm{Z}_{\gamma}=\operatorname{Det}^{1 / 2}\left(\mathrm{C}_{\gamma} / \mathrm{C}_{0}\right)
$$

(We write Det instead of det since according to our rules det involves a flavour and spinor trace, which is absent here).

We would be satisfied if the attentive reader had become curious after all these preparations, what we are going to write for $S_{2}\left(f_{1}, f_{2}\right)$, the normalized two-point function :

we get

$$
\mathrm{S}_{2}\left(\mathrm{f}_{1}, \mathrm{f}_{2}\right)=\frac{\sum_{\ell, \mathrm{s}^{+}} \mathrm{Z}_{\gamma} \int \mathrm{d} \mu_{\gamma}(\zeta) \mathrm{F}_{2}(\zeta) \mathrm{G}_{\gamma}(\zeta)}{\sum_{\ell, \mathrm{s}^{+}} \mathrm{Z}_{\gamma} \int \mathrm{d} \mu_{\gamma}(\zeta) \mathrm{G}_{\gamma}(\zeta)}=\frac{\sum_{\ell, \mathrm{s}^{+}} \mathrm{Z}_{\gamma} \mathrm{I}_{\gamma}^{(2)}}{\sum_{\ell, \mathrm{s}^{+}} \mathrm{Z}_{\gamma} \mathrm{I}_{\gamma}^{(0)}}
$$

with

$$
F_{2}(\zeta)=\left\langle f_{1}, \frac{1}{p+g \sigma(\zeta)} f_{2}>\right.
$$

and

$$
\mathrm{G}_{\gamma}(\zeta)=\mathrm{G}_{1 \gamma}(\zeta) \mathrm{G}_{2 \gamma}^{\prime}(\zeta) \mathrm{G}_{3 \gamma}(\zeta) \mathrm{G}_{4 \gamma}^{\cdot}(\zeta) \mathrm{G}_{5 \gamma}(\zeta) \mathrm{G}_{6 \gamma}(\zeta)
$$

with

$$
\left.\mathrm{G}_{1 \gamma}=\mathrm{b}(\sigma) \prod_{\Delta \in \ell} \theta_{\Delta}^{\ell}(\sigma) \prod_{\Delta \in \mathrm{s}^{ \pm}} \theta_{\Delta}^{\mathrm{s}^{ \pm}}(\sigma) \mathrm{e}^{-1 / 2<\sigma, \mathrm{C}_{5} \sigma>} \mathrm{r}(\sigma) \quad \text { (where } \sigma=\sigma(\zeta)\right)
$$

(see (27), (47), (48)).

$$
G_{2 \gamma}^{\cdot}=\prod_{a} \operatorname{det}^{1 / 2}\left(1+A_{L a}\right) e^{-1 / 2<\sigma_{L}, \sigma_{L}>}
$$

(see (47), (53), (54)). 
The last term stems from the original ultralocal interactions. In $S_{ \pm}$the analogous contributions have been absorbed in the definition $\mu^{2}(81)$, (84) and thus in the measure $\mathrm{d} \mu_{\gamma}$ The relation between $\zeta_{\mathrm{L}}$ and $\sigma_{\mathrm{L}}$ (for which the large field condition was formulated) is in (68).

$$
\mathrm{G}_{5 \gamma}=\exp \left\{-\frac{1}{2}<\zeta, \delta_{\mathrm{LS}}, \zeta>-<\sigma_{02+}-\sigma_{02-}, \hat{\mathrm{f}} \zeta>-<\sigma_{02_{+}}-\sigma_{02-}, \hat{\mathrm{f}}\left(\sigma_{02+}-\sigma_{02-}\right)>\right\}
$$

(see (85)-(87),(69))

(97)

$$
G_{6 \gamma}=\exp \left\{-\left(\operatorname{Tr}\left(F_{+}^{2} F_{+}^{*}+F_{+} F_{+}^{* 2}+\frac{1}{2}\left(F_{+} F_{+}^{*}\right)^{2}+(+\leftrightarrow-)\right\} \operatorname{det}_{3}\left(1+K_{+}\right) \operatorname{det}_{3}\left(1+K_{-}\right)\right.\right.
$$

$(\operatorname{see}(52)-(54))$

We have thus separated $\mathrm{G}_{\gamma}$ into 6 parts. The first collects restrictions on $\sigma$ or $\zeta$, the second the essential part of the large field contributions. $G_{3 \gamma}$ contains the terms coupling the small and large field contributions. $G_{5 \gamma}$ contains terms which are small in $N$ (see Proposition 2). The second part of $\mathrm{G}_{4 \gamma}^{\prime}$ is a normalization term (note that the contributions of the form $<\sigma_{02+}, \sigma_{02-}>$ vanish due to (37), (55), (65) since dist $\left.\left(\mathrm{s}_{2+}, \mathrm{s}_{2-}\right) \geq 1\right) \cdot \mathrm{G}_{2 \gamma}^{\prime}, \mathrm{G}_{4 \gamma}^{\prime}$ carry a prime since their definition will be changed later (see (151),(152)). Finally $\mathrm{G}_{6 \gamma}$ contains the interaction terms in the small field region.

As a last preparation for the expansions we want to show that the covariance $C_{\gamma}(86)$ can be written as a sum over terms $C_{\gamma a}$ centered in the different connectivity components of $\gamma$. To do so we write the resolvent expansion for $\mathrm{C}_{\gamma}$

$$
\begin{aligned}
C_{\gamma} & =C_{0}+C_{0}\left(C_{0}^{-1}-C_{\gamma}^{-1}\right) C_{\gamma} \\
& =C_{0}+C_{0}\left(C_{0}^{-1}-C_{\gamma}^{-1}\right) C_{0}+C_{0}\left[\left(C_{0}^{-1}-C_{\gamma}^{-1}\right) C_{0}\right]^{2}+\ldots
\end{aligned}
$$

Using 


$$
\mathrm{C}_{0}^{-1}-\mathrm{C}_{\gamma}^{-1}=\sqrt{\mu^{2}+\pi_{\text {ren }}} \chi_{\gamma}(1-\varepsilon) \sqrt{\mu^{2}+\pi_{\text {ren }}}
$$

we deduce

$$
C_{\gamma}=\frac{1}{\sqrt{\mu^{2}+\pi_{\text {ren }}}} \frac{1}{\sqrt{1+f}} \sum_{m \geq 0}\left(\frac{1}{\sqrt{1+f}} \chi_{\gamma}(1-\varepsilon) \frac{1}{\sqrt{1+f}}\right)^{m} \frac{1}{\sqrt{1+f}} \frac{1}{\sqrt{\mu^{2}+\pi_{\text {ren }}}}
$$

Note that the expansion converges in norm and that the lowest order term equals $C_{0}=\frac{1}{\sqrt{\mu^{2}+\pi_{\text {ren }}}}$ $\frac{1}{1+\mathrm{f}} \frac{1}{\sqrt{\mu^{2}+\pi_{\text {ren }}}}$

Since $\left(\frac{1}{1+f}\right)(x, y) \equiv 0$ for $|x-y|>1$ and due to the definition of the connectivity components $\gamma_{a}$ of $\gamma(43)$ we find from (100)

$$
\mathrm{C}_{\gamma}=\mathrm{C}_{0}+\frac{1}{\sqrt{\mu^{2}+\pi_{\mathrm{ren}}}} \frac{1}{1+\mathrm{f}}\left(\sum_{\mathrm{a}, \mathrm{r} \geq 1} \chi_{\gamma \mathrm{a}}(1-\varepsilon)\left(\frac{1}{1+\mathrm{f}} \chi_{\gamma \mathrm{a}}(1-\varepsilon)\right)^{\mathrm{r}}\right) \frac{1}{1+\mathrm{f}} \frac{1}{\sqrt{\mu^{2}+\pi_{\mathrm{ren}}}}
$$

which we write as

$$
\mathrm{C}_{0}+\mathrm{C}^{(\gamma)}=\mathrm{C}_{0}+\frac{1}{\sqrt{\mu^{2}+\pi_{\mathrm{ren}}}} \widehat{C}_{\gamma} \frac{1}{\sqrt{\mu^{2}+\pi_{\mathrm{ren}}}}
$$

with $C^{(\gamma)}=\sum_{\mathbf{a}} C_{\mathbf{a}}^{(\gamma)}$

$$
\begin{aligned}
& \mathrm{C}_{\mathrm{a}}^{(\gamma)}:=\frac{1}{\sqrt{\mu^{2}+\pi_{\mathrm{ren}}}} \frac{1}{1+\mathrm{f}}\left(\sum_{\mathrm{r} \geq 1} \chi_{\gamma_{\mathrm{a}}}(1-\varepsilon)\left(\frac{1}{1+\mathrm{f}} \chi_{\gamma \mathrm{a}}(1-\varepsilon)\right)^{\mathrm{r}}\right) \frac{1}{1+\mathrm{f}} \frac{1}{\sqrt{\mu^{2}+\pi_{\mathrm{ren}}}} \\
& \widehat{\mathrm{C}}_{\gamma}:=\sum_{\mathrm{a}} \widehat{\mathrm{C}}_{\gamma \mathrm{a}}, \widehat{\mathrm{C}}_{\gamma \mathrm{a}}=\frac{1}{1+\mathrm{f}}\left(\sum_{\mathrm{r} \geq 1} \chi_{\gamma_{\mathrm{a}}}(1-\varepsilon)\left(\frac{1}{1+\mathrm{f}} \chi_{\gamma \mathrm{a}}(1-\varepsilon)\right)^{\mathrm{r}}\right) \frac{1}{1+\mathrm{f}}
\end{aligned}
$$

As a first application of this result we find that the normalization factor $Z_{\gamma}(88)$ factorizes over the connectivity components of $\gamma$

$$
\begin{aligned}
Z_{\gamma} & =\operatorname{Det}^{1 / 2}\left(\frac{C_{\gamma}}{C_{0}}\right)=\operatorname{Det}^{1 / 2}\left(1+\frac{1}{C_{0}} \sum_{a} C_{a}^{(\gamma)}\right) \\
& =\operatorname{Det}^{1 / 2}\left(1+\sum_{a, r \geq 1}\left((1-\varepsilon) \chi_{\gamma a} \frac{1}{1+f}\right)^{r}\right) \\
& =\prod_{a} \operatorname{Det}^{1 / 2}\left(1+\sum_{r \geq 1}\left((1-\varepsilon) \chi_{\gamma a} \frac{1}{1+f}\right)^{r}\right)
\end{aligned}
$$




$$
\left.=\prod_{\mathrm{a}} \operatorname{Det}^{1 / 2}\left(1+\frac{1}{\mathrm{C}_{0}} \mathrm{C}_{\mathrm{a}}^{(\gamma)}\right)\right)=\prod_{\mathrm{a}} \mathrm{Z}_{\gamma \mathrm{a}}
$$

(where we used the cyclicity of the determinant and the orthogonality of the $\chi_{\gamma_{\mathrm{a}}} \frac{1}{1+\mathrm{f}}$ ).

Now we bound $\mathrm{Z}_{\gamma \mathrm{a}}$

Lemma 3: $\quad 1 \leq \mathrm{Z}_{\gamma \mathrm{a}} \leq \mathrm{e}^{\mathrm{O}(1) \mathrm{N}^{1 / 10}\left|\gamma_{\mathrm{a}}\right|}$

Proof: $\quad \mathrm{Z}_{\gamma_{\mathrm{a}}}=\operatorname{Det}^{1 / 2}\left(1+\frac{(1-\varepsilon) \chi_{\gamma \mathrm{a}} \frac{1}{1+\mathrm{f}}}{1-(1-\varepsilon) \chi_{\gamma \mathrm{a}} \frac{1}{1+\mathrm{f}}}\right)$

$$
=\operatorname{Det}^{-1 / 2}\left(1-(1-\varepsilon) \chi_{\gamma a} \frac{1}{1+f}\right)
$$

$$
\begin{aligned}
& =\exp \left\{\left(-\frac{1}{2}\right) \operatorname{tr} \ln \left(1-(1-\varepsilon) \chi_{\gamma_{\mathrm{a}}} \frac{1}{1+\mathrm{f}}\right)\right\} \\
& =\exp \left\{\frac{1}{2} \operatorname{tr} \sum_{\mathrm{n} \geq 1} \frac{1}{\mathrm{n}}(1-\varepsilon)^{\mathrm{n}}\left(\frac{1}{1+\mathrm{f}} \chi_{\gamma_{\mathrm{a}}}\right)^{\mathrm{n}}\right\}
\end{aligned}
$$

(tr denotes a flavour and spinor singlet trace).

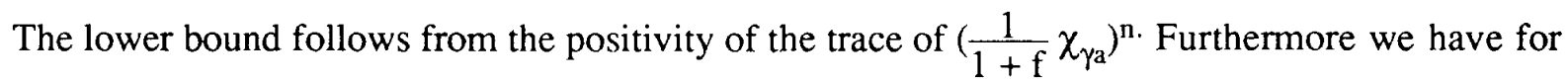
any positive Hermitian trace class operator $A$ and (orthogonal) projector $\mathrm{P}$ the following inequality

$$
\operatorname{Tr}(\mathrm{P} A \mathrm{P})^{\mathrm{n}} \leq \operatorname{Tr} \mathrm{P} \mathrm{A}^{\mathrm{n}} \mathrm{P}
$$

To see this evaluate the trace in an eigenbasis $\left\{\varphi_{i}\right\}$ of P A P with eigenvalues $\lambda_{i} \geq 0$. The inequality follows inductively from

$$
\operatorname{Tr} \mathrm{PA}^{\mathrm{r}}(\mathrm{PAP})^{\mathrm{s}} \leq \operatorname{Tr} \mathrm{PA}^{\mathrm{r}+1}(\mathrm{PA} \mathrm{P})^{\mathrm{s}-1}
$$

which in turn follows from

$$
\begin{aligned}
& \operatorname{Tr} \mathrm{PA}^{\mathrm{r}}(1-\mathrm{P}) \mathrm{A}(\mathrm{PAP})^{\mathrm{s}-1}=\sum_{\mathrm{i}} \lambda_{\mathrm{i}}^{\mathrm{s}-1}\left(\varphi_{\mathrm{i}}, \mathrm{A}^{\mathrm{r}}(1-\mathrm{P}) \mathrm{A} \varphi_{\mathrm{i}}\right) \\
& =\sum_{\mathrm{i}}\left[\lambda_{\mathrm{i}}^{\mathrm{s}-1}\left(\varphi_{\mathrm{i}}^{\mathrm{s}}, \mathrm{A}^{\mathrm{r}-1} \varphi_{\mathrm{i}}^{\mathrm{s}}\right)+\lambda_{\mathrm{i}}^{\mathrm{s}}\left(\varphi_{\mathrm{i}}, \mathrm{A}^{\mathrm{r}-1}(1-\mathrm{P}) \mathrm{A} \varphi_{\mathrm{i}}\right)\right] \geq 0,
\end{aligned}
$$


where we set $\varphi_{\mathrm{i}}^{\prime}=(1-\mathrm{P}) \mathrm{A} \varphi_{\mathrm{i}}$. Then the first term in the last sum is manifestly nonnegative and the second is so by induction on $r$.

Applying (105) to (104) we obtain

$$
\begin{aligned}
\operatorname{tr}\left(\frac{1}{1+f} \chi_{\gamma_{\mathrm{a}}}\right)^{\mathrm{n}} & \leq \operatorname{tr}\left(\chi_{\gamma_{\mathrm{a}}}\left(\frac{1}{1+\mathrm{f}}\right)^{\mathrm{n}} \chi_{\gamma_{\mathrm{a}}}\right) \\
& \leq \mathrm{O}(1) \frac{1}{2^{\mathrm{n}}}\left|\gamma_{\mathrm{a}}\right| \mathrm{N}^{1 / 10}
\end{aligned}
$$

The last inequality follows from the lower bound on $f(9),(63)$ and from

$$
\int d^{2} x\left(\frac{1}{1+x^{4}}\right)^{n} \sim \frac{1}{2^{n}}
$$

so that

(106) $\quad Z_{\gamma_{a}} \leq \exp \frac{1}{2} \sum_{n \geq 1} \frac{1}{n} \frac{(1-\varepsilon)^{n}}{2^{n}} O(1) N^{1 / 10}\left|\gamma_{a}\right| \leq e^{O(1) N^{1 / 10}\left|\gamma_{a}\right|}$

Remark: On inspection one finds that the constants may be arranged such that

(106') $\quad Z_{\gamma_{a}} \leq \mathrm{e}^{\mathrm{N}^{1 / 10}\left|\gamma_{\mathrm{a}}\right|}$ 


\section{ESTIMATES}

In this chapter we want to collect estimates which are independent of the cluster expansion techniques. They are required for the construction of the thermodynamic limit and for the proof of the exponential fall-off of the two-point function in the next chapter.

First we establish estimates on the fall-off of all non-local kernels and of the covariance. Then we present bounds on the Fredholm determinant and on the field variable in the small and large field regions. Using these bounds we show how to control the terms $G_{i \gamma}$ from (90).

\section{III.1. Fall-off properties of nonlocal kernels and consequences thereof.}

We start by establishing properties of $\pi(\mathrm{p})$ appearing in (79),... These properties imply that $\mathrm{C}_{\gamma}$ is positive and has exponential decay.

\section{Lemma 4 :}

(i) $\mu^{2}, \mu^{2}+\pi_{\text {ren }}$ (p) $>0$

(ii) The position space kernel of $\pi_{\text {ren }}(p)$, denoted $\pi_{\text {ren }}(x-y)$ decays as $\left|\pi_{\text {ren }}(\mathrm{x}-\mathrm{y})\right| \leq \mathrm{O}(1) \exp (-2 \mathrm{~m}|\mathrm{x}-\mathrm{y}|)$ Proof :

(i) For $\mu^{2}$ we find from (81), (75)

$$
\begin{gathered}
\mu^{2}=1+\pi(0)=1-2 \lambda \int \frac{\mathrm{d}^{2} \mathrm{q}}{(2 \pi)^{2}}\left(\frac{1}{\left(\mathrm{q}^{2}+\mathrm{m}^{2}\right)}-\frac{2 \mathrm{~m}^{2}}{\left(\mathrm{q}^{2}+\mathrm{m}^{2}\right)^{2}}\right) \\
=1+2 \lambda\left(-\frac{1}{2 \lambda}+\frac{1}{2 \pi}\left(1+\mathrm{O}\left(\mathrm{m}^{2}\right)\right)\right)=\frac{\lambda}{\pi}\left(1+\mathrm{O}\left(\mathrm{m}^{2}\right)\right)>0
\end{gathered}
$$

The correction term $\mathrm{O}\left(\mathrm{m}^{2}\right) \sim \mathrm{e}^{-2 \pi / \lambda} \ll 1 \mathrm{stems}$ from the fact that the propagators are UV regularized (see (5), (6)). Note that the first integral in (107) is exactly $\frac{1}{2 \lambda}$ (75).

Now we introduce the operator $\mathrm{V}$ with kernel

$$
V(x, y):=\int \frac{d^{2} q}{(2 \pi)^{2}} \frac{e^{i q(x-y)}}{\phi+m} \zeta(y), \quad \zeta(y) \in \mathfrak{L}^{2}(\Lambda)
$$

We find

$$
<\zeta,\left(\mu^{2}+\pi_{\mathrm{ren}}\right) \zeta>=\lambda \operatorname{Tr}_{2}\left(\mathrm{~V}^{2}+\mathrm{VV}^{*}\right)+\left(\mu^{2}-2 \mathrm{~m}^{2}\right)<\zeta, \zeta>
$$


Since $\mu^{2} \gg \mathrm{m}^{2}$ the second term is positive, and the first is nonnegative (note that $\operatorname{Tr}_{2} \mathrm{~V}^{2}$ is real since $\zeta$ is a real function), which proves

$$
\mu^{2}+\pi_{\text {ren }}>0
$$

(ii) $\quad \pi_{\text {ren }}(\mathrm{p})$ and thus also $\pi_{\mathrm{ren}}(\mathrm{x}-\mathrm{y})$ are in the Schwartz space $S\left(\mathbb{R}^{2}\right)$ due to the UV cutoff. Our specific choice of cutoff even assures analyticity of $\pi_{\text {ren }}(p)$ for

$$
(\operatorname{Imp})^{2} \leq 4 m^{2}\left(1+m^{2}+O\left(m^{4}\right)\right)
$$

where the correction terms come again from the cutoff.

This implies the assertion.

QED

\section{Lemma 5 :}

$$
0<\left(\frac{1}{p^{2}+m^{2}}\right)(x, y) \leq O(1) e^{-m|x-y|}
$$

$$
\begin{gathered}
\left|\left(\mu^{2}+\pi_{\text {ren }}\right)(x, y)\right| \leq O(1) e^{-2 m|x-y|},(x \neq y) \\
\left|\left(\mu^{2}+\pi_{\text {ren }}\right) \pm 1 / 2(x, y)\right| \leq O(1) e^{-\sqrt{2} m|x-y|},(x \neq y),
\end{gathered}
$$

(where again all propagators are regularized : $p \rightarrow p \mathrm{e}_{2}^{\frac{1}{2}} \mathrm{p}^{2}$ ).

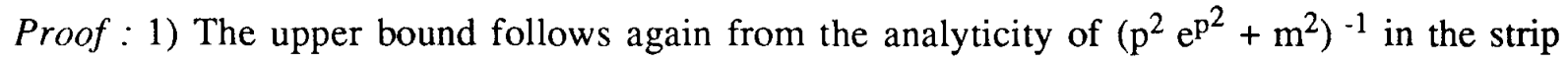

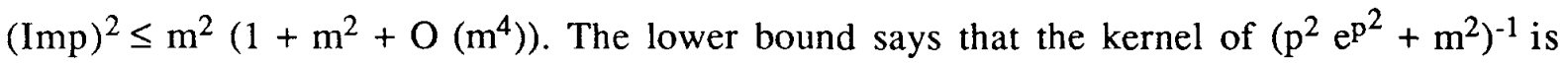
pointwise positive. To prove this we expand

$$
\frac{1}{p^{2} e^{2}+m^{2}}=e^{-p^{2}} \frac{1}{p^{2}+1-\left(1-m^{2} e^{-p^{2}}\right)}=e^{-p^{2}} \frac{1}{p^{2}+1} \sum_{n=0}^{\infty}\left(\frac{1}{p^{2}+1}-m^{2} \frac{1}{p^{2}+1} e^{-p^{2}}\right)^{n}
$$

$\frac{1}{\mathrm{p}^{2}+1}$ and $\mathrm{e}^{-\mathrm{p}^{2}}$ and thus also products of both have pointwise positive kernels in position space (for $\frac{1}{\mathrm{p}^{2}+1}$ note that

$$
\left.\int \mathrm{e}^{\mathrm{ipx}} \frac{1}{\mathrm{p}^{2}+1} \mathrm{~d}^{2} p=\int \mathrm{d}^{2} \mathrm{p} \mathrm{e}^{\mathrm{ipx}} \int_{0}^{\infty} \mathrm{dx} \mathrm{e}^{-\alpha\left(\mathrm{p}^{2}+1\right)}\right)
$$


Finally we may write

$$
\frac{1}{p^{2}+1}-m^{2} \frac{1}{p^{2}+1} e^{-p^{2}}=\frac{1}{p^{2}+1}\left(1-m^{2} e\right)+m^{2} e \int_{0}^{1} d s e^{-s\left(p^{2}+1\right)}
$$

Both terms have manifestly positive kernels in position space for $\mathrm{m}^{2} \mathrm{e}<1$ (which is assumed).

2) was proven in the previous lemma. The third statement follows from the following observations.

a) First regard the unregularized expression for $\pi_{\text {ren }}$ (which is given by a well-defined absolutely convergent integral). Using Feynman-parameters one gets

$$
\pi_{\text {ren,unreg }}(p)=\frac{\lambda}{4 \pi} \mathrm{p}^{2} \int_{0}^{1} \frac{1-4 \mathrm{x}(1-\mathrm{x})}{\mathrm{m}^{2}+\mathrm{p}^{2} \mathrm{x}(1-\mathrm{x})} \mathrm{dx}
$$

From this expression one reads off

$$
\operatorname{Re} \pi_{\text {ren,unreg }}(p) \geq \operatorname{Re} \pi_{\text {ren,unreg }}\left(i_{2}\right)\left(\text { for } p=p_{1}+i p_{2}, p_{2}=\operatorname{Im} p\right.
$$

And for $\left|p_{2}\right| \leq \sqrt{2} m$ we find

$$
\mathrm{R}_{\mathrm{e}} \pi_{\text {ren,unreg }}\left(\mathrm{ip}_{2}\right) \leq-\frac{\lambda}{3 \pi}
$$

From these statements we find that ${\sqrt{\mu^{2}+\pi_{\text {ren,unreg }}}}_{1}$ is analytic for $\left|p_{2}\right| \leq \sqrt{2} \mathrm{~m}$, since $\mu^{2}=\frac{\lambda}{\pi}\left(1+\mathrm{O}\left(\mathrm{m}^{2}\right)\right)$.

b) Now since

(116) $\pi_{\text {ren }}(p)=\pi_{\text {ren,unreg }}(p)\left(1+O\left(m^{2}\right)\right)$,

due to the quadratic convergence of the renormalized integral we obtain for $\mid \operatorname{Impl} \leq \sqrt{2} \mathrm{~m}$

$$
\operatorname{Re} \pi_{\text {ren }}(p) \geq \frac{\lambda}{3 \pi}\left(1+O\left(m^{2}\right)\right)
$$

and therefore statement 3 ) (where, of course, $\sqrt{2} \mathrm{~m}$ is not optimal). We omit the tedious estimation of the coefficient in $\mathrm{O}\left(\mathrm{m}^{2}\right)$. 
Using the fall-off properties established in Lemma 3, Lemma 4 we can now prove estimates on the terms in $\delta_{\mathrm{LS}}$ from $\mathrm{G}_{5 \gamma}(96),(87)$.

Lemma 6 : For the operators $C_{i}$ with kernels $C_{i}(x, y), i=1, \ldots, 5$ defined in (87) we have the following estimates

(i) $C_{1} \geq 0$ as operator on $\mathfrak{L}^{2}\left(\mathbb{R}^{2}\right)$

(ii) $\left|C_{j}(x, y)\right| \leq O(1) \inf \left(e^{-\sqrt{2} m|x-y|}, N^{-\sqrt{2}}\right) \quad j=2,3,4$

(iii) $\left\|C_{5}\right\| \leq \mathrm{O}(1)$

\section{Proof :}

(i) follows from the positivity of $\mu^{2}+\pi_{\text {ren }}$ (Lemma 4)

(ii) follows from Lemma 5 and the definitions (35)-(43) which imply (for $\mathrm{C}_{3}, \mathrm{C}_{4}$ ) $\operatorname{dist}(\hat{\gamma}, L)>\frac{M}{2}-2($ see $(43))$

For $C_{2}$ we proceed as follows : If $x \in S_{+}$and $y \in S_{\text {. }}$ (or vice versa) we have either dist $(x, y) \geq \frac{M}{2}$ or dist $(x, y)<\frac{M}{2}$. In the second case there exists some $\Delta \in \mathrm{l}_{1}$ such that dist $(\mathrm{x}, \Delta)$ $+\operatorname{dist}(y, \Delta) \leq \frac{M}{2}(\operatorname{see}(36)-(38))$.

This implies

$$
\operatorname{dist}(\mathrm{x}, \hat{\gamma})+\operatorname{dist}(\mathrm{y}, \hat{\gamma}) \geq \frac{\mathrm{M}}{2}
$$

Collecting the distance factors in $C_{2}-C_{4}$ we obtain (118) using $M=\frac{2}{m} \log N$.

(iii) is trivial.

Lemma 7 : For the covariance $C_{\gamma}=C_{0}+C^{(\gamma)}(101)$ we have the following estimates : For $\gamma \neq \varnothing$

$$
\left|C^{(\gamma)}(x, y)\right| \leq O\left(\frac{1}{m^{2}}\right) N^{1 / 10} \exp ^{\{-\sqrt{2} m(\operatorname{dist}(x, \gamma)+\operatorname{dist}(y, \gamma))\}}
$$

If $\mathrm{x}, \mathrm{y} \in \Gamma^{\prime}$ or $\mathrm{x} \in \Gamma_{\mathrm{a}}, \mathrm{y} \in \Gamma_{\mathrm{b}}, \mathrm{a} \neq \mathrm{b}$ we find $\left|\mathrm{C}^{(}(\mathrm{x})(\mathrm{x}, \mathrm{y})\right| \leq \mathrm{O}\left(\mathrm{N}^{-2}\right)$

If $\mathrm{x} \in \Gamma, \mathrm{y} \in \Gamma^{\prime}:\left|C^{(\gamma)}(\mathrm{x}, \mathrm{y})\right| \leq \mathrm{O}\left(\mathrm{N}^{-1}\right)$

$$
\left|C_{0}(x, y)\right| \leq O(1) e^{-\sqrt{2} m|x-y|}
$$


Proof : (120) follows from Lemmas 5,4 and from the properties of $f(63)$ which imply that $\widehat{C}_{\gamma}$ is supported in $\{(\mathrm{x}, \mathrm{y}) \mid$ dist $(\mathrm{x}, \gamma)$, dist $(\mathrm{y}, \gamma) \leq 1\}$ and from a calculation similar to that in the proof of Lemma 3. Let $\Delta_{1}, \Delta_{2}$ be two squares in $\gamma$ with characteristic functions $\chi_{1}, \chi_{2}$. Then

$$
\mathrm{k} \chi_{1}, \widehat{\mathrm{C}}_{\gamma} \chi_{2}>1 \leq<\chi_{1}, \widehat{\mathrm{C}}_{\gamma} \chi_{1}>^{1 / 2}<\chi_{2}, \widehat{\mathrm{C}}_{\gamma} \chi_{2}>^{1 / 2}
$$

and

$$
<\chi_{\mathrm{i}}, \widehat{\mathrm{C}}_{\gamma} \chi_{\mathrm{i}}>\leq \mathrm{O}(1) \sum_{\mathrm{n}}(1-\varepsilon)^{\mathrm{n}} \operatorname{Tr}\left(\frac{1}{1+\mathrm{f}}\right)^{\mathrm{n}} \leq \mathrm{O}(1) \mathrm{N}^{1 / 10} \sum_{\mathrm{n}}\left(\frac{1}{2}\right)^{\mathrm{n}}
$$

On summing over the squares from $\gamma$ and using the fall-off of $\sqrt{\mu_{+}^{2}+\pi_{\text {ren }}}$ this leads to (120). The subsequent statements follow from the fall-off of $\left(\sqrt{\mu^{2}+\pi_{\text {ren }}}\right)^{-1}$ and the definitions of $\gamma, \Gamma$. (121) follows using Lemma 5 and the properties of $f(9)$, (63).

QED

\section{III.2. Estimates on the Fredholm determinant in the small and large field blocks}

III.2.1. The large field region

A large field square (lfs) $\Delta$ was defined in (30), (31) to be one carrying a factor

$$
\theta\left(\frac{\left\|A_{\Delta}\right\|_{2}}{N^{\alpha}}-1\right)
$$

which vanishes unless

(122) $\left\|A_{\Delta}\right\|_{2}>\frac{3}{4} N^{\alpha}, \quad \alpha=\frac{1}{10}$

$\left\|A_{\Delta}\right\|_{2}$ was defined as the HS norm of the operator $A_{\Delta}$. Let $U$ be a union of squares $\Delta$. Define $\left\|A_{U}\right\|_{2}, A_{U}$ as $\left\|A_{\Delta}\right\|_{2}, A_{\Delta}$.

Lemma 8: $\ln \operatorname{det}\left(1+A_{U}\right) \leq \operatorname{Tr} A_{U}-\frac{1}{2} \operatorname{Tr} \frac{A_{U}^{2}}{2+A_{U}} \leq \operatorname{Tr} A_{U}$

Proof: Since $\mathrm{A}_{\mathrm{U}}$ is traceclass, selfadjoint and > - 1, the last inequality is true and we may write 


$$
\ln \operatorname{det}\left(1+A_{U}\right)=\operatorname{Tr} \ln \left(1+A_{U}\right)=\sum_{i=1}^{\infty} \ln \left(1+\lambda_{i}\right)
$$

where $\lambda_{i}=\lambda_{i}\left(\sigma_{U}\right)$ are the eingenvalues of $A_{U} \lambda_{1} \geq \lambda_{2} \geq \ldots$ (which are (are least) $2 \mathrm{~N}$-fold degenerate). Note that $\sigma_{U}$ as usual may be assumed to be in $\mathfrak{L}^{2}(U)$. Then the assertion follows from $\ln (1+\mathrm{x}) \leq \mathrm{x}-\frac{1}{2} \frac{\mathrm{x}^{2}}{2+\mathrm{x}^{2}}$ for $\mathrm{x}>-1$.

QED

Now we regard $U=\ell(30)$, (35).

Lemma 9 : If $\left\|\mathrm{A}_{\Delta}\right\|_{2}>\frac{3}{4} \frac{1}{\mathrm{~N} 10} \forall \Delta \subset \ell$, we have $\operatorname{Tr} \frac{\mathrm{A}_{\ell}^{2}}{2+\mathrm{A}_{\ell}} \geq \frac{1}{4}|\ell| \mathrm{N}^{1 / 5}$

Proof: To evaluate the trace we choose the following complete orthonormal system : In any square $\Delta$ we choose a complete system of orthonormal eigenfunctions of $A_{\Delta}$ with eigenvalues $\lambda_{\text {is }}\left(\sigma_{\Delta}\right)$.

We find

$$
\begin{gathered}
\operatorname{Tr} \frac{A_{\ell}^{2}}{2+A_{\ell}}=\sum_{\Delta, i} \frac{\lambda_{i \Delta}^{2}}{2+\lambda_{i \Delta}}=\sum\left(\sum_{\lambda_{i \Delta}>\frac{1}{10}} \frac{\lambda_{i \Delta}^{2}}{2+\lambda_{i \Delta}}+\sum_{\lambda_{i} \leq \leq \frac{1}{10}}^{\sum} \frac{\lambda_{i \Delta}^{2}}{2+\lambda_{i \Delta}}\right) \\
\quad \geq \sum_{\Delta}\left(\sum_{>\frac{1}{10}} \frac{\lambda_{i \Delta}}{21}+\frac{10}{21} \sum_{\leq \frac{1}{10}} \lambda_{i \Delta}^{2}\right) \geq \frac{10}{21} \frac{9}{16}|\ell| N^{1 / 5} .
\end{gathered}
$$

The last inequality is true for a single $\Delta$, if the first sum in the brackets does not vanish, due to the $2 \mathrm{~N}$ fold degeneracy. If it vanishes, the second sum fulfills this bound due to (122).

QED

At a late stage of our estimations we shall also require a distinction between the lfs. A lfs is called a very large field square (vlfs) if

$$
<\varphi_{\Delta}, \mathrm{A}_{\Delta} \varphi_{\Delta}>>1
$$

where for given $\sigma_{\Delta}$ we define

$$
\varphi_{\Delta}\left(\sigma_{\Delta}\right)=\frac{\sigma_{\Delta}}{\left\|\sigma_{\Delta}\right\|} \quad\left(\left\|\sigma_{\Delta}\right\|=\left(\int \sigma^{2} d^{2} x\right)^{1 / 2}\right)
$$

Lemma 10 : For any set $\mathrm{V}$ of vlfs $\Delta$ we have 
(128)

$$
\frac{1}{2} \operatorname{Tr} \frac{A_{V}^{2}}{2+A_{V}} \geq \frac{3}{50} N|V|+\frac{1}{20} \int_{V} \sigma^{2}(x) d^{2} x .
$$

If $\Delta \in \ell / V$ we have

$$
\mathrm{g}^{2} \int_{\Delta} \sigma^{2} \leq 4 \lambda\left(1+\frac{\mathrm{m}^{2}}{2 \lambda}\right)
$$

Proof: As in the proof of Lemma 9 we have

$$
\operatorname{Tr} \frac{A_{V}^{2}}{2+A_{V}} \geq \frac{3}{7} \sum_{\Delta \in V} \sum_{i}^{(1)}<\varphi_{i \Delta}, A_{\Delta} \varphi_{i \Delta}>
$$

where $\Sigma_{i}^{(1)}$ is over a normalized basis $\varphi_{i \Delta}$ of that subspace of $\mathfrak{L}^{2}(\Delta)$ where $A_{\Delta} \geq \frac{1}{3}$. By assumption then

$$
\sum_{i}^{(1)}<\varphi_{i \Delta}, A_{\Delta} \varphi_{i \Delta}>\geq 2 N<\varphi_{\Delta}^{(1)}, A_{\Delta} \varphi_{\Delta}^{(1)}>\geq 2 N\left(<\varphi_{\Delta}, A_{\Delta} \varphi_{\Delta}>-\frac{1}{3}\right)
$$

where $\varphi_{\Delta}^{(1)}$ is the projection of $\varphi_{\Delta}$ onto that subspace. Now

$$
\begin{gathered}
<\varphi_{\Delta}, A \varphi_{\Delta}>-\frac{1}{3}=2 \cdot \frac{1}{2}<\varphi_{\Delta}, A \varphi_{\Delta}>-\frac{1}{3} \geq \\
\frac{1}{2}+\frac{1}{2} \frac{1}{\left\|\sigma_{\Delta}\right\|^{2}} \int_{\Delta x \Delta}\left\{g^{2} \sigma^{2}(x) F(x-y) \sigma^{2}(y)-m^{2} \sigma(x) F(x-y) \sigma(y)\right\}-\frac{1}{3} \\
\geq\left(\frac{1}{2}-\frac{1}{3}-\frac{m^{2}}{4 \lambda}\right)+\frac{1}{2} \frac{1}{\left\|\sigma_{\Delta}\right\|^{2}} \int_{\Delta x \Delta}\left(g^{2} \sigma^{2}(x) F(x-y) \sigma^{2}(y)\right) \\
\geq\left(\frac{1}{6}-\frac{m^{2}}{4 \lambda}\right)+\frac{1}{8 N}\left(\int_{\Delta}\right)
\end{gathered}
$$

with the following explanations : we set $F(x-y)=\int \frac{e^{i p(x-y)}}{p^{2} e^{p 2}+m^{2}} \frac{d^{2} p}{(2 \pi)^{2}}$.

$F(x)$ is smooth, $F(0)=\frac{1}{2 \lambda}(75)$ and $F(x) \geq \frac{1}{4 \lambda}$ for $|x| \leq \sqrt{2}$, since $m^{2}<<1$. 
Furthermore

$$
\int_{\Delta x \Delta} \sigma^{2} F(x-y) \sigma^{2} \geq \frac{1}{4 \lambda}\left(\int \sigma^{2}\right)^{2}
$$

From this one directly verifies (128) for $\frac{1}{6}-\frac{m^{2}}{4 \lambda}>\frac{1}{7}$ (which we assume). The same estimates as used previously then show that $<\varphi_{\Delta}, A \varphi_{\Delta}><1$ implies

$$
\frac{1}{4 \lambda} \mathrm{g}^{2}\left(\int_{\Delta} \sigma^{2}\right) \leq 1+\frac{\mathrm{m}^{2}}{2 \lambda}
$$

which is (129).

From Lemma 8, 9, 10 we find directly

Lemma 11 : (i) For a set of lfs $\ell$ we have

(135) $\operatorname{det}\left(1+A_{1}\right) \leq \exp \left(\operatorname{Tr} A_{1}-\frac{1}{8}|\ell| N^{1 / 5}\right)$

(ii) For a set of vlfs $V$ we have

(136) $\operatorname{det}\left(1+A_{V}\right) \leq \exp \left(\operatorname{Tr} A_{V}-\frac{3}{50}|V| N-\frac{1}{20} \int_{V} \sigma^{2}(x) d^{2} x\right)$

\section{III.2.2. The small field region}

$\Delta$ is a small field square (sfs) if it carries a factor $1-\theta\left(\frac{\left\|A_{\Delta}\right\|_{2}}{N^{\alpha}}-1\right)$, which vanishes unless

$$
\left\|\mathrm{A}_{\Delta}\right\|_{2}<\frac{5}{4} \mathrm{~N}^{1 / 10}
$$

We want to show that this condition implies bounds on the field variable itself if integrated over small regions. First we prove

Lemma 12 : For a sfs $\Delta$ with $\int_{\Delta} \sigma>0$ we have

$$
O<\mathrm{g} \int_{\Delta^{\cdot}} \sigma \leq \mathrm{g} \int_{\Delta^{\prime}}|\sigma<\sqrt{2} \mathrm{~m}| \Delta^{\prime} \mid
$$


for any rectangle $\Delta^{\prime} \subset \Delta$ with $\left|\Delta^{\prime}\right| \geq \delta, 0<\delta<1$ suitably chosen, and the corresponding statement for $\int_{\Delta^{\prime}} \sigma<0$

Proof : The proof is by contradiction

( $\alpha$ ) Assume $\mathrm{g} \int_{\Delta^{\prime}} \sigma \geq \sqrt{2} \mathrm{~m}\left|\Delta^{\prime}\right|$ for some suitable $\Delta^{\prime}$. Then with $\Delta_{+}=\left\{\mathrm{x} \in \Delta^{\prime} \mid \sigma(\mathrm{x}) \geq 0\right\}$, $\Delta_{-}=\Delta^{\prime} \backslash \Delta_{+}$we find for

$$
\varphi_{+}:=\frac{1}{\left|\Delta_{+}\right|^{1 / 2}} \chi_{\Delta+}
$$

(140) $\left|<\varphi_{+} A \varphi_{+}>1 \geq \frac{1}{\left|\Delta_{+}\right|} \frac{1}{4 \lambda}\left(\int_{\Delta_{+}} \mathrm{g} \sigma_{+}\right)^{2}-\frac{\mathrm{m}^{2}}{2 \lambda}\right| \Delta_{+}\left|\geq \frac{1}{4 \lambda\left|\Delta_{+}\right|}\left(\sqrt{2} \mathrm{~m}\left|\Delta^{\prime}\right|\right)^{2}-\frac{\mathrm{m}^{2}}{2 \lambda}\right| \Delta^{\prime} \mid \geq \frac{1}{3} \frac{\mathrm{m}}{\lambda}$

(see $(132),,(133)$ for the first inequality and note $m<<1)$

so that

$$
\left\|\mathrm{A}_{\Delta}\right\|_{2}^{2} \geq 2 \mathrm{~N}\left(\frac{1}{3} \frac{\mathrm{m}}{\lambda}\right)^{2}
$$

which contradicts (137) for $\mathrm{N}^{4 / 5} \geq 8 \frac{\lambda^{2}}{\mathrm{~m}^{2}}$ (which we assume). The bound on $\int|\sigma|$ is proven analogously by changing $\varphi_{+} \rightarrow \frac{\operatorname{sign} \sigma}{\left|\Delta^{\prime}\right|^{1 / 2}} \chi_{\Delta^{\prime}}$.

( $\beta$ ) assume $\int_{\Delta^{\prime}} \sigma \leq 0$ for some suitable $\Delta^{\prime}$. Since $\int_{\Delta} \sigma>0$ there exists some rectangle $\Delta^{\prime \prime} \subset \Delta$, $\Delta^{\prime} \subset \Delta^{\prime \prime}$ such that $\int_{\Delta^{\prime \prime}} \sigma=0$; and we can find (possibly new) squares $\Delta^{\prime}, \Delta^{\prime \prime}$ with arbitrarily small sidelengths such that all relations still hold. Calling $\varphi:=\frac{1}{\left|\Delta^{\prime \prime}\right|^{1 / 2}} \chi_{\Delta^{\prime \prime}}$ we estimate

$$
|<\varphi, A \varphi>| \geq \mathrm{m}^{2} \frac{1}{2 \lambda}\left(1-|\Delta|^{\prime \prime}\right)|\Delta|^{\prime \prime}-\mathrm{g}^{2}\left(\int_{\Delta^{\prime \prime}}|\sigma|^{2}\right) \geq\left|\Delta^{\prime \prime}\right| \frac{\mathrm{m}^{2}}{2 \lambda}\left(1-3\left|\Delta^{\prime \prime}\right|\right) .
$$

In the first inequality we used 


$$
\begin{gathered}
\int_{\Delta^{\prime \prime} \mathrm{x} \Delta^{\prime \prime}} \sigma(\mathrm{x}) \mathrm{F}(\mathrm{x}-\mathrm{y}) \sigma(\mathrm{y})=\int_{\Delta^{\prime \prime} \Delta^{\prime \prime}} \sigma(\mathrm{x})(\mathrm{F}(\mathrm{x}-\mathrm{y})-\mathrm{F}(0)) \sigma(\mathrm{y}) \leq \sup _{\mathrm{x}, \mathrm{y} \in \Delta^{\prime \prime}}|\mathrm{F}(\mathrm{x}-\mathrm{y})-\mathrm{F}(0)|\left(\int_{\Delta^{\prime \prime}}|\sigma|\right)^{2} \\
\leq\left|\Delta^{\prime \prime}\right|\left(\int_{\Delta^{\prime \prime}}|\sigma|\right)^{2}
\end{gathered}
$$

using that $|F(x-y)-F(0)| \leq\left|\Delta^{\prime \prime}\right|$ for $x, y \in \Delta^{\prime \prime}$ and $\left|\Delta^{\prime \prime}\right|<\frac{1}{4}$ (which in turn follows by inspection of F). This again contradicts (137) if

$$
\left|\Delta^{\prime \prime}\right| \frac{m^{2}}{2 \lambda}\left(1-3\left|\Delta^{\prime \prime}\right|\right)(2 N)^{1 / 2}>\frac{5}{4} N^{1 / 10}
$$

which we assume to be true for $\frac{1}{5}>\left|\Delta^{\prime \prime}\right| \geq \delta>0$.

QED

With the aid of Lemma 12 we can show now that the mean of the translated field variable $\zeta$ in a sfs is small when multiplied by $\mathrm{g}$.

Lemma 13 : For a sfs $\Delta$ with $\int_{\Delta} \sigma>0$ we find

$$
\begin{aligned}
\mathrm{g} \int_{\Delta}|\zeta| \leq & \frac{15}{2} \frac{\lambda}{\mathrm{m}} \mathrm{N}^{-\frac{2}{5}}, \quad \text { where } \mathrm{g} \zeta=\mathrm{g} \sigma-\mathrm{m} \\
& \mathrm{g}^{2} \int_{\Delta} \zeta^{2} \leq \mathrm{O}\left(\frac{1}{\mathrm{~m}^{2}}\right) \mathrm{N}^{-4 / 5}
\end{aligned}
$$

(similarly for $\int_{\Delta} \sigma<0$ and $\mathrm{g} \zeta=\mathrm{g} \sigma+\mathrm{m}$ )

Proof: If $\mathrm{g} \int_{\Delta}|\zeta|>\frac{15}{2} \frac{\lambda}{\mathrm{m}} \mathrm{N}^{-\frac{2}{5}}=\mathrm{a} \quad$ then either

$$
\text { (i) } \mathrm{g} \int_{\Delta} \zeta_{+}>\frac{1}{2} \mathrm{a} \text { or (ii) } \mathrm{g} \int_{\Delta} \zeta_{-}>\frac{1}{2} \mathrm{a}
$$

where $\zeta_{( \pm)}=\sup (0,( \pm) \zeta(\mathrm{x}))$. Set also $\Delta_{+}=\{\mathrm{x} \in \Delta \mathrm{I} \zeta(\mathrm{x})>0\}, \Delta_{-}=\Delta \Delta_{+}$.

We assume (ii) to be true ((i) is simpler). We have

$$
\left\|A_{\Delta}\right\|_{2}=\left\|\left[g \zeta \frac{1}{p^{2}+m^{2}}(g \zeta+m)\right]_{\Delta}\right\|_{2}
$$

Choosing $\varphi_{-}=\frac{-\chi\left(\Delta_{-}\right)}{\left|\Delta_{-}\right|^{1 / 2}}$ we obtain from (137) (see (132) pp.) 


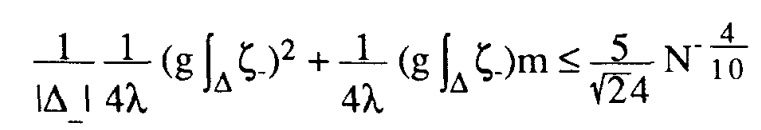

This implies

$$
\left(\mathrm{g} \int_{\Delta} \zeta_{-}\right) \leq \frac{5}{\sqrt{2}} \frac{\lambda}{\mathrm{m}} \mathrm{N}^{-\frac{2}{5}}
$$

which contradicts (ii) and thus proves (144), first part. The second inequality is proven similarly on replacing $\varphi_{-}$by $\frac{\zeta_{\Delta}}{\left\|\zeta_{\Delta}\right\|}$.

Then we obtain instead of (146)

$$
\frac{\mathrm{g}^{2}}{4 \lambda}\left\|\zeta_{\Delta}\right\|^{2}+\frac{\mathrm{g}}{4 \lambda} \mathrm{m}\left\|\zeta_{\Delta}\right\| \leq \mathrm{N}^{-4 / 10}
$$

which implies

or

$$
\mathrm{g}\left\|\zeta_{\Delta}\right\| \leq \mathrm{O}\left(\frac{1}{\mathrm{~m}}\right) \mathrm{N}^{-4 / 10}
$$

$$
\mathrm{g}^{2}\left(\int_{\Delta} \zeta^{2}\right) \leq \mathrm{O}\left(\frac{1}{\mathrm{~m}^{2}}\right) \mathrm{N}^{-4 / 5}
$$

Now let $\Delta_{+}$and and $\Delta_{\text {. }}$ be two small field squares (sfs) with a common edge belonging to $\mathrm{s}_{+}$ and s. (35) respectively. (33) then implies that there exists a square $\Delta \subset \Delta_{+} \cup \Delta_{-}$such that

$$
\int_{\Delta} \sigma=0
$$

(since $\int_{\Delta(\mathrm{s})} \sigma$ changes continuously from negative into positive values, if we move continuously $\Delta(\mathrm{s})$ from $\Delta_{-}$into $\left.\Delta_{+}\left(\Delta(0)=\Delta_{-}, \Delta(1)=\Delta_{+}\right)\right)$. Now

\section{Lemma 14 :}

$$
-\frac{1}{2} \leq \int_{\Delta} \sigma \leq \frac{1}{2} \Rightarrow\left\|\mathrm{A}_{\Delta}\right\|_{2} \geq \frac{5}{4} \mathrm{~N}^{\alpha}
$$

Proof : 
The proof is identical to the reasoning in the proof of Lemma $12(\beta)$ : one shows (142) (with a small correction $\sim \mathrm{N}^{-1 / 2}$ if $\int_{\Delta} \sigma \neq 0$ ) to be true for a subsquare $\Delta^{\prime}$ of $\Delta$ which implies the statement.

QED

Taking together Lemma $8,9,14$ we easily find for two squares $\Delta_{+}, \Delta_{-}$as above, i.e. belonging to $\ell_{1} \backslash \ell$

$$
\operatorname{det}\left(1+\mathrm{A}_{\Delta_{+} \cup \Delta_{-}}\right) \leq \operatorname{Tr} \mathrm{A}_{\Delta_{+} \cup \Delta_{-}}-\frac{1}{4} \mathrm{~N}^{1 / 5}
$$

\section{III.3. Bounds on the interaction terms in the functional integral}

We bound the interaction terms present in (90). First we perform a change of normalization. As can be seen in (95) the interaction in the small field region is normalized such that it does not vanish for $\zeta \equiv 0$, but is of order $-\mathrm{N}\left(\left|\mathrm{S}_{+}\right|+\left|\mathrm{S}_{-}\right|\right)$. Our presentation, hinging on the fact that the large field region is suppressed in probability, would thus go completely astray if such a term were not present in $\mathrm{L}$. But we have

\section{Lemma 15 :}

$$
\mathrm{e}_{2}^{\frac{1}{2}} \operatorname{Tr} \mathrm{A}_{\mathrm{L}}-\frac{1}{2}<\sigma_{\mathrm{L}}, \sigma_{\mathrm{L}}>\mathrm{e}^{-\frac{1}{2}<\sigma_{0 \mathrm{~L}}, \sigma_{0 \mathrm{~L}}>}
$$

Remark : (151) shows that exactly the same normalization factor as in (95) is also present per large field square. It just drops out if we divide numerator and denominator in (90) by this factor so that we may redefine

$$
\begin{gathered}
\mathrm{G}_{2 \gamma}=\Pi_{\mathrm{a}} \operatorname{det}_{2}^{1 / 2}\left(1+\mathrm{A}_{\mathrm{La}}\right) \\
\mathrm{G}_{4 \gamma}=\exp \left(-\frac{1}{2}<\zeta, \mathrm{C}_{1} \zeta>\right) \\
\text { (note that } \operatorname{Tr} \mathrm{A}_{\mathrm{L}}=\Pi_{\mathrm{a}} \operatorname{Tr} \mathrm{A}_{\mathrm{La}} \text { ) }
\end{gathered}
$$

and we use henceforth $\mathrm{G}_{2 \gamma}, \mathrm{G}_{4 \gamma}$ instead of $\mathrm{G}_{2 \gamma}^{\prime}, \mathrm{G}_{4 \gamma}^{\prime}$.

Proof :

$$
\begin{gathered}
\operatorname{Tr} A_{L}=2 N \int\left(g^{2} \sigma_{L}(x) F(0) \sigma_{L}(x)-m^{2} \chi_{L}(x) F(0)\right) d^{2} x \\
=\frac{2 N g^{2}}{2 \lambda}<\sigma_{L}, \sigma_{L}>-2 N \frac{m^{2}}{2 \lambda}|L| \\
=<\sigma_{L}, \sigma_{L}>-\sigma_{0}^{2}|L|
\end{gathered}
$$

Now we bound $G_{3 \gamma}$ : 


\section{Lemma 16 :}

$$
\begin{aligned}
& \operatorname{det}(1+Q)=\operatorname{det}\left(1+Q^{*}\right)=\operatorname{det}^{1 / 2}\left(1+Q+Q^{*}+Q^{*}\right) \\
& 0<\operatorname{det}(1+Q) \leq 1
\end{aligned}
$$

Proof: $\mathrm{Q}=\frac{1}{1+\widehat{\mathrm{K}}} \mathrm{K}^{\prime}$, where $\widehat{\mathrm{K}}=\sum_{\mathrm{a}} \mathrm{A}_{\mathrm{La}}+\mathrm{K}_{+}+\mathrm{K}_{-}$(see (55)).

By cyclicity we find

$$
\operatorname{det}(1+Q)=\operatorname{det}\left(1+Q^{*}\right), \text { noting that } Q^{*}=K^{\prime} \frac{1}{1+\widehat{K}}
$$

so that

$$
\operatorname{det}^{2}(1+Q)=\operatorname{det}\left(1+Q+Q^{*}+Q^{*}\right)
$$

Since $\operatorname{Tr} Q^{2 n+1}=0$, we also find

$$
\operatorname{det}(1+Q)=\operatorname{det}^{1 / 2}\left(1-Q^{2}\right)=\operatorname{det}^{1 / 2}(1-\widetilde{Q})
$$

with

$$
\widetilde{Q}=\frac{1}{\sqrt{1+\widehat{K}}} K^{\prime} \frac{1}{1+\widehat{K}} K^{\prime} \frac{1}{\sqrt{1+\widehat{K}^{\prime}}}
$$

$\widetilde{\mathrm{Q}}$ is selfadjoint and positive. $\operatorname{det}(1+\mathrm{Q})$ does not vanish, since $\operatorname{det}(1+\mathrm{K})$ does not either (see (53) (55)).

Going back to (90) (after the change (152)), we can show now (for N sufficiently large) :

\section{Proposition 2:}

(i)।

$$
\begin{gathered}
\mathrm{G}_{1 \gamma}|\leq \mathrm{r}(\sigma)| \mathrm{e}^{-\frac{1}{2}<\sigma_{1}, \mathrm{C}_{5} \sigma>\mid} \\
\left|\mathrm{G}_{2 \gamma}\right| \leq \exp \left(-\frac{1}{16}\left(|\ell|+\frac{1}{4}\left|\ell_{1} \backslash \ell\right| \mathrm{N}^{1 / 5}\right) \leq \exp \left(-\mathrm{b}|\Gamma| \mathrm{N}^{1 / 5}\right)\right.
\end{gathered}
$$

with $b=\frac{1}{16} \frac{1}{4(\mathrm{M}+1)^{2}}$ and also

$$
\left|G_{2 \gamma}\right| \leq \exp \left(-\frac{1}{16}|\ell| V\left|N^{1 / 5}-\frac{3}{100}\right| V \mid N-\frac{1}{40} \int_{V} \sigma^{2}(x) d^{2} x\right)
$$

(iii)

$$
\left|G_{3 \gamma}\right| \leq 1
$$


(iv)

$$
\begin{array}{cc}
\text { (iv) } & \left|\mathrm{G}_{4 \gamma}\right| \leq 1 \\
\text { (v) } & \left|\mathrm{G}_{5 \gamma}\right| \leq \exp \mathrm{O}(1)\left\{\frac{1}{\mathrm{~N}}\left(\left|\mathrm{~L}^{1 / 2}\left\|\zeta_{\mathrm{L}}\right\|_{2}+\right| \mathrm{S} \mid+\|\zeta\|_{2}^{2}+\|\zeta\|_{2}\right)+\mathrm{N}^{-1 / 2}\left|\mathrm{~s}_{2}\right|\right\} \\
\text { (vi) } & \left|\mathrm{G}_{6 \gamma}\right| \leq \exp \left\{\mathrm{N}^{-1 / 10}|\mathrm{~S}|\right\} \\
\text { (vii) } & \left|\zeta_{\gamma}\right| \leq 2|\gamma| \mathrm{N}^{1 / 10}
\end{array}
$$

Remark : It is important to note that all these bounds also hold, if we restrict the support of the appearing operators to some subset of squares $\mathrm{Y} \subset \Lambda$ (of course then $\ell, \gamma \cap \Lambda$ etc... have to be restricted to $\mathrm{Y}$ too). And they still hold when we will have introduced the cluster expansion parameters $h$ in the next section, which leave invariant :

1. kernels restricted to a single square or block (see (165), (168))

2. fall-off properties of all kernels (Lemma 5)

3. Positivity properties of all kernels (see (166))

1.3.imply the previous assertion.

Proof:

(i) is trivial, (ii) follows from Lemmas 11,14 and from (35) - (43) and simple geometric considerations which also show that $\mathrm{b}$ can be chosen much larger, apart from the case, where $\ell$ consists of widely isolated $\ell$ fs.

(iii) follows from Lemma 16.

(iv) follows from Lemma 6.

(v) From the bounds in Lemmas 5,4, and Lemma 6 as well as Lemma 13 we find for $C_{3}$

$$
\begin{gathered}
\left|<\zeta_{1}, C_{3} \zeta>\right| \leq O(1) \frac{1}{m} N^{1 / 10} \int_{\left(\text {dist }(x, y) \geq \frac{1}{m} \log N\right)}\left|\zeta_{L}(x)\right| e^{-m \sqrt{2}|x-y|} d^{2} x d^{2} y \\
\left.+\left(\int\left|\zeta_{L}\right| d^{2} x\right) N^{-\sqrt{2}} \frac{1}{m} \log N\right) \leq O(1) \frac{1}{m^{5}} N^{-1.3}\left\|\zeta_{L}\right\|_{1} \leq \\
\leq O\left(\frac{1}{m^{5}}\right) N^{-1.3}|L|^{1 / 2}\left\|\zeta_{L}\right\|_{2} \leq \frac{|L|^{1 / 2}}{N}\left\|\zeta_{L}\right\|_{2}
\end{gathered}
$$

$\mathrm{C}_{4}$ is positive. Similarly we find for $\mathrm{C}_{2}$

$$
\left|<\zeta, C_{2} \zeta>\right| \leq O(1) \frac{1}{m^{2}} \mathrm{~N}_{10}^{\frac{2}{10}} \mathrm{~N}^{-\sqrt{2}} \frac{1}{\mathrm{~m}^{4}}|\mathrm{~S}| \leq \mathrm{N}^{-1}|\mathrm{~S}|
$$

This implies (v) on using $C_{7} \geq 0$, $\left\|\hat{\varepsilon}_{\gamma}\right\| \leq O(1) \frac{1}{N}$ and (70) - (73). 
(vi) Using again the Localization Lemma 13 and Lemma 4 we obtain

$$
\begin{gathered}
\left|\operatorname{Tr}\left(\mathrm{F}_{+}^{2} \mathrm{~F}_{+}^{*}+\mathrm{F}_{+} \mathrm{F}_{+}^{* 2}\right)\right| \leq \mathrm{O}\left(\frac{1}{\mathrm{~m}^{3}}\right) \mathrm{N}^{1-6 / 5}\left|\mathrm{~S}_{+}\right| \\
\left|\operatorname{Tr}\left(\mathrm{F}_{+} \mathrm{F}_{+}^{*}\right)^{2}\right| \leq \mathrm{O}\left(\frac{1}{\mathrm{~m}^{4}}\right) \mathrm{N}^{1-8 / 5}\left|\mathrm{~S}_{+}\right|
\end{gathered}
$$

and similarly for the expansion terms in $\operatorname{det}_{3}\left(1+\mathrm{K}_{ \pm}\right)$

$$
\sum_{n \geq 3}\left|\frac{1}{n} \operatorname{Tr} K_{+}^{n}\right| \leq O(1) \sum_{n \geq 3} \frac{1}{n}\left(\frac{\lambda}{m}\right)^{n} N^{1-\frac{2 n}{5}}\left|S_{+}\right|
$$

which implies (vi)

(vii) is Lemma 3.

QED

The following corrollaries with be useful in the next section.

Corollary 1 : Restricting the volume $\Lambda$ in the functional integral to consist of a single small field square $\Delta$ in $s_{+}$(or in $s_{-}$) we find

$$
\int \mathrm{d} \mu_{\mathrm{o}}^{\Delta}(\zeta) \mathrm{G}_{\mathrm{o}}(\zeta)=1+\mathrm{o}\left(\mathrm{N}^{-\frac{1}{10}}\right)
$$

$\mathrm{d} \mu_{\mathrm{o}}^{\Delta}$ is the normalized Gaussian measure with covariance $\mathrm{C}_{\mathrm{o}}$ restricted to $\Delta$.

Proof: Statements (i), (iii) - (iv) and in particular (vi) of Proposition 2 serve to bound $\mathrm{G}_{\mathrm{o}}(\zeta)$ in $\Delta$. $\mathrm{G}_{30} \equiv \mathrm{G}_{40} \equiv 1$ in our case. The integration in (160) is restricted to $\zeta$ 's fulfilling the small field condition. The remaining $\zeta$ 's for which (in case of $s_{+}$)

$$
\left\|g \zeta_{\Delta} \frac{1}{p^{2}+m^{2}}\left(g \zeta_{\Delta}+m_{\Delta}\right)\right\|_{2} \geq \frac{3}{4} N_{10}^{1}
$$

* an additional factor of $\mathrm{m}$ may be gained on writing

$$
\operatorname{Tr}\left(\mathrm{F}_{+}^{2} \mathrm{~F}_{+}^{*}+\mathrm{F}_{+}^{2} \mathrm{~F}_{+}\right)=\operatorname{Tr}\left(\mathrm{g} \zeta_{+} \frac{1}{\mathrm{p}^{2}+\mathrm{m}^{2}} \mathrm{~g} \zeta_{+}\left(\mathrm{g} \zeta_{+}+\mathrm{m}\right) \frac{1}{\mathrm{p}^{2}+\mathrm{m}^{2}} \mathrm{~g} \zeta_{+}-\left(\mathrm{g} \zeta_{+} \frac{1}{\mathrm{p}^{2}+\mathrm{m}^{2}} \mathrm{~g} \zeta_{+}\right)^{2}\right)
$$

and using (145), the sf condition and Lemma 13 to estimate

$$
\operatorname{Tr}\left(\left(g \zeta_{+} \frac{1}{\mathrm{p}^{2}+\mathrm{m}^{2}} \mathrm{~g} \zeta_{+}\right)\left(\mathrm{g} \zeta_{+}+\mathrm{m}\right) \frac{1}{\mathrm{p}^{2}+\mathrm{m}^{2}} \mathrm{~g} \zeta_{+}\right) \leq \mathrm{O}(1) \mathrm{NO}\left(\frac{1}{\mathrm{~m}^{2}}\right) \mathrm{N}^{-4 / 5} \mathrm{~N}^{-2 / 5}\left|\mathrm{~S}_{+}\right|
$$


fulfill

$$
<\zeta_{\Delta}, \mathrm{C}_{0}^{-1} \zeta_{\Delta}>\geq \mathrm{O}(1) \mathrm{m}^{2} \mathrm{~N}^{1 / 5} \geq \mathrm{N}^{1 / 10+\varepsilon}
$$

which implies the assertion.

Corrollary 2 : For a given large field block $\Gamma_{\mathrm{a}}$ we have

$$
\left|\mathrm{G}_{\gamma}\left(\zeta_{\Gamma_{\mathrm{a}}}\right)\right| \leq \mathrm{e}^{-\mathrm{b} / 2\left|\Gamma_{\mathrm{a}}\right| \mathrm{N}^{1 / 5}}
$$

Proof : the statement follows from (i) - (vii) : Using (ii), (vi) and assuming

$$
\ln 2 \frac{\left|\gamma_{a}\right|}{\left|\Gamma_{a}\right|} N^{1 / 10}-b N^{1 / 5} \leq-\frac{b}{2} N^{1 / 5}-\delta N^{1 / 10}
$$

we have to show in $\ell_{\mathrm{a}} / \mathrm{V}_{\mathrm{a}}$ :

$$
\left|G_{5 \gamma_{a}}\right| \leq \mathrm{e}^{\delta \mathrm{N}^{1 / 10\left|\Gamma_{\mathrm{a}}\right|}}
$$

This follows from (v) on using (129), (144) to estimate $\|\zeta\|$ in $\ell_{\mathrm{a}} / \mathrm{V}_{\mathrm{a}}$ or $\mathrm{S}$. In $\mathrm{V}_{\mathrm{a}}$ we are safe due to (136) anyway (note $\sigma \equiv \zeta$ in $\mathrm{V}_{\mathrm{a}}$ ).

QED

Using the bounds of this chapter we can show in the next that the cluster expansion of our model converges for large $\mathrm{N}$. The restrictions on $\mathrm{N}$ come essentially from the structure of the previous bounds : $\mathrm{N}$ has to be such that the small field quantities (e.g. (158), (159)) give a small factor per volume unit $\Delta$ which has to be small enough to beat all combinatoric factors of the cluster expansion. As was mentioned in the beginning of the paper, $\mathrm{N}$ has to be chosen sufficiently large for given m. From (159) we have e.g. to demand

$$
\mathrm{O}\left(\frac{1}{\mathrm{~m}^{3}}\right) \mathrm{N}^{-1 / 5} \ll 1
$$

which is true if

$$
\frac{1}{\mathrm{~m}^{3}} \mathrm{~N}^{-0.1} \ll 1
$$


Going through the previous Lemmas we see that (163) is the most restrictive condition on $N$ and will be assumed true henceforth (see also (207)).

It goes whithout saying that we did not optimize our expansion techniques with respect to the conditions on $\mathrm{N}$ for the sake of (relative) simplicity.

\section{THE EXPANSIONS, PROOF OF MASS GENERATION}

We now come to the description of the cluster expansion which allows to control the spatial correlations in the model and combined with a subsequent Mayer expansion permits to take the thermodynamic limit and also to bound the decay of the two-point function. Both expansions are only described in so far as the present model requires specific modifications. Otherwise we refer to the literature, $[4,5,9,10]$ and references given there.

\section{IV.1. The cluster expansion, presentation in terms of polymers.}

We use the inductively defined Brydges-Battle-Federbush scheme and refer to [9], ch. III. 1 for a general descrption, many details and notation.

We start from a given large field region $\ell$ and $\Gamma(\ell), \Gamma=\cup_{\mathrm{a}} \Gamma_{\mathrm{a}}$. Given our original partition of the volumes into squares $\Delta_{1}, \Delta_{2}, \ldots$ we divide it into new unions of squares $D_{1}, \ldots, D_{n}, \ldots$ ( in some arbitrary ordering), where for any $i$ either

$$
\begin{gathered}
D_{i}=\Gamma_{a} \text { for some a } \\
\text { or } D_{i}=\Delta_{j} \in \Gamma^{\prime} \text { for some } j \\
\text { or } D_{i}=\hat{\Lambda} .\left(\text { Of course all } D_{i}\right. \text { are different). }
\end{gathered}
$$

That means the whole of a connectivity component $\Gamma_{\mathrm{a}}$ and $\Lambda$ are viewed as single 'blocks', and we will call the $D_{i}$ 'blocks', in the following. We now start from a first $D_{1}$, shortly $D$ and test its coupling with all other blocks in $\widehat{D}=\mathbb{R} / \mathrm{D}$ by introducing a first coupling parameter $h_{1}, 0 \leq h_{1} \leq 1$. We write

$$
\begin{aligned}
P\left(h_{1}\right) & ={ }_{D} P_{D}+\widehat{D} P_{D}+h_{1}\left({ }_{D} P_{D}+\widehat{D} P_{D}\right) \\
& =h_{1} P+\left(1-h_{1}\right)\left({ }_{D} P_{D}+\widehat{D} P \widehat{D}\right),
\end{aligned}
$$

where $P$ or $P(x, y)$ stands for any of the nonlocal kernels from Lemma 5 (items 1,3) to which we apply the expansion. ${ }_{D}{ }^{P}{ }_{D}$ stands for $\chi_{D}(x) P(x, y) \chi_{D}(y)$ etc. Thus $P(1)=P$ and $P(0)$ completely 
decouples $\mathrm{D}$ from $\widehat{\mathrm{D}}$. One of the merits of (165) lies in the fact that

$$
\mathrm{a} \leq \mathrm{P}\left(\mathrm{h}_{1}\right) \leq \mathrm{A} \text {, if } \mathrm{a} \leq \mathrm{P}(1) \leq \mathrm{A} \text { (in the operator sense) }
$$

that is to say, the interpolation procedure respects the positivity properties. The analogue of (166) also holds if we introduce more and more coupling parameters $h_{2}, \ldots, h_{n}$, (see below)). (cf. the remark after Proposition 2).

The functional integrals $\mathrm{I}_{\gamma}$ in (90) may then be written as

$$
I_{\gamma}=I_{\gamma}\left(h_{1}=1\right)=I_{\gamma}(0)+\int_{0}^{1} d h_{1} \frac{\partial}{\partial h_{1}} I_{\gamma}\left(h_{1}\right)
$$

In the first term $\mathrm{D}$ is decoupled from $\widehat{\mathrm{D}}$. In the second the derivative (acting on terms as (165)) creates an explicit link between $\mathrm{D}$ and $\widehat{\mathrm{D}}$. For the first term we then start again as before, choosing a new block in $\widehat{\mathrm{D}}$ and a new coupling parameter $\mathrm{h}$. The second term may be written as a sum over the blocks in $\widehat{D}$ linked to $\mathrm{D}$ via the derivative link. Picking a particular block $\mathrm{D}^{(2)}$ in this sum we introduce a new coupling parameter $h_{2}$ and test the coupling of $D \cup D^{(2)}=D^{12}$ and its complement $\widehat{D}^{12}$ via $h_{2}$. Writing $\mathrm{P}^{(1)}$ for $\mathrm{P}\left(\mathrm{h}_{1}\right)$ we set, following (165)

$$
\mathrm{P}^{(1)}\left(\mathrm{h}_{2}\right)=\mathrm{h}_{2} \mathrm{P}^{(1)}+\left(1-\mathrm{h}_{2}\right)\left(_{12} \mathrm{P}_{12}^{(1)}+\underset{(12)}{\mathrm{P}^{(1)}} \widehat{(12)}\right)
$$

We may then again write a Taylor formula as (167) and go on as before until the blocks are exhausted. Once a term with $h_{i}=0$ is chosen the blocks treated so far are decoupled from the rest. The union of these blocks, say $n+1$, is called a polymer $Y$, the factorized contribution is its activity $\mathrm{A}(\mathrm{Y})$. We thus have

$$
\mathrm{A}(\mathrm{Y})=\sum_{\mathrm{T}} \int \mathrm{dh} \mathrm{M}_{\mathrm{T}^{\prime}}(\mathrm{h}) \partial_{\mathrm{h}}^{\mathrm{n}} \int \mathrm{d} \mu_{\gamma}^{\mathrm{Y}}(\mathrm{h}) \mathrm{G}_{\gamma}^{\mathrm{Y}}(\mathrm{h})
$$

$$
A_{e}(Y)=\sum_{T^{\prime}} \int d h M_{T^{\prime}}(h) \partial_{h}^{n} \int d \mu_{\gamma}^{Y}(h) F_{2}(h) G_{\gamma}^{Y}(h)
$$

with the following explanations :

1. All h parameters are collectively denoted by $h$.

2. $A_{e}(Y)$ denotes an 'external polymer' : $Y$ contains the support of the test functions $f_{1}, f_{2}$. One may convince oneself (see below) that both have to belong to the same polymer (fermion number 
conservation).

3. All kernels and fields are now restricted to $\mathrm{Y}$. In particular $\mathrm{d} \mu_{\gamma}^{\mathrm{Y}}$ denotes the (normalized) Gaussian measure with covariance

$$
\begin{gathered}
C_{\gamma}^{Y}(h)=C_{o}(h)+C^{(\gamma)}(h), \\
C^{(\gamma)}(h)=S(h) \widehat{C}_{\gamma} S(h)(\operatorname{see}(101),(102)),
\end{gathered}
$$

where $S(h)$ is the kernel of $\frac{1}{\sqrt{\mu^{2}+\pi_{\text {ren }}}}$ together with the h-parameters (see $\left.(165),(168), \ldots\right)$ and restricted to $Y$.

4. As is seen in (170) the h's are introduced twice in $C^{(\gamma)}$. This is necessary to achieve factorization. If we introduced them only for $\mathrm{C}^{(\gamma)}$ as a whole, the two kernels $\mathrm{S}$ could link two points in $\mathrm{Y}$ to any point in $\widehat{\mathrm{Y}}$. Note that we don't have to expand $\frac{1}{1+\mathrm{f}}$ due to our definition of the blocks, the definition of $\Gamma_{a}, \gamma_{a}(44)$ and the compact support of $\frac{1}{1+f}(63)$ which was just introduced for this purpose (otherwise a more complicated procedure would be necessary [11] ). We also expand each square root in (87). This then guarantees that $\mathrm{A}(\mathrm{Y})$ for given $\mathrm{Y}$ is completely factorized from the other polymers, and its covariance is independent of the large or small field assignments in all other polymers. Due to our expansion rules and the 'security belts' around the large field squares we need not introduce the h's twice in $\mathrm{C}_{0}$ (using (63)).

5. (169) contains an integral over h's together with a sum over ordered (rooted) trees $\mathrm{T}^{\prime}$ accompanied by a combinatoric factor $\mathrm{M}\left(\mathrm{T}^{\prime}\right)$ [9]. $\mathrm{T}^{\prime}$ indicates in which way the blocks have been successively connected. We have

$$
\sum_{T, T\left(T^{\prime \prime}\right)=T} \int M\left(T^{\prime}\right)(h) d h=1
$$

where $\mathrm{T}$ now is the unordered (rooted) tree belonging to $\mathrm{T}$. Therefore we do not comment on $\int \mathrm{dh}, \sum_{\mathrm{T}^{\prime}(\mathrm{T})}, \mathrm{M}\left(\mathrm{T}^{\prime}\right)$ any further and refer to the liberature $[9,10,12]$.

The derivatives in (169) may apply to $d \mu_{\gamma}(h)$ or to $F_{2}(h) G_{\gamma}(h)$. Application with respect to $d \mu_{\gamma}(h)$ is evaluated by partial integration ([5], ch.9):

$$
\partial_{\mathrm{h}_{\mathrm{i}}} \int \mathrm{d} \mu_{\gamma}^{\mathrm{Y}}(\mathrm{h}, \zeta) \ldots=\int \mathrm{d} \mu_{\gamma}^{\mathrm{Y}}(\mathrm{h}, \zeta)<\frac{\delta}{\delta \zeta}, \partial_{\mathrm{h}_{\mathrm{i}}} \mathrm{C}_{\gamma}(\mathrm{h}) \frac{\delta}{\delta \zeta}>\ldots
$$


where the $\zeta$-derivatives now apply to the rest... The supports of the derived kernels, here $\partial_{h_{i}} S(h)$, are by construction restricted to the two blocks which are linked by $h_{i}$ according to the given tree $T$. This is crucial for bounding $\mathrm{A}(\mathrm{Y})$. For (172) it means that the $\zeta$-derivatives are localized in these blocks, more precisely they are either

(i) strictly localized

(ii) essentially localized.

The latter denotes the case where e.g. $\partial_{h_{i}}$, applies to the first $S(h)$ in $C^{(\gamma)}$, and we regard the $\frac{\delta}{\delta \zeta}$ standing on the right. It is linked to its localization block via the underived, but exponentially decaying second $S(h)$.

For simplicity we will treat in the following both $\zeta$-derivatives as if localized in a single block : the exponential decay allows to bound the effect of essential localization by an additional $\mathrm{O}\left(\frac{1}{\mathrm{~m}^{2}}\right)$ per such derivative.

The derivatives $\partial_{h_{i}}$ also apply to $G_{i \gamma}$ in particular to the kernels in the Fredholm determinants. Their outcome will be treated in the next subsection. Our presentation of the model has been judiciously chosen such that all derivatives will generate a small factor, i.e. a negative power of $\mathrm{N}$, per small field square so that the expansion converges (in the large field regions we have the suppression factors (161)).

Application of the cluster expansion to (90) thus puts it into the following form

$$
\mathrm{S}_{2}\left(\mathrm{f}_{1}, \mathrm{f}_{2}\right)=\frac{\sum_{\ell, \mathrm{s}_{+}} \prod_{\mathrm{a}} \mathrm{Z}_{\gamma_{\mathrm{a}}} \sum_{\mathrm{q},\left[\mathrm{Y}_{\mathrm{i}}^{\ell}\right)} \frac{1}{\mathrm{q} !} \prod_{\mathrm{i}=1}^{\mathrm{q}} \mathrm{A}\left(\mathrm{Y}_{\mathrm{i}}^{\ell}, \mathrm{f}_{1}, \mathrm{f}_{2}\right)}{\sum_{\ell, \mathrm{s}_{+}} \prod_{\mathrm{a}} \mathrm{Z}_{\gamma_{\mathrm{a}}} \sum_{\mathrm{q},\left\{\mathrm{Y}_{\mathrm{i}}^{\ell}\right)} \frac{1}{\mathrm{q} !} \prod_{\mathrm{i}=1}^{\mathrm{q}} \mathrm{A}\left(\mathrm{Y}_{\mathrm{i}}^{\ell}\right)}
$$

We again join a series of explanations :

1. The polymers $Y_{i}^{\ell}, i=1, \ldots, q$ are unions of $D_{j}^{j} s(164)$ and therefore depend on $\ell, s_{+}$(we only indicate the first). They obey $\bigcup_{i} Y_{i}^{\ell}=\mathbb{R}^{2}, Y_{i}^{\ell} \cap Y_{j}^{\ell}=0$ for $i \neq j$. The $D_{j}$ in $Y_{i}^{\ell}$ are linked via derived propagators according to a given tree $\mathrm{T}$ (see above).

2. In the numerator there is one external polymer $A_{e}\left(Y_{i}^{\ell}, f_{1}, f_{2}\right)$. The others do not depend on $f_{1}$, $\mathrm{f}_{2}$ (with apologies for the shorthand notation). 
3. According to (103) $Z_{\gamma}=\prod_{a} Z_{\gamma a}$, which we used above. Since $\left(\gamma_{a} \cap \Lambda\right) \subset \Gamma_{a}(40,43)-$ so that each $\gamma_{a} \cap \Lambda$ is contained in exactly one $D_{j}$ - we may rewrite (174) as

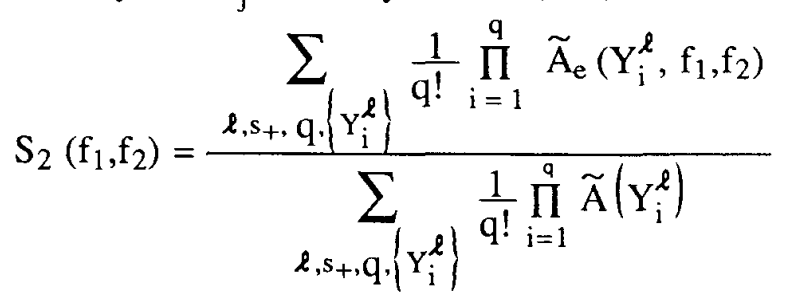

where the $Z_{\gamma_{a}}$ have been absorbed in the $A\left(Y_{i}^{\ell}\right)$ to which they belong, i.e. for which $\left(\gamma_{a} \cap \Lambda\right) \subset Y_{i}^{\ell}$. This means

$$
\widetilde{A}(Y):=A(Y) \prod_{a} Z_{\gamma a},
$$

where the product is over over all a, for which $\gamma_{a} \cap \Lambda \subset \mathrm{Y}$.

4. We shall not write a completely explicit expression for the amplitudes of the polymers A (Y), which would be very lengthy. For a relatively explicit representation in the simpler case of a $\varphi_{4}^{4}$ theory see [9], ch. III. 1.

The simplest example for $\mathrm{A}\left(\mathrm{Y}_{\mathrm{i}}^{\ell}\right)$ is that one where $\mathrm{Y}_{\mathrm{i}}^{\ell}$ is a single small field square $\Delta$ in $\mathrm{s}_{+}$or in $\mathrm{s}_{-}$. Due to $(160)$ we find in this case

$$
A(\Delta)=1+o\left(N^{-1 / 10}\right)
$$

It is convenient therefore to introduce for a polymer $\mathrm{Y}$

$$
\mathrm{a}(\mathrm{Y})=\frac{\widetilde{\mathrm{A}}(\mathrm{Y})}{\prod_{\Delta \subset \mathrm{Y}} \mathrm{A}(\Delta)}
$$

so that

$$
S_{2}\left(f_{1}, f_{2}\right)=\frac{\sum_{\ell, s_{+}, q,\left\{Y_{i}^{\ell}\right\}} \frac{1}{q !} \prod_{i=1}^{q} a\left(Y_{i}^{\ell}, f_{1}, f_{2}\right)}{\sum_{\left.\ell, s_{+}, q, \mid Y_{i}^{\ell}\right\}} \frac{1}{q !} \prod_{i=1}^{q} a\left(Y_{i}^{\ell}\right)}
$$


where now $Y_{i}^{\ell} \cap Y_{j}^{\ell}=0$ for $i \neq j$ and $\bigcup_{i} Y_{i}^{\ell} \subset \mathbb{R}^{2}$ (instead of equality), since we omit factors of 1 and demand that $Y_{i}^{\ell}$ does not consist of a single $\Delta$ unless it is an external one (i.e. contains the support of $f_{1}$ or $f_{2}$ (in the numerator)).

\section{IV.2. Description of the Mayer expansion}

To prove the existence of $S_{2}\left(f_{1}, f_{2}\right)$ (or of any $n$-point function) we have to factorize the denominator from the numerator in (177) and to perform the division, since the denominator diverges in the thermodynamic limit by translation invariance. This elimination of the vacuum polymers is performed with the help of the Mayer expansion. The Mayer expansion is taylored to free the sum over polymers from disjointness constraints, which then allows factorization. For a general description of the Mayer expansion we refer again to [9], ch. III. 1 and [10]. Here we concentrate on the complications due to the case at hand.

Namely, in the simplest case the disjointness constraint among polymers is just the condition that they have no common block (disjoint support). Here we have not only a sum over Y's as sums of squares, but also sums over $\ell, s_{ \pm}$assignments. First we have to cast these sums into a single one. Once this has been done, we have to analyze the constraints among the polymers. It is not difficult to cope with the complications coming from constraints which have a finite range (instead of the zero range of the simple disjointness condition). Constraints restricting polymers at an arbitrarily distance are disastrous for the convergence proof of the Mayer expansion however. Such constraints can in principle arise here, if we first sum over polymers as sets of squares and then over the possible $\ell, s_{ \pm}-$assignments. Take e.g. an s-square $\Delta$ belonging to some polymer $\mathrm{Y}$. Assume there exists a continuous path joining $\mathrm{x} \in \Delta$ to $\mathrm{y} \in \Delta^{\prime}$, where $\Delta^{\prime}$ is another, arbitrarily distant, square, which has been assigned $s_{+}$. If the path never touches a square from $\ell_{1}(35,36), \Delta$ has to be assigned $s_{+}$too $; s_{-}$is excluded. If instead the path crosses once an (arbitrarily distant) contour of squares from $\ell_{1}$, only s. will be allowed, and so on... These constraints, being of topological nature, have infinite range. We avoid this problem with the help of a modification of the cluster expansion which we only mention in this section though, being fully logical, it should have been introduced earlier. We once more enlarge the $\Gamma_{\mathrm{a}}$ by setting

$$
\widetilde{\Gamma}_{\mathrm{a}}=\Gamma_{\mathrm{a}} \cup \operatorname{int} \ell_{1}\left(\Gamma_{\mathrm{a}}\right)
$$

Here int $\ell_{1}\left(\Gamma_{\mathrm{a}}\right)$ is the set of all squares of $\Lambda$, which are surrounded by closed contours of squares from $\ell_{1} \subset \Gamma_{\mathrm{a}}$; i.e. we add to $\Gamma_{\mathrm{a}}$ the interior of $\ell_{1}$ - contours. If some $\widetilde{\Gamma}_{\mathrm{a}^{\prime}}$ is then contained in $\widetilde{\Gamma}_{\mathrm{a}}$, we cancel $\widetilde{\Gamma}_{\mathrm{a}^{\prime}}$ from the set of the $\widetilde{\Gamma}_{\mathrm{i}}$ (by definition).

Now we assume the blocks D of the cluster expansion are not as in (164), but 


$$
\mathrm{D}_{\mathrm{i}}=\widetilde{\Gamma}_{\mathrm{a}} \text { for some } \mathrm{a} \text {, or } \mathrm{D}_{\mathrm{i}}=\Delta_{\mathrm{j}} \in \widetilde{\Gamma}^{\prime} \text { for some } \mathrm{j}
$$

(of course $\widetilde{\Gamma}_{\mathrm{a}}$ may contain several $\Gamma_{\mathrm{a} 1}, \ldots, \Gamma_{\mathrm{ak}}$ ). With this assumption there are no more topological constraints between distinct polymers ; we transform the sums in (177) as follows :

$$
\sum_{\ell, s_{+} q,\left\{Y_{i}^{\ell}\right\}} \frac{1}{q !} \prod_{i=1}^{q} a\left(Y_{i}^{\ell}\right)=\sum_{q,\left\{Y_{i}\right\}} \frac{1}{q !} \prod_{i=1}^{q} b\left(Y_{i}\right)
$$

with the explanations :

(i) The right sum is over all sets $\left\{Y_{1}, \ldots, Y_{q}\right\}$, where the $Y_{i}$ are sets of $\Delta$ 's, a single $\Delta$ being excluded if it is not an external one, with : $Y_{i} \cap Y_{j}=0$ for $i \neq j, U_{j} Y_{j} \subset \mathbb{R}^{2}$. (One $Y_{j}$ contains $\widehat{\Lambda}$ ).

(ii) $b\left(Y_{i}\right)$ is calculated from a $\left(Y_{i}^{1}\right)$ through

$$
\mathrm{b}\left(\mathrm{Y}_{\mathrm{i}}\right)=\sum_{\left(\ell, \mathrm{s}_{+}\right)\left(\mathrm{Y}_{\mathrm{i}}\right)} \mathrm{a}\left(\mathrm{Y}_{\mathrm{i}}^{\ell}\right)
$$

where the sum is over all assignments $\ell, \mathrm{s}_{+}, \mathrm{s}_{-}$compatible with $\mathrm{Y}_{\mathrm{i}}\left(\mathrm{s}_{-}=\mathrm{Y}_{\mathrm{i}} \backslash\left(\ell \cup \mathrm{s}_{+}\right)\right)$, i.e... Given any assignment $\ell, s_{+}, s_{-}$to the squares of $Y_{i}$, we define $\ell_{1}\left(Y_{i}\right)$ as in (35), (36), but restricting to $Y_{i}$ instead of $\Lambda$. Any assignment for which there exists some

$$
\Delta \in \ell_{1}\left(\mathrm{Y}_{\mathrm{i}}\right) \text { with dist }\left(\Delta,\left(\partial \mathrm{Y}_{\mathrm{i}} \backslash \partial \Lambda\right)\right) \leq \mathrm{M}
$$

is forbidden. For the remaining assignments we form the blocks $\widetilde{\Gamma}_{\mathrm{a}}$, on assuming that the complement of $Y_{i}$ in $\Lambda$ consists exclusively of small field squares, i.e. $\Lambda \backslash Y_{i} \subset \Lambda \backslash \ell_{1}$. If all $\widetilde{\Gamma}_{a}$ fulfill

$$
\tilde{\Gamma}_{\mathrm{a}} \subset \mathrm{Y}_{\mathrm{i}}
$$

the assignment is admitted and called compatible with $\mathrm{Y}_{\mathrm{i}}$, otherwise it is forbidden. It is now straightforward to check that this definition of compatibility is the one for which (181) is satisfied, and it is completely local. It implies that all $\Delta$ with $\Delta \cap\left(\partial Y_{i} \backslash \partial \Lambda\right) \neq 0$ are small field and of equal sign for any connected component of $\partial Y_{i} \mid \partial \Lambda$. Due to our boundary conditions these 
squares are (if they exist) $s_{+}$, (and we do not sum over the opposite assignment $\left(s_{+} \rightarrow s_{-}\right.$) for them).

(181) has now the form required for the application of the Mayer expansion in standard way. On applying it we thus divide by the vacuum functional to obtain (in the notation of [9])

$$
S_{2}\left(f_{1}, f_{2}\right)=\sum_{M=\left(f_{1}, f_{2}\right)-\text { configuration }} b^{T}(M),
$$

where $M$ is any sequence of overlapping polymers $Y_{1}, \ldots, Y_{q}$, and one polymer is external, i.e. contains the supports of $f_{1}, f_{2}$ and the factor $F_{2}(\zeta)$ from $(90) . b^{T}(M)$ is given by

$$
b^{T}(M)=\left(\frac{1}{q !} \prod_{i=1}^{q} b\left(Y_{i}\right)\right) T(M)
$$

and the combinatorial factor $\mathrm{T}(\mathrm{M})$ stems from the organization of the Mayer expansion terms through trees.

The sufficient condition for the convergence of (181) in the thermodynamic limit is a bound on the amplitudes $b(Y)$, when summing over all $\mathrm{Y}$ containing a given square or point to break translation invariance (which is broken in (184) through the external squares).

We state it as

\section{Proposition 3 :}

$$
\left|\sum_{Y, 0 \in Y \subset \Lambda} b(Y) e^{|Y|}\right| \leq 1 / 2
$$

for $\mathrm{N}$ sufficiently large, uniformly in $|\Lambda|$.

The proof is in sect IV.4, using the results of sect. IV.3. For $\mathrm{N}$ large it is also true on replacing e by larger constants. $|\mathrm{Y}|$ is the number of $\Delta$ 's (i.e. the volume) of $\mathrm{Y}$.

In the remainder of this subsection we have to free us, however, again from the enlargement (178),

$$
\Gamma_{\mathrm{a}} \rightarrow \widetilde{\Gamma}_{\mathrm{a}},
$$

now that the Mayer expansion has been performed. The reason for this is that from Proposition 2 (155) we obtain a suppression factor $\exp \left(-\mathrm{b}|\Gamma| \mathrm{N}^{1 / 5}\right)$ in the large field region. But this factor is not sufficient to prove Proposition 3, if we stay with $\widetilde{\Gamma}$ instead of $\Gamma$, since it may happen that $\left.\widetilde{\Gamma}|\sim| \Gamma\right|^{2}$ 
(take e.g. the case where precisely the squares on the boundary of $\Lambda$ are in $\ell$. Then $\tilde{\Gamma}=\Lambda$, whereas $\left.|\Gamma| \sim 4 \mathrm{M}|\Lambda|^{1 / 2}\right)$. Then

$$
\mathrm{e}^{|\mathrm{Y}|} \sim \mathrm{e}^{\tilde{|\Gamma|}} \sim \mathrm{e}^{|\Gamma|^{2}}>\mathrm{e}^{\mathrm{N}^{1 / 5}|\Gamma|}
$$

for large $|\Gamma|$. So we proceed as follows. After performing the Mayer expansion we cut up again

$$
\widetilde{\Gamma}_{\mathrm{a}}=\underset{\mathrm{i}=1}{\mathrm{j}} \Gamma_{\mathrm{a}_{\mathrm{i}}} \cup \underset{\Delta \in \widetilde{\Gamma}_{\mathrm{a}}, \notin \Gamma_{\mathrm{a}_{\mathrm{i}}}}{\mathrm{U}},
$$

and perform a BBF-cluster expansion of $\tilde{\Gamma}$ w.r.t. the $\Gamma_{\mathrm{ai}}$, and the $\Delta$. The result is again organized by new trees linking the $\Gamma_{\mathrm{ai}}$ and $\Delta$ 's from (186). There are also contributions where $\widetilde{\Gamma}_{\mathrm{a}}$ factorizes into several parts. The whole of $\widetilde{\Gamma}_{\mathrm{a}}$ is linked via branches of the original tree to $Y \widetilde{\Gamma}_{\mathrm{a}}$, the number of those being given by the coordination number of the original tree at $\widetilde{\Gamma}_{a}$. For any of these branches we have to sum now over all possibilities to which $\Gamma_{\mathrm{aj}}$ or $\Delta$ this branch now links his block from $\mathrm{Y} \backslash \widetilde{\Gamma}_{\mathrm{a}}$. Any of these choices corresponds to a unique tree for the system of blocks (186) in $\mathrm{Y}$, where however still some sets of blocks may be completely factorized from $\mathrm{Y} \backslash \Gamma_{\mathrm{a}}$ (namely if no branch from $Y \backslash \widetilde{\Gamma}_{\mathrm{a}}$ hits any block of a contribution which was factorized before).

Comparing to the case where we expand $\left\{\Gamma_{\mathrm{a}_{\mathrm{i}}}, \Delta\right\}$ (186) from the beginning, we have two differences : First we have to sum also over the aforementioned factorized contributions. On the other hand certain trees will not appear due to the detour via $\tilde{\Gamma}_{\mathrm{a}}$, namely those where some block from $\mathrm{Y} \backslash \widetilde{\Gamma}_{\mathrm{a}}$ is linked to several elements of (186) (r.h.s.) at the same time : this is because these do not correspond to a tree, when collapsing $\left\{\Gamma_{\mathrm{a} i}, \Delta\right\}$ to $\widetilde{\Gamma}_{\mathrm{a}}$ but contain loops. Since we are proving Proposition 3 by summing over trees $\mathrm{T}$ and polymers $\mathrm{Y}$ and estimating the individual contributions in modulus, leaving out certain trees can only improve the bounds. But how do we deal with the new factorized contributions within $\widetilde{\Gamma}_{\mathrm{a}}$ ?

The easiest way seems to be to factorize them within each given $\widetilde{\Gamma}_{\mathrm{a}}$ - for given $\mathrm{Y} \supset \widetilde{\Gamma}_{\mathrm{a}}$ and given assignment $\ell, \mathrm{s}_{+}-$via a new Mayer expansion. External configurations are now those which contain either an external square $\Delta\left(f_{1}\right), \Delta\left(f_{2}\right)$ or a block linked to $Y \widetilde{\Gamma}_{a}$. Given then an entry in the sum (182) for which the decomposition (186) for $\widetilde{\Gamma}_{\mathrm{a}} \neq \varnothing$ is not trivial we find (see again [9]) 


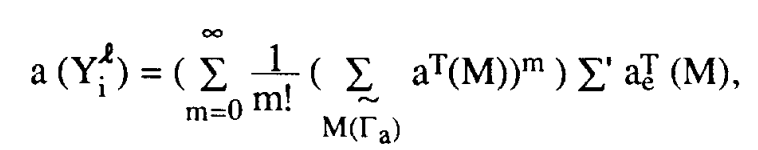

where the second sum is over the external Mayer configurations and therefore is the one which fits back into our description in terms of trees and Y's in the cluster expansion (164), as explained above. The first factor contains the vacuum Mayer configurations within $\tilde{\Gamma}_{\mathrm{a}}$.

Using again [9], ch. III. 1 we estimate

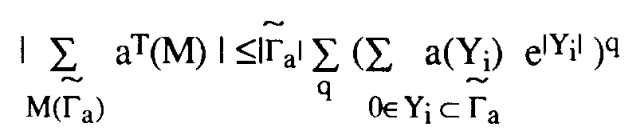

$\left(\widetilde{\Gamma}_{\mathrm{a}} \mid\right.$ takes care of translation invariance within $\tilde{\Gamma}_{\mathrm{a}}$, which is then broken in the sum over $\mathrm{Y}_{\mathrm{i}}$ by demanding that it contains a given point ( $=0$ without restriction); note that the assignment of $Y_{i}$ is fixed).

Following step by step the proof of Proposition 3, the expression in parentheses is $\leq 1 / 2$. But we have a much better estimate from the fact that always $\varnothing \neq \Gamma_{\mathrm{a}} \subset Y_{\mathrm{i}}$. We may keep aside a factor

$$
\mathrm{e}^{-\mathrm{b} / 4}\left|\Gamma_{\mathbf{a}}\right| \mathrm{N}^{1 / 5}
$$

and proceed as in the proof of Proposition 3, using only the rest of the large field suppression to verify the bound. Then

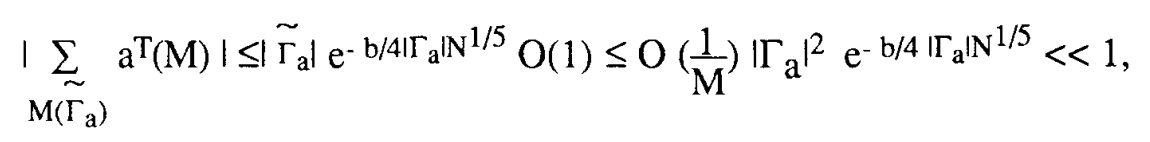

and the exponential of (189) is thus $\approx 1$.

So in the following we will forget about the $\widetilde{\Gamma}_{\mathrm{a}}$ and give the proof of Proposition 3 for the polymers of the original expansion (164).

Before proving Proposition 3, we have to look at the expression for $\mathrm{a}\left(\mathrm{Y}^{\ell}\right)$ in more detail. This means that we have to look at the outcome of the cluster expansion derivatives in the functional integral.

\section{IV.3 The outcome of the derivatives}

We apply the $h$ - or $\zeta$ - derivatives to the various $G_{\gamma}$ from (90). We first shortly comment on $G_{1 \gamma}$ 
$\mathrm{G}_{4 \gamma}, \mathrm{G}_{5 \gamma}$, which are relatively simple to handle and of a technical nature. The remaining terms are treated more extensively.

G1 $\gamma$ : We start with the boundary terms which factorize over the squares $\Delta$ and are therefore independent of the h-parameters. Applying $\zeta$-derivatives we find

(i) for b( $\sigma)$ a factor $\theta^{\prime}\left(\int_{\Delta} \sigma\right)$ which is bounded by $O(1)$ if $\zeta$ is an $\ell$-square and vanishes for ssquares due to Lemma 14.

(ii) Deriving

$$
\frac{\delta}{\delta \zeta(\mathrm{x})} \theta\left(\frac{\left\|\mathrm{A}_{\Delta}\right\|_{2}}{\mathrm{~N}^{\alpha}}-1\right)=\theta^{\prime}\left(\frac{\left\|\mathrm{A}_{\Delta}\right\|_{2}}{\mathrm{~N}^{\alpha}}-1\right) \mathrm{N}^{-\alpha} \frac{\delta}{\delta \zeta}\left\|\mathrm{A}_{\Delta}\right\|_{2} \leq \mathrm{O}(1) \mathrm{N}^{-\frac{1}{10}} \quad\left(\alpha=\frac{1}{10}\right)
$$

(The first factor restricts $\Delta$ to have $3 / 4 \mathrm{~N}^{\alpha} \leq\left\|\mathrm{A}_{\Delta}\right\|_{2} \leq 5 / 4 \mathrm{~N}^{\alpha}$. So by Lemma 13 the last factor is easily bounded by $\mathrm{O}(1)$ if the first does not vanish).

Now we come to $r(\sigma) e^{\left.-\frac{1}{2}<\sigma, C_{5} \sigma\right)}$. These terms may be replaced by 1 unless the polymer $\mathrm{Y}$ contains $\hat{\Lambda}$. In the latter case the $h$ - or $\zeta$ - derivative(s) associated to $\hat{\Lambda}$ will always produce at least one $\zeta$ - field "localized" in $\hat{\Lambda}$. At this stage we may then take the limit $\mathrm{R} \rightarrow \infty$ (26) to see that such a polymer gives a vanishing contribution, and we therefore assume from now on

$$
\mathrm{Y} \subset \Lambda \text {. }
$$

$\mathrm{C}_{5}$ restricted to $\mathrm{Y}$ then vanishes as does the restriction of $(\sigma \hat{\Lambda})^{2}$, and we may from now on forget about these terms.

$\mathbf{G}_{\mathbf{4} \gamma}, \mathbf{G}_{\mathbf{5} \gamma}(95,96,152)$ : Both terms are at most quadratic in $\zeta$. Derivatives of the quadratic terms with respect to $h$ or $\zeta$ generate at most two $\zeta$-fields and a distance factor. This factor is bounded by $\mathrm{N}^{-1}$ for $\mathrm{G}_{5 \gamma}$ due to Lemma 6 . Due to our definition of the $\mathrm{D}_{\mathrm{j}}$ we also get $\mathrm{N}^{-1}$ for $\mathrm{G}_{4 \gamma}$ if the derivative is associated to a small field block $\Delta$ since (see (44)) dist $(\Delta, \gamma) \geq \frac{M}{2}$. Only if a $\zeta$-derivative is associated to some $\Gamma_{\mathrm{a}}$ this distance factor may be $O(1)$. The $\zeta$-fields generated by the derivatives are linked to the blocks of the corresponding derivatives by exponentially decreasing kernels, so again are (essentially) localized in these blocks. If $\delta_{\zeta(\mathrm{x})}$ hits the linear term in $\mathrm{G}_{5}$ it generates $\sigma_{\mathrm{o}} \mathrm{h}_{\Delta}(\mathrm{x})$, which on integrating over the respective block is bounded by $\sim \mathrm{N}^{-1} \mid \mathrm{DI}$ (see (60), (70), (71)). Successive $\zeta$-derivatives may also hit $\zeta$-fields already descended by derivation. This leads to a factorially increasing number of terms, even more so when treating the subsequent $\mathrm{G}_{\gamma}{ }_{\gamma} \mathrm{s}$ (see below). This problem is already present in $\mathrm{P}(\varphi)$ - theories. It is solved by the so-called local factorial principle (LFP) : the descended $\zeta$ 's are essentially localized in their blocks. These blocks 
have to be different from each other, apart from the case where the derivatives are associated to links in the respective tree which all join the same block. So the maximal number of derivatives localized in the same block is given by the coordination number $d$ of the tree at that block. This implies that the distances to be covered by the subsequent links from this block to their new endpoints have to grow more and more for large $d$, in two dimensions up to $O(\sqrt{d})$, so that the distance factor of order

$$
\prod_{i=1}^{\mathrm{O}(\sqrt{d})} \mathrm{e}^{-\eta m i} \sim \mathrm{e}^{-\mathrm{O}(1) \eta m \mathrm{~m}^{3 / 2}}
$$

becomes smaller than any power of (d!), even if we use only a fraction $\eta$ of the decay of the kernels. If $d$ is not large many $\zeta$ 's may still accumulate in one block different from their localization blocks, namely if they are only essentially localized there. Then a factor as (190) arises from the exponential decrease of $S(h)(170)$ between these blocks and the former one. This latter case, technically not much different from the first, appears only due to our twofold expansion of the covariance $\mathrm{C}^{(\gamma)}$ in case of nonempty large field contributions.

If a $\zeta$-derivative hits some already descended $\zeta$-field from $\mathrm{G}_{4}$ or $\mathrm{G}_{5}$, the new derivative does not generate a new small factor. So the net small factor per small field derivative is not $\sim \mathrm{N}^{-1}$ but $\sim \mathrm{N}^{-1 / 3}$ (one $\mathrm{h}$ and two subsequent $\zeta$-derivatives in the worst case). This is similar as in $g \varphi^{4}$ where the gain per derivative is not $\mathrm{g}$ but $\mathrm{g}^{1 / 4}$.

Among the remaining terms we now look at

$$
\exp \left\{-\frac{1}{2} \operatorname{Tr}\left(\mathrm{F}_{+}^{2} \mathrm{~F}_{+}^{*}+\mathrm{F}_{+} \mathrm{F}_{+}^{* 2}+\frac{1}{2}\left(\mathrm{~F}_{+} \mathrm{F}_{+}^{*}\right)^{2}+(+\leftrightarrow-)\right\}(\operatorname{see}(52))\right.
$$

A $\zeta$ - or $h$ - derivative will bring down a factor $\leq \mathrm{O}\left(\frac{1}{\mathrm{~m}^{3}}\right) \mathrm{N}^{-1 / 5}=\mathrm{o}\left(\mathrm{N}^{-1 / 10}\right)$ (see (158)). All descended $\zeta$-fields $\left(\zeta \equiv \tau\right.$ in $\left.S_{ \pm} !\right)$are joined via exponentially decreasing kernels to the respective block of the derivative. So the LFP works as before. New derivatives hitting descended $\zeta$ 's generate an additional $\mathrm{N}^{-1 / 10}$ on replacing (in the estimates) $\int_{\Delta} g|\zeta| \leq \mathrm{O}\left(\frac{1}{\mathrm{~m}}\right) \mathrm{N}^{-2 / 5}$ by $\mathrm{g} \sim \mathrm{N}^{-1 / 2}$. The term $\left(\mathrm{F}_{+} \mathrm{F}_{+}^{*}\right)^{2}$ is suppressed by an additional $\mathrm{N}^{-2 / 5}$ (see (158)).

Now it remains to discuss the determinants $\operatorname{det}_{3}^{1 / 2}\left(1+\mathrm{K}_{ \pm}\right), \operatorname{det}^{1 / 2}(1+\mathrm{Q}), \operatorname{det}_{2}^{1 / 2}\left(1+\mathrm{A}_{\mathrm{La}}\right)($ see $(50)$ (55), (94), (97), (152), (155)). For the two $\operatorname{det}_{3}$-terms we have in principle two choices for the treatment : since the $\zeta$-fields are in the small field region we may expand in powers of $\operatorname{Tr} \mathrm{K}_{ \pm}^{\mathrm{n}}, \mathrm{n} \geq 3$ and then perform the derivatives. The outcome can be estimated using (159), Lemma 13 and the LFP. This does not work for the other terms since $\mathrm{K}^{\prime}, \mathrm{A}_{\mathrm{La}}$ are not bounded. So we use a different 
method. We will first deal with $\operatorname{det}^{1 / 2}(1+Q)$, since this is the most complicated term.

$\operatorname{det}^{1 / 2}(1+Q):$

Using Lemma 16 we write $\operatorname{det}^{1 / 2}(1+Q)=\operatorname{det}^{1 / 4}(1+Q) \operatorname{det}^{1 / 4}\left(1+Q^{*}\right)$ and apply the BBF expansion to the r.h.s. Note that the organization of the expansion terms in trees requires that the derivatives are always applied to symmetric expressions (here the product of the two determinants). Once this is assured there is however no difference in the discussion of $\mathrm{Q}$ and $\mathrm{Q}^{*}$, so we only look at $\operatorname{det}(1+Q)$ and we also forget about the power $1 / 4$, for simplicity. It only corresponds to replace $\mathrm{N} \rightarrow \mathrm{N} / 4$ in the following. (If $2 \mathrm{~N}$ does not divide by 4 the purist might treat the remaining fractional power separately...).

Applying the $\mathrm{h}$ - and $\zeta$ - derivatives to $\operatorname{det}(1+\mathrm{Q})$ reproduces a determinant structure which may also be written using antisymmetric tensor products $[13,14,15,4]$. This structure, which allows for improved estimates, traces back to the anticommuting fermionic variables or the Pauli principle.

We first regard the case where only $\mathrm{h}$ - derivatives are applied. $\zeta$ - derivatives are more difficult to describe though obeying sharper bounds. We find

$$
\begin{gathered}
\partial_{\mathrm{h}_{\mathrm{n}}} \ldots \partial_{\mathrm{h}_{1}} \operatorname{det}(1+\mathrm{Q})= \\
{\left[\mathrm{n} ! \operatorname{Tr}\left(\frac{1}{1+\mathrm{Q}} \mathrm{Q}_{1} \Lambda \ldots \Lambda \frac{1}{1+\mathrm{Q}} \mathrm{Q}_{\mathrm{n}}\right)+\operatorname{rd}\right] \operatorname{det}(1+\mathrm{Q})}
\end{gathered}
$$

with the explanations :

(i) $\Lambda$ denotes the antisymmetric tensor product

(ii) $\mathrm{Q}_{\mathrm{i}}:=\partial_{\mathrm{h}_{\mathrm{i}}} \mathrm{Q}$

(iii)rd stands for rederived terms, i.e. where $\partial_{h_{j}}, j>i$ applies again to $Q_{i}$ producing $Q_{i j}=\partial_{h_{j}} \partial_{h_{i}} Q$ etc. These terms may also be written as antisymmetric tensor products with less than $\mathrm{n}$ entries. A term as in (192) may be estimated as in [14] :

$$
\left|n ! \operatorname{Tr} \Lambda_{i=1}^{n}\left(\frac{1}{1+Q} Q_{i}\right)\right| \operatorname{det}(1+Q) \leq\left(\prod_{j=1}^{n}\left(1+\lambda_{j}\right)^{-1} \prod_{i=1}^{n} \operatorname{Tr}\left|Q_{i}\right|\right) \operatorname{det}(1+Q)
$$

where

$$
\operatorname{Tr}\left|Q_{i}\right|=\operatorname{Tr}\left(Q_{i} Q_{i}^{*}\right)^{1 / 2}
$$

and $\lambda_{\mathrm{j}}, 1 \leq \mathrm{j} \leq \mathrm{n}$, are the $\mathrm{n}$ smallest eigenvalues of $\mathrm{Q}$ (due to the antisymmetric product we do not get the $n$-th power of the smallest eigenvalue). Then we find 


$$
\prod_{j=1}^{n}\left(1+\lambda_{j}\right)^{-1} \operatorname{det}(1+Q) \leq \prod_{j=n+1}^{\infty}\left(1+\lambda_{j}\right) \leq 2^{n}
$$

(Note that $\lambda_{j}>-1$ in finite volume, but the estimate also holds on passing to the infinite volume limit). The last bound in (194) is proven on noting that

(i) we may assume without restriction $-1<\lambda_{\mathrm{i}}<0$ for $\mathrm{i} \leq \mathrm{n}$

(ii) the eigenvalues of $Q$ come in pairs $\lambda,-\lambda$ (see Lemma 16).

Now we look at the expressions for the $\mathrm{Q}_{\mathrm{i}}$. In particular we have to verify that a small factor per small field derivative (sfd) is generated. $\partial_{h}$ applied to $Q$ does not act on the term $(1+\widehat{K})^{-1}$ unless $h$ connects blocks within $S_{+}$or $S_{-}$, and it acts only on $K^{\prime}$ if $h$ connects different regions. $\sum_{a} A_{L a}$ is $h-$ independent. So if $h$ connects two $S_{+}$- squares we have

$$
\partial_{\mathrm{h}} \mathrm{Q}=\left[\partial_{\mathrm{h}}(1+\widehat{\mathrm{K}})^{-1}\right] \mathrm{K}^{\prime}=\frac{1}{1+\mathrm{K}_{+}}\left(\partial_{\mathrm{h}} \mathrm{K}_{+}\right) \frac{1}{1+\mathrm{K}_{+}} \mathrm{P}_{+} \mathrm{K}^{\prime}\left(\mathrm{P}_{-}+\mathrm{P}_{\mathrm{L}}\right)
$$

due to the support properties of $\partial_{\mathrm{h}} \mathrm{K}^{+}$and $\mathrm{K}^{\prime}$. Thus $\mathrm{K}^{\prime}$ has to bridge the gap between + and - or between + and $L_{a}$, which is at least of size $M$. The exponential decay of $K$ then produces a small factor $\mathrm{N}^{-2}=\mathrm{e}^{-\mathrm{mM}}$ through $\mathrm{K}^{\prime}$. This together with $\mathrm{O}\left(\frac{1}{\mathrm{~m}}\right) \mathrm{N}^{-2 / 5}$ from (144) leaves a net $\sim \mathrm{N}^{-4 / 3}$ on taking into account $\mathrm{N}$ from $\operatorname{Tr}\left|\mathrm{Q}_{\mathrm{i}}\right|$. If $\partial_{\mathrm{h}}$ acts on $\mathrm{K}^{\prime}$ we get the small factor directly from $\partial_{\mathrm{h}} \mathrm{K}^{\prime}$. We need however the decay of $\partial_{h} K^{\prime}$ also to sum over the (blocks of) $\mathrm{Y}$ for given tree $\mathrm{T}$. It is therefore essential that we keep a factor

$$
\mathrm{e}^{-\eta \cdot m \cdot 1(T, Y)}
$$

where $0<\eta<1$ and

$$
1(T, Y)=\sum_{(i, j)} \operatorname{dist}\left(D_{i}, D_{j}\right)
$$

the sum being over the links $(i, j)$ of the tree linking the blocks from $Y$. The simplest way of doing is thus to keep only $\mathrm{N}^{-1 / 3}$ per small field derivative and choose $\eta \leq 1 / 2$, e.g. $\eta=1 / 3$, so as not use up the decay twice. The reader should note that the factor (196) can always be extracted to wherever the derivatives apply (and always by the same reason). But we only mention it here when treating $\operatorname{det}(1+Q)$. Another fraction of the decay is used up on application of the LFP (see (190) and below). Whereas the latter comes into play for all parts of the interaction, the previous splitting is only necessary in those cases, where the small factor per sfd is generated by the large gap between 
different regions (see $\mathrm{G}_{4 \gamma} \mathrm{G}_{5 \gamma}$ above).

Now we look at the rd-terms : the (severe) restrictions on the supports of the (derived) kernels already effective in (195) will force many of these rd-terms to vanish (a fact which we do not exploit completely). Any new sfd produces a new $\mathrm{O}\left(\frac{1}{\mathrm{~m}}\right) \mathrm{N}^{-2 / 5}$ as before (and maybe further small factors). Note that there is no new $\operatorname{Tr}\left|Q_{i}\right| \sim N$ to beat! Still one realises that the number of terms, when taking into account all rd-terms, may grow as

$$
\sim \mathrm{C}^{\mathrm{n}} \mathrm{n} \text { ! }
$$

Again we prepare to use the LFP noting first

(i) the support restrictions

(ii) the exponential decay of the kernels of $\left(1+K_{ \pm}\right)^{-1}$. This decay is due to the small field condition (Lemma 13) which enforces rapid convergence of the geometric series. The decay constant is of order $\mathrm{m}-\mathrm{O}\left(\frac{1}{\mathrm{~m}}\right) \mathrm{N}^{-2 / 5}$

(iii) the kernels $\frac{1}{1+\sum_{a} A_{L a}}$ reduce to $\frac{1}{1+A_{L a}}$ if joined to some $\partial_{h} K$ with support in $L_{a}$ (to 1 otherwise).

Taking this into account one realizes that the sum over all possibilities of rederiving propagators is controlled by increasing distance factors as in (190). The situation is exactly the same as in (190) if we neglect the terms $(1+\widehat{K})^{-1}$ in a first step. Rederiving also those we even get a factorially increasing number of non-vanishing contributions when the coordination numbers of $T$ do not grow large. Then (ii), (iii) come into play : If $\left(\partial_{\mathrm{h}_{\mathrm{i}+1}} \mathrm{~K}_{( \pm)}^{(\mathcal{)})}\right.$ is to be grouped together with $\partial_{\mathrm{h}_{1}} \mathrm{~K}_{( \pm)}^{\left({ }^{(}\right)}, \ldots, \partial_{\mathrm{h}_{\mathrm{i}}} \mathrm{K}_{( \pm)}^{\left.()^{(}\right)}$from previous derivatives, its support has to be more and more distant from that of most of the $\partial_{\mathrm{h}_{1}} \mathrm{~K}_{( \pm)}^{\left({ }^{\prime}\right)}, \ldots \partial_{\mathrm{h}_{\mathrm{i}}} \mathrm{K}_{( \pm)}^{(\mathcal{)})}$ from previous derivatives for $\mathrm{i}$ large, and we get again an estimate as in (190).

We shortly mention that derivatives linking to lfs and thus applying to $\mathrm{K}^{\prime}$ may generate large numbers of $\zeta$-fields in lfs. These are estimated with the help of (129), or with (136) and the LFP, if they accumulate in a single block. A finer analysis would also display improved estimates due to the inverted square roots, compensating effects of these fields.

The last point to mention concerns the complications caused by the $\zeta$ - derivatives. They give a smaller contribution per sfd, since $\mathrm{O}\left(\frac{1}{\mathrm{~m}}\right) \mathrm{N}^{-2 / 5}$ may be replaced by $\mathrm{g} \sim \mathrm{N}^{-1 / 2}$. The complication comes again from the fact that they are attached to the derived covariance $\partial_{\mathrm{h}} \mathrm{C}_{\gamma}^{\mathrm{Y}}$ (see (170)). Since

$$
\partial_{\mathrm{h}_{\mathrm{i}}} \mathrm{C}^{(\gamma)}=\left(\frac{\partial \mathrm{S}}{\partial_{\mathrm{h}_{\mathrm{i}}}}\right) \widehat{\mathrm{C}}_{\gamma} \mathrm{S}(\mathrm{h})+\mathrm{S}(\mathrm{h}) \widehat{\mathrm{C}}_{\gamma} \frac{\partial \mathrm{S}}{\partial \mathrm{h}_{\mathrm{i}}}
$$


(restricted to the polymer $\mathrm{Y}$ in question) we get two terms if the derivative is associated to large field blocks, i.e. to the suppressed region. Regarding e.g. the first we thus have to replace the kernels $Q_{i}(y, x)$ from (192) by

$$
\widehat{Q}_{i}\left(y, x, z_{i}\right)=\int \frac{\delta Q(y, x)}{\delta \zeta\left(y^{\prime}\right)} \frac{\partial S}{\partial h}\left(y^{\prime}, z_{i}\right) d^{2} y^{\prime}
$$

The kernel $\left(\widehat{C}_{\gamma} S(h)\right)\left(z_{i}, w\right)$ is then taken out of the respective tensor product which now depends on the parameter $z_{i}$. The integration over the $z_{i}$ may be performed later using the exponential decay of $S(h)$. The second $\zeta$-derivative $\frac{\delta}{\delta \zeta(w)}$ may apply again to $\operatorname{det}(1+Q)$ or to any other $G_{i \gamma}$ a fact which admittedly makes it hard to present the expansion terms by explicit expressions. This does not alter the bounds, however, or change the mechanisms by which we control the expansion and which were described when treating the $\mathrm{h}$ - derivatives.

To resume we get from deriving $\operatorname{det}(1+\mathrm{Q}) \mathrm{n}$ times an expression which may be bounded by

(i) a factor $\leq \mathrm{O}(1 / \mathrm{m}) \mathrm{N}^{-2 / 5}$ per sfd

(ii) a factor $\mathrm{e}^{-\eta^{\prime} \mathrm{m} l(\mathrm{~T})}, \eta^{\prime} \leq 1 / 2$, e.g. $\eta^{\prime}=1 / 4$.

(iii) a (large) constant $\left(\mathrm{O}\left(\mathrm{m}^{-2}\right)\right)^{\mathrm{n}}$ generated by the integrations over kernels and from the LFP bounds and which also contains the combinatoric and all other $m$-independent constants. $(\zeta$ - fields from the V-region generated by derivatives are also included in this bound once they have been integrated with the aid of (136)).

(iv) a factor of $1+\delta(\delta<<1)$ per sfd and a factor of $1+\mathrm{O}\left(\mathrm{m}^{2} \mid \mathrm{L}_{\mathrm{a}} \mathrm{l}\right)$ per lfd.

These factors come from bounding the terms generated by $\left(1+\mathrm{K}_{+}+\mathrm{K}_{-}+\sum_{\mathrm{a}} \mathrm{A}_{\mathrm{La}}\right)^{-1}$ on iterated application of derivatives, taking into account support restrictions (see above (195)). The bound on the operator norm $\left\|\left(1+\mathrm{K}_{ \pm}\right)^{-1}\right\|$ follows from the small field condition, the other one from

Lemma $17: 0 \leq\left(1+A_{L a}\right)^{-1} \leq 1+O\left(m^{2}\left|L_{a}\right|\right)$ in the operator sense on $\mathfrak{L}^{2}\left(L_{a}\right)$

Proof: We have

$$
\begin{aligned}
1+A_{L a}=1+g^{2} & \sigma_{L a} \frac{1}{p^{2}+m^{2}} \sigma_{L a}-P_{L a} \frac{m^{2}}{p^{2}+m^{2}} P_{L a} \geq \\
& \geq 1-P_{L a} \frac{m^{2}}{p^{2}+m^{2}} P_{L a}
\end{aligned}
$$

now one verifies 


$$
\mathrm{p}^{2}+\mathrm{m}^{2} \geq \mathrm{m}^{2}+\left.\mathrm{O}(1) \mathrm{IL}_{\mathrm{a}}\right|^{-1}
$$

on $\mathrm{E}$, where $\mathrm{E}$ is the space of smooth functions with support in $\mathrm{L}_{\mathrm{a}} \mid \partial \mathrm{L}_{\mathrm{a}} . \mathrm{E}$ is dense in $\mathfrak{J}^{2}\left(\mathrm{~L}_{\mathrm{a}}\right)$ as is

$$
\begin{gathered}
\widetilde{E}:=\left(p^{2}+m^{2}\right)(E), \\
\text { and on } \widetilde{E}(\text { from }(200)) \\
\frac{1}{p^{2}+m^{2}} \leq \frac{1}{m^{2}+O(1) \mid L_{a}{ }^{-1}}
\end{gathered}
$$

which then holds by continuity on $\mathfrak{L}^{2}\left(\mathrm{~L}_{\mathrm{a}}\right)$.

Therefore

$$
\mathrm{P}_{\mathrm{La}} \frac{\mathrm{m}^{2}}{\mathrm{p}^{2}+\mathrm{m}^{2}} \mathrm{P}_{\mathrm{La}} \leq \frac{\mathrm{m}^{2}}{\mathrm{~m}^{2}+\left.\mathrm{O}(1) \mathrm{L}_{\mathrm{a}}\right|^{-1}}
$$

and

$$
\left(1+A_{L_{a}}\right)^{-1} \leq\left(1-\frac{m^{2}}{m^{2}+O(1)\left|L_{a}\right|^{-1}}\right)^{-1}=1+O\left(m^{2}\left|L_{a}\right|\right)
$$

For (very) large $\left|\mathrm{L}_{\mathrm{a}}\right|$ these factors are easily controlled by (135), (136).

QED

$\operatorname{det}_{3}^{1 / 2}\left(1+K_{ \pm}\right):$

The essential modification comes from the det $_{3}$. Taking one $\mathrm{h}$ - derivative we obtain

$$
\partial_{\mathrm{h}} \operatorname{det}_{3}(1+\mathrm{K})=\operatorname{Tr}\left(\frac{1}{1+\mathrm{K}} \mathrm{K}^{2} \partial_{\mathrm{h}} \mathrm{K}\right) \operatorname{det}_{3}(1+\mathrm{K})
$$

Furthermore $n$ derivatives produce

$$
\left\{\mathrm{n} ! \operatorname{Tr}\left(\frac{1}{1+\mathrm{K}}\left(\mathrm{K}^{2} \partial_{\mathrm{h}_{1}} \mathrm{~K}\right) \Lambda \ldots \Lambda \frac{1}{1+\mathrm{K}}\left(\mathrm{K}^{2} \partial_{\mathrm{h}_{\mathrm{n}}} \mathrm{K}\right)\right)+\mathrm{rt}\right\} \operatorname{det}_{3}(1+\mathrm{K})
$$

to be compared to (192) (see also [4]).

So the essential modification is the replacement $\partial_{h} K \rightarrow K^{2} \partial_{h} K$. The small factor per sfd is now again $\mathrm{O}\left(\mathrm{m}^{-3}\right) \mathrm{N}^{-1 / 5}$ (see (158), (192)) $\left(\zeta\right.$ - derivatives produce an additional $\mathrm{N}^{-1 / 10}$ ). Note that the small factor would not have been produced on expanding det or $\operatorname{det}_{2}$ but comes from the third 
power of $\mathrm{K}$, each power contributing $\mathrm{N}^{-2 / 5}$. The remaining terms ' $r$ t' $^{\prime}$ from (201) either stem from rederiving the $\mathrm{K}^{2} \partial_{\mathrm{h}} \mathrm{K}$ which is now possible at most once with respect to $\mathrm{h}$ (using support restrictions) or five times with respect to $\zeta$. Each $\zeta$ - derivative produces a new $\mathrm{N}^{-1 / 10}$ whereas a second $\partial_{\mathrm{h}_{2}}$ applied to $\mathrm{K}^{2} \partial_{\mathrm{h}_{1}} \mathrm{~K}$ does not bring down a new small factor (unless it vanishes). Thus in the worst case we only get

$$
\mathrm{O}\left(\mathrm{m}^{-3}\right) \mathrm{N}^{-1 / 5}=\mathrm{o}\left(\mathrm{N}^{-1 / 10}\right)
$$

per $2 \mathrm{~h}$ - derivatives. These terms, however, are not accompanied by large combinatoric factors and have additional distance decay due to the trace conditions.

Among the rt-terms we also count the correction terms appearing when we derive again $(1+K)^{-1}$ and then want to bring the result back into the form $(1+K)^{-1} K^{2} \partial_{h} K$, e.g. :

$$
\begin{gathered}
\partial_{\mathrm{h}_{2}} \operatorname{Tr}\left(\frac{1}{1+\mathrm{K}} \mathrm{K}^{2}\left(\partial_{\mathrm{h}_{1}} \mathrm{~K}\right)\right)=-\operatorname{Tr}\left(\frac{1}{1+\mathrm{K}} \partial_{\mathrm{h}_{2}} \mathrm{~K} \frac{1}{1+\mathrm{K}}\left(\mathrm{K}^{2} \partial_{\mathrm{h}_{1}} \mathrm{~K}\right)\right)+\ldots \\
\left.=-\operatorname{Tr}\left(\frac{1}{1+\mathrm{K}} \mathrm{K}^{2}\left(\partial_{\mathrm{h}_{2}} \mathrm{~K}\right) \frac{1}{1+\mathrm{K}} \mathrm{K}^{2} \partial_{\mathrm{h}_{1}} \mathrm{~K}\right)-\operatorname{Tr}\left(\partial_{\mathrm{h}_{2}} \mathrm{~K}\right) \frac{1}{1+\mathrm{K}} \mathrm{K}^{2} \partial_{\mathrm{h}_{1}} \mathrm{~K}\right) \\
+\operatorname{Tr}\left(\mathrm{K}\left(\partial_{\mathrm{h}_{2}} \mathrm{~K}\right) \frac{1}{1+\mathrm{K}} \mathrm{K}^{2} \partial_{\mathrm{h}_{1}} \mathrm{~K}\right)+\ldots
\end{gathered}
$$

The first term is of appropriate shape to be grouped together with the term where the derivative applies to $\operatorname{det}_{3}(1+\mathrm{K})$. The other terms are estimated separately and contain one or two supplementary $\mathrm{O}\left(\mathrm{N}^{-2 / 5}\right)$.

We note that it is not really necessary to perform this regrouping of terms and to use the antisymmetric tensor product structure here, since (the kernel of) $(1+K)^{-1}$ is bounded (and exponentially decreasing) in the small field domain. So the LFP is sufficient to estimate the sum of all terms. The rest of the discussion is analogous as (but somewhat simpler than) that of det $(1+Q)$. So we stop here.

$\operatorname{det}_{2}{ }^{1 / 2}\left(1+A_{L a}\right):$

The treatment is as before, but the $\mathrm{A}_{\mathrm{La}}$ are independent of $\mathrm{h}$ - parameters. $\zeta$ - derivatives produce antisymmetric tensor products of

$$
\frac{1}{1+\mathrm{A}_{\mathrm{La}}} \mathrm{A}_{\mathrm{La}} \frac{\delta \mathrm{A}_{\mathrm{La}}}{\delta \zeta}
$$


joined to $\partial_{\mathrm{h}} \mathrm{C}_{\gamma}^{Y}$ 's, which always have to bridge a large gap, producing an $\mathrm{N}^{-2}$, which we do not really need, however, in the large field domain. One $A_{\mathrm{La}} \frac{\delta A_{\mathrm{La}}}{\delta \zeta}$ may be rederived at most three times any rederivation being accompanied again by a negative power of $\mathrm{N}$ through the distance gaps. Lemma 17 can be used to bound $\left(1+\mathrm{A}_{\mathrm{La}}\right)^{-1}$ as before. 


\section{IV.4. Results}

Now we come to the

\section{Proof of Proposition 3 :}

We have to bound $\mathrm{a}\left(\mathrm{Y}^{\ell}\right)$ for given $\mathrm{Y}^{\ell}$ consisting of $\mathrm{n}_{\mathrm{s}}$ small field blocks $\Delta_{\mathrm{j}}$ and of $\mathrm{n}$ large field blocks $\mathrm{D}_{\mathrm{i}}$ with

$$
\cup D_{i}=\Gamma \subset Y, \cup \Delta_{j}=S \subset Y, Y=S \cup \Gamma
$$

(i.e. all regions are restricted to $\left.\mathrm{Y}=\mathrm{Y}^{\ell}\right)$. From the previous section $\mathrm{a}\left(\mathrm{Y}^{\ell}\right)$ is bounded by a product of factors :

(1) (i) a small factor $\leq\left(\mathrm{m}^{-3} \mathrm{~N}^{1 / 5}\right)^{\mathrm{n}_{\mathrm{S}} / 2}$ (see (202))

(ii) $\exp \left(\mathrm{O}(1) \mathrm{N}^{-1 / 10} \mathrm{n}_{\mathrm{s}}\right.$ ) (see Proposition 2, Corollary 1) from the small field region.

(2) $\mathrm{a}\left(\mathrm{Y}^{\ell}\right)$ contains a sum over trees $\mathrm{T}$. For given tree we have in the bound a factor

$$
\exp (-\eta \mathrm{m} l(\mathrm{~T}, \mathrm{Y})) \quad \text { with (e.g.) } \eta=1 / 3
$$

(see (169), (171), (196)).

(3) In the large field region we get a bound

$$
\exp \left\{-\frac{b}{2}|\Gamma| N^{1 / 5}\right\}
$$

which may be deduced as follows :

From Prop. 2 and (136) we have a bound in the large field domain of

$$
\begin{aligned}
& \exp \left(-\mathrm{b}|\Gamma| N^{1 / 5}+(\ln 2)\right)|\gamma| N^{1 / 10}-\frac{1}{100}|\mathrm{~V}| \mathrm{N}+ \\
& \left.\left.+\mathrm{O}\left(\frac{1}{\mathrm{~N}}\right)\left(|\mathrm{L}|\left(\left\|\zeta_{\mathrm{L}}\right\|+1\right)+\left\|\zeta_{\mathrm{L}}\right\|^{2}\right)-\frac{1}{60}\left\|\zeta_{\mathrm{v}}\right\|^{2}\right)\right\}
\end{aligned}
$$

Here we used only a fraction of (136). The rest is used to integrate and then bound the contribution of the $\zeta$ - fields descended by derivatives in the $\mathrm{V}$ - region. $\zeta$ - fields descended in $\mathrm{l} V \mathrm{~V}$ region are 
bounded by (129). From those and from Lemma 17 we get factors $\sim \mathrm{O}\left(\mathrm{IL}_{\mathrm{a}} \mathrm{l}\right)$ per lfd. All those contributions are easily incorporated in (205) since we have replaced $b \rightarrow \frac{b}{2}$.

(4) Finally we have a product of $R_{1}{ }^{n_{s}} R_{2}{ }^{{ } \ell}$ with $N$-independent constants $R_{1}, R_{2}$. These terms take care of all combinatoric factors from the choices onto which $G_{i \gamma}$ to apply the $h$ - and $\zeta$ - derivatives and how to apply them within each $\mathrm{G}_{\mathrm{i} \gamma}$. They take into account the negative powers of $\mathrm{m}^{2}$, generated by applying the LFP in its possible forms. This also includes the integration of the descended $\zeta$ - fields in the (highly suppressed) V - region. Finally estimates of the type (194) and the bounds on the kernels in Lemmas 5 and 7 contribute to $R_{1,2}$. Just taking maximal values everywhere leads to tremendous constants $\mathrm{R}_{1}, \mathrm{R}_{2}$. For simplicity and laziness we do not optimize them. Note, however that any constant generated per lfd can be easily absorbed in the strong bound (205), contributions from $\mathrm{V}$ are really tiny. Finally putting $\mathrm{m}^{3 / 2} \mathrm{~N}^{-1 / 10}$ per sfd in (1) corresponds to the worst case, appearing only in (202) for a single choice of applying the derivatives (and which for many trees is only allowed much less than $n_{s}$ times). All other choices imply additional suppression factors of $\mathrm{N}^{-1 / 20}, \mathrm{~N}^{-1 / 10}, \mathrm{~N}^{-1 / 2}, \ldots$ per derivative which then beat the contributions to $\mathrm{R}_{1}{ }^{\mathrm{n}_{\mathrm{s}}}$ completely.

To pass from $\mathrm{a}(\mathrm{Y} \ell)$ to $\mathrm{b}(\mathrm{Y})$ we have to sum over the admitted assignments $\ell, \mathrm{s}_{ \pm}$for $\mathrm{Y}$. By bold overestimation this leads to an additional $3^{|Y|}$. More carefully, one may take into account the stringent restrictions on the possible assignments and the strong suppression in the large field region to absorb the large field choices in (205) to (essentially) eliminate this factor.

Collecting everything we thus find

$$
\begin{gathered}
\left|\sum_{0 \in \mathrm{Y} \subset \Lambda} b(\mathrm{Y}) \mathrm{e}^{|\mathrm{Y}|}\right| \leq \\
\leq 1 \sum_{\mathrm{O} \in \mathrm{Y}^{\mathrm{I}} \subset \Lambda, \ell, \mathrm{s}_{+} \mathrm{T}} \sum_{\mathrm{T}} \mathrm{e}^{-1 / 3 \mathrm{~m} \mathrm{l}\left(\mathrm{T}, \mathrm{Y}^{\ell}\right)}(\mathrm{eR})^{|\mathrm{Y}|}\left(\mathrm{m}^{-\frac{3}{2}} \mathrm{~N}^{-\frac{1}{10}}\right)^{\mathrm{n}_{\mathrm{s}}} \exp \left(-\frac{\mathrm{b}}{2}|\Gamma| \mathrm{N}^{1 / 5}\right) \leq \\
\leq \sum_{\mathrm{n} \geq 2}\left(\mathrm{R}^{\prime} \mathrm{N}^{-} \frac{1}{10} \mathrm{~m}^{-\frac{7}{2}}\right)^{\mathrm{n}} \leq 1 / 2
\end{gathered}
$$

for $\mathrm{N}$ sufficiently large, with the above remarks on $\mathrm{R}\left(\sim \mathrm{R}_{1}\right)$ and $\mathrm{R}^{\prime}=\mathrm{O}(1)$ e $\mathrm{R}$. The sum over the trees $\mathrm{T}$ is performed as in [9], Lemma III. 1.4, using Cayley's theorem.

QED

As noted in section IV.2, the bound of Prop. 3 is sufficient to prove the convergence of the series (184) for $S_{2}\left(f_{1}, f_{2}\right)$ in the infinite volume limit. Our aim is now to prove also that this series decays exponentially with the distance of the supports of $f_{1}$ and $f_{2}$. 
Our main result is

Theorem : The infinite volume two point function decays exponentially :

$$
\left|S_{2}\left(f_{1}, f_{2}\right)\right| \leq O(1) \exp \left\{-m^{\prime} \operatorname{dist}\left(f_{1}, f_{2}\right)\right\}
$$

where

$$
m^{\prime}=m\left(1+o\left(N^{-1 / 10}\right)\right)
$$

dist $\left(f_{1}, f_{2}\right)=\inf \left\{\left|x_{1}-x_{2}\right| ; x_{1,2} \in \operatorname{supp} f_{1,2}\right\} . O(1)$ is an $N$ - independent number. The estimate on $\mathrm{m}^{\prime}$ is (of course) not optimal.

Proof: When treating external polymers we have to apply the cluster expansion also to

$$
F_{2}(\zeta)=<f_{1}, \frac{1}{p+g \sigma} f_{2}>
$$

There is a slight technical nuisance with this term, since the BBF - expansion as applied here requires the expanded kernels to be symmetric in $(x, y)$.

Remembering that $1+K=\left(1+g \tau \frac{1}{-p+m}\right)\left(1+\frac{1}{p+m} g \tau\right)(24)$, we therefore write :

$(210)<f_{1}, \frac{1}{p+g \sigma} f_{2}>=<r, \frac{1}{1+K} f_{2}>+<s(\tau), \frac{1}{1+K} f_{2}>$

where

$$
\mathrm{r}=\frac{1}{-p+\mathrm{m}} \mathrm{f}_{1}, \mathrm{~s}(\tau)=\frac{1}{p+\mathrm{m}} \mathrm{g} \tau \frac{1}{-p+\mathrm{m}} \mathrm{f}_{1}
$$

so that (210) equals :

$$
\underset{\Delta^{\prime}}{\sum}<\mathrm{r}_{\Delta^{\prime}}, \frac{1}{1+\mathrm{K}} \mathrm{f}_{2}>+\sum_{\Delta^{\prime} \Delta^{\prime \prime}}<\mathrm{s}_{\Delta^{\prime \prime}}\left(\tau_{\Delta^{\prime}}\right), \frac{1}{1+\mathrm{K}} \mathrm{f}_{2}>
$$

with

$$
\mathrm{r}_{\Delta^{\prime}}=\chi_{\Delta^{\prime}} \frac{1}{-\not p+m} \mathrm{f}_{1}, \mathrm{~s}_{\Delta^{\prime \prime}}\left(\tau_{\Delta^{\prime}}\right)=\chi_{\Delta^{\prime \prime}} \frac{1}{\not p+m} \mathrm{~g} \tau_{\Delta^{\prime}} \frac{1}{-\not p+m} \mathrm{f}_{1}
$$

and the sums are over all squares which form $\Lambda$.

From the exponential decay of $( \pm p+m)^{-1}$ (and the established estimates to bound $\tau_{\Delta^{\prime}}$ ) it is then 
obvious that the Theorem holds for $F_{2}(\zeta)$ if it holds on replacing $F_{2}$ by

$$
\widetilde{F}(\zeta)=\left\langle f_{1}, \frac{1}{1+K} f_{2}>\right.
$$

since the sums in (211) are easily estimated. So we replace $F_{2} \rightarrow \widetilde{F}$ in the following. As we know from sect. IV.3, $1+\mathrm{K}$ may approach 0 for large volume. We therefore group together

$$
\widetilde{\mathrm{F}}(\zeta) \operatorname{det}_{2}{ }^{1 / N}(1+\mathrm{K})=: \widetilde{\mathrm{F}}(\zeta) \operatorname{det}_{2}(1+\mathrm{k})
$$

where $\operatorname{det}_{2}{ }^{1 / N}(1+K)$ may be isolated from (53), thereby replacing $N \rightarrow N-2$ in the following. The power $1 / \mathrm{N}$ just means that we do not take a flavour trace. For an external polymer we now get also contributions from applying derivatives to (213). The treatment is analogous to that of the determinants in sect. IV.3. But the contributions due to sfd are now much smaller since we do not have a factor $\mathrm{N}$ from the traces to beat. Bounding the expression analogous to (192) we now find instead of (194) (see also [14, 15])

$$
\begin{gathered}
\left|\prod_{i=1}^{n}\left(1+\kappa_{i}\right)^{-1} \operatorname{det}_{2}(1+k)\right| \leq \prod_{i=n+1}^{\infty}\left(1+\kappa_{i}\right) e^{-\sum_{i=1}^{\infty} \kappa_{i}} \\
\quad=\exp \sum_{i=n+1}^{\infty}\left(\ln \left(1+\kappa_{i}\right)-\kappa_{i}\right) e^{-\sum_{i=1}^{n} \kappa_{i}} \leq e^{n}
\end{gathered}
$$

where $\kappa_{i}$ are the eigenvalues of $\kappa(\zeta)$, ordered as $\kappa_{1} \leq \kappa_{2} \leq \ldots$, and we used $\kappa>-1$ (in finite volume).

To prove exponential decay we have to look at the external polymer $Y$ contained in $\mathrm{b}^{\mathrm{T}}(\mathrm{M})$ (184). We have $\Delta\left(f_{1}\right), \Delta\left(f_{2}\right) \subset Y$, where $\Delta\left(f_{1}\right), \Delta\left(f_{2}\right)$ contain the supports of $f_{1}, f_{2}$. By definition of $Y$ there is for any tree $\mathrm{T}$ a sequence of links joining the two blocks which contain these supports (in any assignment $\mathrm{Y}^{\ell, s_{+}}$of $\mathrm{Y}$ ).

We first suppose $Y$ to be assigned such that $\ell$ is empty, which is the dominant contribution. The problem consists in putting aside the distance decay between $\Delta\left(\mathrm{f}_{1}\right), \Delta\left(\mathrm{f}_{2}\right)$ without invalidating the convergence proof. We proceed as follows :

For any tree $\mathrm{T}$ we split up

$$
\mathrm{T}=\mathrm{T}^{\prime} \cup \mathrm{T}^{\prime \prime},
$$

where $T^{\prime}$ is a minimal number of links in $T$ joining $\Delta\left(f_{1}\right), \Delta\left(f_{2}\right)$ (no matter which). Thus the tree $T^{\prime}$ 
has at least one link, and all of its coordination numbers $d_{i}^{\prime}$ fulfill $: d_{i}^{\prime} \leq 2$. Corresponding to $T^{\prime}$ we put aside a factor

$$
\varepsilon^{\left|T^{\top}\right|} \prod_{l^{\prime} \in T^{*}} P_{1} \cdot\left(x_{1^{\prime}}, y_{1^{\prime}}\right)
$$

where | $\mathrm{T}^{\prime}$ l contains the number of links $\mathrm{l}^{\prime}$ in $\mathrm{T}^{\prime}$, and $\varepsilon=\mathrm{O}(1) \mathrm{m}^{-3} \mathrm{~N}^{-1 / 5}=\mathrm{o}\left(\mathrm{N}^{-1 / 10}\right)$. (One realizes that the special choice of derivatives (202) does not have to be taken into account here. Otherwise we had to replace $\left.\varepsilon \rightarrow \varepsilon^{1 / 2}\right) . \mathrm{P}_{\mathrm{l}^{\prime}}\left(\mathrm{x}_{\mathrm{l}}, \mathrm{y}_{\mathrm{l}}\right)$ is a shorthand notation for the kernels associated to the links $\mathrm{l}^{\prime} \in \mathrm{T}^{\prime}$ and generated by the respective derivatives. All these kernels decay at least exponentially with constant $\mathrm{m}$ (even with $\mathrm{m}+\mathrm{O}(1) \mathrm{m}^{2} \mathrm{l}$ ) according to Lemma 5 . And so the product in (216) is bounded in modulus by

$$
\varepsilon^{\left|T^{\prime \prime}\right|} \prod_{l \in T^{\prime}}\left(\frac{1}{p^{2}+m^{2}}\right)\left(\operatorname{dist}\left(\Delta_{a}\left(l^{\prime}\right), \Delta_{l^{\prime}}\right)\right) \leq(O(1) \varepsilon)^{\left|T^{\prime \prime}\right|} \prod_{l \in T^{\prime}} e^{-m \operatorname{dist}\left(\Delta_{a}\left(l^{\prime}\right), \Delta^{\prime}\right)}
$$

where $\Delta_{\mathrm{a}\left(\mathrm{l}^{\prime}\right)}, \Delta_{\mathrm{l}}$ are the squares linked by $\mathrm{l}^{\prime}$, and we used the kernel of $\left(\mathrm{p}^{2}+\mathrm{m}^{2}\right)^{-1}:\left(\frac{1}{\mathrm{p}^{2}+\mathrm{m}^{2}}\right)(\mathrm{x}-\mathrm{y})$. For shortness we call $\mathrm{a}^{\prime}\left(\mathrm{Y}, \mathrm{T}, \mathrm{T}^{\prime}\right)$ the remainder of the amplitude of $\mathrm{Y}$ for given $\mathrm{T}$.

The important point is that the LFP may still be used to bound the remainder of $a(Y, T)$ without using up (217) : the LFP is only needed (in a small field polymer), when the tree $T$ has large coordination numbers $d_{j}>1$. But since $d_{i}{ }^{\prime} \leq 2$, the coordination numbers of $T$ " fulfill $d_{j}{ }^{\prime \prime} \geq d_{j}-2$, and the reduction by at most 2 is unimportant in (190). So the LFP works as before. We estimate

$$
\left|\sum_{T} \sum_{Y} a(Y, T)\right| \leq \sum_{T, Y} \varepsilon^{\left|T^{\prime \prime}\right|} \sup _{\substack{x \in \Delta\left(f_{1}\right), y \in \Delta\left(f_{2}\right)}}\left(\frac{1}{p^{2}+m^{2}}\right)^{\left|T^{\prime \prime}\right|}(x-y)\left|a^{\prime}\left(Y, T, T^{\prime}\right)\right|
$$

$\mathrm{a}^{\prime}\left(\mathrm{Y}, \mathrm{T}, \mathrm{T}^{*}\right)$ is the rest of $\mathrm{a}(\mathrm{Y}, \mathrm{T})$ after taking out the $\mathrm{P}_{1}$. It thus includes also all choices as to which kernel $P_{1}$ may be. All these kernels are bounded in modulus by the one of $\left(\mathrm{p}^{2}+\mathrm{m}^{2}\right)^{-1}$ (Lemmas 5,7). Finally we used in (218) the pointwise positivity of the kernels of $\left(\mathrm{p}^{2}+\mathrm{m}^{2}\right)^{-\mathrm{n}}$ to estimate for any $\mathrm{O}$ $\subset \mathbb{R}^{2}$

$$
\int_{O}\left(\frac{1}{p^{2}+m^{2}}\right)(x-y)\left[\left(\frac{1}{p^{2}+m^{2}}\right)^{n}\right](y-z) d^{2} y \leq\left[\left(\frac{1}{p^{2}+m^{2}}\right)^{n+1}\right](x-z)
$$

etc.

Using (219) the kernel in (218) has already been freed from the positions of the intermediate squares between $\Delta\left(f_{1}\right), \Delta\left(f_{2}\right)$. 
When summing over |T'| we obtain for (218) the estimate

$$
\left(\sum \sup _{T, Y} \mid a^{\prime}(Y) T, T^{\prime}\right) \mid \sum_{n \geq 1} \sup _{\substack{x \in \Delta\left(f_{1}\right), y \in \Delta\left(f_{2}\right)}}\left(\frac{\varepsilon}{p^{2}+m^{2}}\right)^{n}(x-y),
$$

and the right sum satisfies by geometric expansion and pointwise positivity

$$
\begin{aligned}
& \sup _{\substack{x \in \Delta\left(f_{1}\right), y \in \Delta\left(f_{2}\right)}}\left(\frac{1}{p^{2}+m^{2}-\varepsilon}-\frac{1}{p^{2}+m^{2}}\right)(x-y) \leq \\
& \leq O(\varepsilon) \sup _{\substack{x \in \Delta\left(f_{1}\right), y \in \Delta\left(f_{2}\right)}} \exp \left\{-m\left(1-\frac{\varepsilon}{m}\right)(x-y)\right\}
\end{aligned}
$$

(When taking into account the effect of the UV regularization we may replace $m \rightarrow m\left(1+\left|O(1) \mathrm{m}^{2}\right|\right)$. The bound on the first sum in (220) is then achieved as that of Proposition 3, by our previous discussion - with one more explanation : if any of the $\Delta_{\mathrm{a}\left(\mathrm{l}^{\prime}\right)}, \Delta_{\mathrm{l}}$, linked by l' $\in \mathrm{T}^{\prime}$, appears only in links of $\mathrm{T}^{\prime}$, but not in $\mathrm{T}^{\prime \prime}$ - so that no decay is available to sum over its position in the first sum in (220), then we do not sum over its position in (220): this sum has already been taken account of by the bound (218). Instead of summing we only take the sup over all possible squares. (If there are additional links we may or may not sum).

It is of course not true that $S_{2}$ vanishes for $\varepsilon \rightarrow 0(\mathrm{~N} \rightarrow \infty)$. The dominant contribution comes from (211)

This contributes

$$
\sum_{\Delta^{\prime}}<\mathrm{r}_{\Delta^{\prime}}, \frac{1}{1+\mathrm{K}} \mathrm{f}_{2}>\text { for } \mathrm{Y}=\Delta\left(\mathrm{f}_{2}\right)=\Delta^{\prime}
$$

$$
<f_{1} \frac{1}{p+m}\left(\frac{1}{1+K}\right)_{Y} f_{2}>=<f_{1}, \frac{1}{p+m} f_{2}>\left(1+O\left(N^{-2 / 5}\right)\right) .
$$

Finally we consider the case when $Y$ contains also large field blocks. $\Delta\left(f_{1}\right), \Delta\left(f_{2}\right)$ may now be contained in two blocks $D_{1} \neq D_{2}$, where at least one is large field, or in a single large field block $D$. For large blocks

$$
\sup _{\substack{x \in D_{1} \\ y \in D_{2}}}\left(\frac{1}{p^{2}+m^{2}}\right)^{n}(x-y)
$$


or

$$
\sup _{x, y \in D}\left(\frac{1}{p^{2}+m^{2}}\right)(x-y)
$$

need not decrease. But in this case we may take the missing decay (and much more) from

$$
\exp \left(-\frac{b}{2}\left|D_{i}\right| N^{1 / 5}\right), \exp \left(-\frac{b}{2}|D| N^{1 / 5}\right)
$$

i.e. from the large field suppression. The large field decay also is (more than) sufficient to make the LFP work in the situations which only appear in large field polymers (sect. IV.3). The essential reason that large field polymers do not pose any problem, is in the fact that the large field suppression exponential has not only a much larger coefficient than $\mathrm{m}$, but is also proportional to $|\Gamma|$, and $|\Gamma| \gg>$ sup dist $(x-y)$. So we stop here.

$$
x, y \in \Gamma
$$

After extracting the exponential decay from a $(\mathrm{Y}, \mathrm{T})$ the convergence proof for (184) follows from Proposition 3 as in [9], ch. III. 1.

\section{QED}

We finish with the promised statement on the expectation value of $\sigma$.

Proposition 4: $\left\langle\sigma_{\Delta}\right\rangle=\frac{\mathrm{m}}{\mathrm{g}}\left(1+\mathrm{o}\left(\mathrm{N}^{-1 / 20}\right)\right)$.

Proof: We proceed as before. An external polymer is now one containing the (fixed) square $\Delta$. If $\Delta$ is in $s_{+}$and $Y$ consists of $\Delta$ only, we have for the amplitude (see (160))

$$
\mathrm{a}(\Delta)=\frac{\mathrm{m}}{\mathrm{g}}\left(1+\mathrm{o}\left(\mathrm{N}^{-1 / 10}\right)\right.
$$

since $\sigma_{\Delta}=\left(\frac{\mathrm{m}}{\mathrm{g}}\right)_{\Delta}+\zeta_{\Delta}$, and $\left\langle\zeta_{\Delta}>\sim \mathrm{O}(1)\right.$ in $\mathrm{s}_{+}$.

For larger polymers we may gain as before o $\left(\mathrm{N}^{-1 / 20}\right)$ per sfd, large field polymers are exponentially suppressed. The crucial point is the following : If $\Delta \in \mathrm{s}_{-}$, the boundary conditions imply $\Gamma \neq \varnothing$. So this assignment, which potentially would give $\sim-\frac{\mathrm{m}}{\mathrm{g}}$, is always exponentially suppressed. 


\section{Conclusions.}

The Gross- Neveu - Model plays a prominent role in the program of constructive field theory. The reason is that is shares two important features with four dimensional gauge theories, though being technically much simpler. These are UV asymptotic freedom and a nonperturbative mechanism of mass generation. Gauge theories could so far only be studied in the UV regime by constructive methods (and this only near the edge of the region where technicalities become prohibitive) [16]. This paper starts the analysis of the Gross - Neveu - Model with discrete chiral symmetry from the IR - viewpoint. Constructive methods presently require a small expansion parameter which is here $\frac{1}{\mathrm{~N}}$ and which has to be unfortunately unrealistically small, but nevertheless finite! We prove the exponential decay of the two-point function and the existence of (at least) two pure phases.

As regards the UV part of the problem, the massive Gross-Neveu-Model (on introducing a bare mass by hand - also called Mitter-Weisz-Model [17]-) has not only been constructed for any $\mathrm{N}>2$ and small renormalized coupling $[2,3]$, but also analyzed in more detail. Thus the Wilson Zimmermann short - distance expansion and asymptotic completeness in the two-particle region have been established [18]. For (again very) large $N$ the UV analysis even includes the perturbatively nonrenormalizable three-dimensional model [4]. As regards the future, it remains to close the gap between the UV and IR constructions by taking away the UV cutoff from the latter. We also intend to analyze the model with continuous chiral symmetry whose infrared structure is certainly richer and more complicated. To go even further one would hope to get a complete picture of the phase structure of the large $\mathrm{N}$ models and to make contact with the results obtained using the so-called complete integrability of the Gross-Neveu Model. Theses results while based on a certain number of unproven assumptions provide a riche amount of information [19]. From the technical point of view the methods are completely separate from ours.

\section{ACKNOWLEDGEMENT :}

Christoph Kopper would like to thank the Theory Group at Ecole Polytechnique, especially the coauthors, for kind hospitality, and the CNRS for financial support during his stay in Palaiseau in 1991. 


\section{References}

[1 ] D.J.Gross, A.Neveu: Dynamical symmetry breaking in asymptotically free field theories, Phys. Rev.D 10(1974) 3235

[2 ] K.Gawedzki, A.Kupiainen: Gross-Neveu model through convergent perturbation expansions, Commun.Math.Phys.102(1986) 1

[3 ] J.Feldman, J.Magnen, V.Rivasseau, R.Sénéor: A Renormalizable Field Theory: The Massive Gross-Neveu Model in two Dimensions, Commun.Math.Phys.103(1986) 67

[4 ] P.A.Faria da Veiga: Construction de modèles non renormalisables en Théorie quantique des champs, thesis, Ecole Polytechnique 1991

[5 ] J.Glimm, A.Jaffe: Quantum Physics, Springer-Verlag, New York 1987

[6 ] M.C.Reed in: Constructive Field Theory, Lecture Notes in Physics 25 (1973), Proc. Erice 1973

[7 ] B.Simon: Trace Ideals and their Applications, London Mathematical Society Lecture Note Series 35, Cambridge Univ.Press (1979)

[8 ] D.Ruelle: Statistical Mechanics- Rigorous Results, Benjamin, New York (1969)

[9 ] V.Rivasseau: From Perturbative to Constructive Renormalization, Princeton University Press 1991

[10 ] D. Brydges in: Critical Phenomena, Random Systems, Gauge Theories, Proc. Les Houches 1984. North Holland (1986)

[11 ] F. Dunlop, J.Magnen, V.Rivasseau: Mass generation for an interface in the mean field regime, Ann. de l'I.H.P.58(1993) 333

[12 ] G. Battle, P. Federbush: A phase cell cluster expansion for Euclidean field theories, Ann.Phys. $142(1982) 95$

[13 ] M.C. Reed, B.Simon: Methods of Modern Mathematical Physics IV, Academic Press, New York (1979)

[14 ] E. Seiler: Schwinger functions for the Yukawa Model in two dimensions with space time cutoff, Commun.Math.Phys.42 (1975) 163

[15 ] E. Seiler, B. Simon: Bounds on the Yukawa 2 Quantum Field Theory: Upper bound on the pressure, Hamiltonian bound and linear lower bound, Commun.Math.Phys.45 (1975) 99 
[16 ] T. Balaban: Large Field Renormalization II: Localization, Exponentiation and bounds for the R-operation Commun.Math.Phys.122 (1989) 355, and references cited there. J. Magnen, V. Rivasseau, R. Sénéor: Construction of $\mathrm{YM}_{4}$ with an infrared cutoff, Commun.Math.Phys.155 (1993) 325

[17 ] P. Mitter, P. Weisz: Asymptotic scale invariance in a massive Thirring model with $U(n)$ symmetry, Phys.Rev.D8(1973) 4410

[18 ] D. Iagolnitzer, J. Magnen: Asymptotic completeness and multiparticle structure in field theories II: Theories with renormalization, Commun.Math.Phys.111(1987) 81, D. Iagolnitzer, J. Magnen: Large momentum properties and Wilson short distance expansion in non-perturbative field theory, Commun.Math.Phys.119(1988) 609

[19 ] A.B. Zamolodchikov, Al.B. Zamolodchikov: Relativistic Factorized S-Matrix in two dimensions having $\mathrm{O}(\mathrm{N})$ isotopic symmetry, Nucl.Phys.B133(1978) 525, M.Karowski, H.J.Thun: Complete S-Matrix of the $\mathrm{O}(2 \mathrm{~N})$ Gross-Neveu model, Nucl.Phys.B190(1981) 61

P. Forgacs, F. Niedermayer, P. Weisz: The exact mass gap of the Gross-Neveu model I. The thermodynamic Bethe ansatz, Nucl.Phys B367(91) 123 


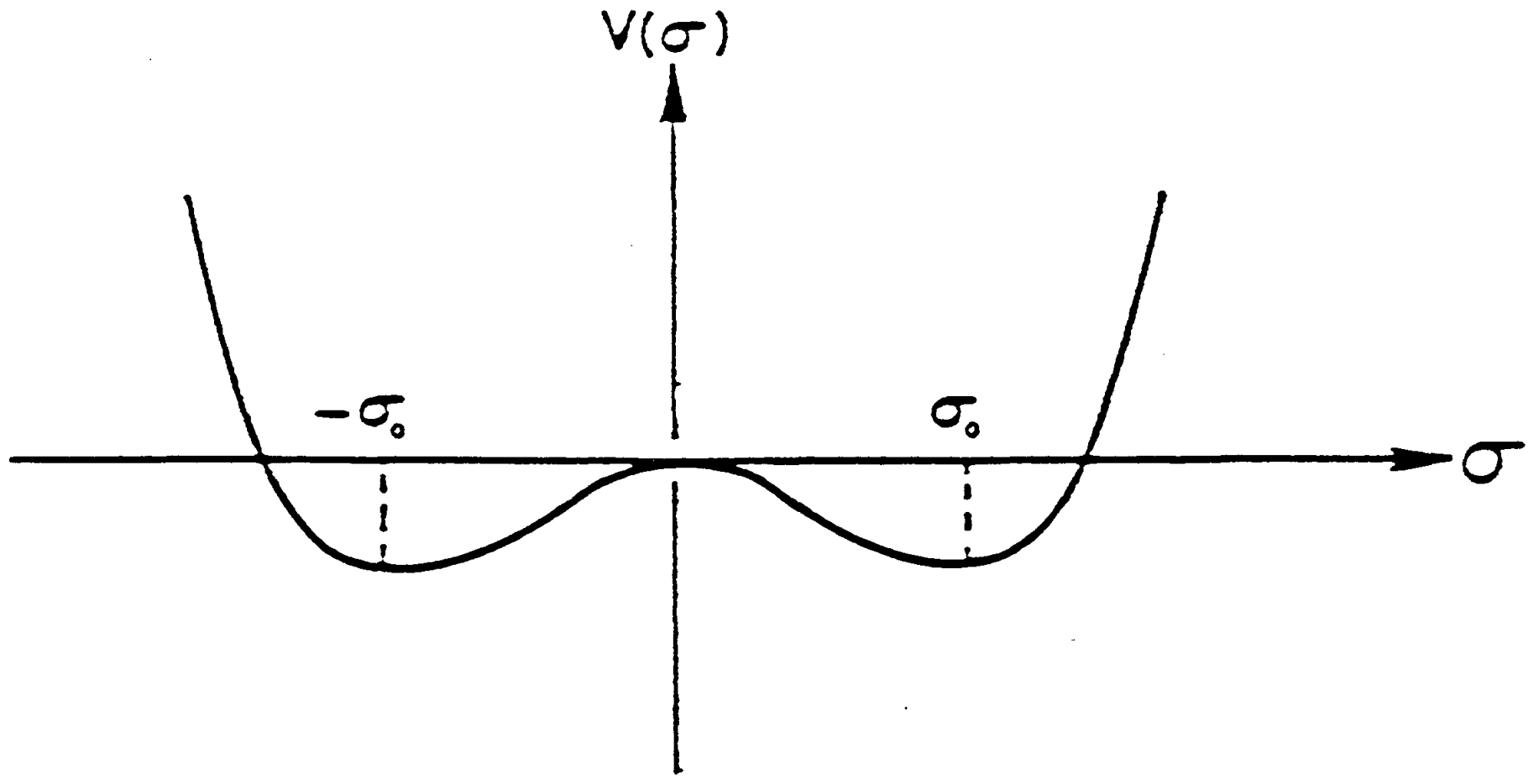

FIG.1. Form of $V(\sigma)$ to leading order in $1 / N$. 
